\title{
A few examples of local rings, II
}

\author{
Jun-ichi Nishimura
}

\begin{abstract}
This is the second part of our three-part study titled "A few examples of local rings." In the first part, we collected the basic tools and well-known important examples. But, since almost all special formal fibers of the examples there have extremely good properties, their derived normal rings are always Noetherian and analytically unramified. In this paper, we develop the basic ideas of the first part and get a somewhat improved method, which gives factorial local domains whose completion can be almost any complete local rings.
\end{abstract}

\section{Introduction}

This paper is the second part of our three-part study titled "A few examples of local rings." In the first part [8], we collected the basic tools and well-known important examples, that is, the fundamental technique of constructing local rings whose generic formal fibers are bad enough that they give examples to answer famous long-standing questions in the theory of Noetherian rings due to C. Rotthaus [12] (cf. [1]), T. Ogoma [10], [11], and R. C. Heitmann [3]. But, since almost all special formal fibers of the examples above have extremely good properties, their derived normal rings are always Noetherian and analytically unramified. On the other hand, M. Nagata has already obtained bad normal local domains, such as a two-dimensional analytically ramified factorial local domain of positive characteristic and a three-dimensional local domain of positive characteristic whose derived normal ring is not Noetherian (see [6, pp. 207-208, Examples 5, 6]; cf. [7, Examples 6.2, 6.3]).

In this intermediate part, we develop the basic ideas of the first part and get a somewhat improved method, which gives factorial local domains whose completion can be almost any complete local rings. ${ }^{\dagger}$ To be precise, we prove the following result.

\section{THEOREM 0.1}

Let $K$ be a purely transcendental extension field of countably infinite degree over a countable field $K_{0}$, let $n, r \in \mathbb{N}$, and let $z_{0}, z_{1}, \ldots, z_{n}$ be $n+1$ indeterminates over $K$. Let $R:=K\left[z_{0}, \underline{z}\right]_{\left(z_{0}, \underline{z}\right)}$, where $\underline{z}:=\left(z_{1}, \ldots, z_{n}\right)$, and let $\widehat{R}$ denote the

Kyoto Journal of Mathematics, Vol. 56, No. 1 (2016), 49-96

DOI 10.1215/21562261-3445156, (C) 2016 by Kyoto University

Received October 23, 2009. Revised October 22, 2012. Accepted December 15, 2014.

2010 Mathematics Subject Classification: Primary 13H10, 13E05, 13H05; Secondary 13C15, 13C40, 13G05.

$\dagger$ More details concerning a similar construction are given in [4, Theorem 19]. 
completion of $R$; that is, $\widehat{R}=K\left[\left[z_{0}, z\right]\right]$. Let $T_{0}:=K_{0}\left[Z_{1}, \ldots, Z_{n}\right]$ be the polynomial ring in the $n$ variables $Z_{1}, \ldots, Z_{n}$ with coefficients in $K_{0}$. For each $j$ with $1 \leq j \leq r$, let $F_{j}:=F_{j}(\underline{Z})$ be a polynomial in $T_{0}$ with zero constant term, where $\underline{Z}:=\left(Z_{1}, \ldots, Z_{n}\right)$. Suppose that (0.1.1) and (0.1.2) hold:

$$
\bigcup_{\nu \geq 1} \operatorname{Ass}\left(T_{0} /\left(F_{1}, \ldots, F_{r}\right)^{\nu}\right)=\operatorname{Ass}\left(T_{0} /\left(F_{1}, \ldots, F_{r}\right)\right) \text {. }
$$

For each $\mathfrak{P} \in \operatorname{Ass}\left(T_{0} /\left(F_{1}, \ldots, F_{r}\right)\right)$, there exists a proper subset

$$
\left\{i_{1}, \ldots, i_{t}\right\} \text { of }\{1,2, \ldots, n\} \text {, so that } \mathfrak{P} \subset \mathfrak{Q}:=\left(Z_{i_{1}}, \ldots, Z_{i_{t}}\right) T_{0} \text {. }
$$

Then there exist:

(1) elements $\zeta_{1}, \ldots, \zeta_{n} \in \widehat{R}$ that are analytically independent over $K$ such that, with $\underline{\zeta}:=\left(\zeta_{1}, \ldots, \zeta_{n}\right)$, we have $K\left[\left[z_{0}, \underline{\zeta}\right]\right]=K\left[\left[z_{0}, \underline{z}\right]\right]$, and

(2) a factorial local domain $(A, \mathfrak{m})$ with $\mathfrak{m}$-adic completion $\widehat{A}$ and a canonical inclusion map $\iota: R \hookrightarrow A$

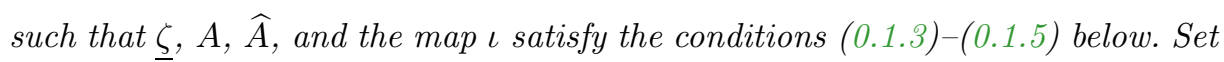

$$
f_{j}:=F_{j}(\underline{\zeta}) \in K_{0}\left[\left[z_{0}, \underline{\zeta}\right]\right] \subset K\left[\left[z_{0}, \underline{\zeta}\right]\right]=\widehat{R} .
$$

The conditions to be satisfied are as follows.

The map $\hat{\iota}: \widehat{R} \rightarrow \widehat{A}$ induced by $\iota$ is a surjection with kernel $\left(f_{1}, \ldots, f_{r}\right)$

(0.1.3) and the associated map $\tilde{\iota}: \widehat{R} /\left(f_{1}, \ldots, f_{r}\right) \hookrightarrow \widehat{A}$ is an isomorphism.

(0.1.4) $\hat{\mathfrak{q}}:=\left(\tilde{\iota}\left(\zeta_{i_{1}}\right), \ldots, \tilde{\iota}\left(\zeta_{i_{t}}\right)\right) \widehat{A}$ is a prime ideal of $\widehat{A}$ and $\hat{\mathfrak{q}} \cap A=(0)$.

(0.1.5) $\quad A / \mathfrak{p}$ is essentially of finite type over $K$, for every $\mathfrak{p} \in \operatorname{Spec}(A)$

that has height greater than one.

This theorem gives, as direct applications, the following bad local domains. (However, the detailed discussion of these examples is given in the last part of our study [9].)

\section{EXAMPLE 0.2}

We have a two-dimensional analytically ramified factorial local domain of arbitrary characteristic.

\section{EXAMPLE 0.3}

We have a two-dimensional regular local ring $(A, \mathfrak{m})$ of positive characteristic that is not Japanese but, for every height-one prime ideal $\mathfrak{p}$ of $A, A_{\mathfrak{p}}$ is a Nagata (i.e., universally Japanese) ring.

\section{EXAMPLE 0.4}

We have a three-dimensional local domain of arbitrary characteristic whose derived normal ring is not Noetherian. 


\section{EXAMPLE 0.5}

We have a three-dimensional factorial local domain whose completion has embedded associated prime ideal(s).

\section{EXAMPLE 0.6}

We have a three-dimensional factorial Nagata local domain that is not universally catenary.

Now let us summarize the contents of this paper. First we recall the well-known lemmas on regular sequences and Rees algebras in Section 1. In Sections 2 and 3, after setting the notation, we write down several elementary formulas on the relations that play essential roles later. We recommend skipping the tedious parts, namely, Sections 2.4, 2.5, 2.6, and 3.2, in the first reading and coming back to check the particular formulas when necessary.

The main body of this article consists of Sections 4 and 5. Making use of the results in the preceding sections, we prove some more technical lemmas in Section 4. The proofs of these lemmas make clear how to decompose the original prime elements of the ground regular local ring by suitably chosen prime elements. Then, we show in Section 5 that the lemmas above enable us to carry out a factorial numbering on these suitably chosen prime elements, in decomposing the original prime elements by these suitably chosen prime elements step by step. Once a good factorial numbering has been achieved, it is not a hard task to construct the desired factorial local domain, which is done in Section 6 .

In closing the Introduction, we recommend reading the beautiful original work of Nagata [6], Rotthaus [12], Ogoma [10], [11], Heitmann [3], and Brodmann and Rotthaus [1], because our study was inspired by their fundamental ideas and because, even though they are not explicitly mentioned each time, they are scattered throughout our work. We hope that our construction will be of help when new examples are wanted in the future.

Throughout this paper, all rings are commutative with 1 . A local ring $(A, \mathfrak{m})$ means a Noetherian ring $A$ with a unique maximal ideal $\mathfrak{m}$. The set of natural numbers and that of nonnegative integers are denoted, respectively, by $\mathbf{N}$ and $\mathbf{N}_{0}$. Concerning other terminology, we refer the reader to Grothendieck [2], Matsumura [5], and Nagata [6].

\section{Preliminary lemmas}

Two goals of this section are (1) to establish that $(\operatorname{Ker} \varphi) \otimes_{A} C=\operatorname{Ker}\left(\varphi \otimes \operatorname{id}_{C}\right)$ for rings $A, B, C$ and homomorphisms $\varphi: A \rightarrow B, \varphi \otimes \operatorname{id}_{C}: C \rightarrow B \otimes_{A} C$ that arise in later sections of the paper, and (2) to obtain prime ideals in rings and homomorphic images of rings that arise later. To this end, we prove 12 technical lemmas in this section. Most of the lemmas are for rings of polynomials, since the examples constructed in this paper involve rings with infinitely many indeterminates adjoined. We begin by describing conventions for dealing with indeterminates that we use in this section and extend in later sections. 


\section{CONVENTIONS 1.0}

Let $n, r \in \mathbf{N}$. We intend that, whenever $\underline{U}:=\left(U_{1}, \ldots, U_{n}\right)$ or $\underline{V}:=\left(V_{1}, \ldots, V_{r}\right)$ are adjoined to a ring $A$, they are indeterminates over $A$. We also assume the same for other elements that are adjoined, unless we indicate otherwise.

Let $Z_{0}$ and $\underline{Z}:=\left(Z_{1}, \ldots, Z_{n}\right)$ be variables over a ring $A$. Let $F_{j}:=F_{j}\left(Z_{0}, \underline{Z}\right):=$ $F_{j}\left(Z_{0}, Z_{1}, \ldots, Z_{n}\right)$ be a polynomial in the $n+1$ variables $Z_{0}, Z_{1}, \ldots, Z_{n}$ with coefficients in $A$ for each $j=1, \ldots, r$. We use the abbreviations $\underline{F}:=\underline{F}\left(Z_{0}, \underline{Z}\right):=$ $\left(F_{1}\left(Z_{0}, \underline{Z}\right), \ldots, F_{r}\left(Z_{0}, \underline{Z}\right)\right)$.

In the first lemma, we observe that the kernel commutes with the tensor product in the case of a map from a polynomial ring over a ring $A$ to a finitely generated extension of $A$ contained in $A[1 / a]$, for some non-zero divisor $a$ of $A$, and the map is tensored with a flat algebra extension $B$ of $A$.

\section{LEMMA 1.1 (CF. [3], [8, LEMMA 1.1])}

Let $S$ be a Noetherian ring, and let $q, s_{1}, \ldots, s_{n}$ be an $S$-sequence. Consider the S-homomorphism

$$
\phi: S\left[U_{1}, \ldots, U_{n}\right] \rightarrow S\left[s_{1} / q, \ldots, s_{n} / q\right] \quad \text { with } U_{i} \mapsto s_{i} / q .
$$

$P$ ut $P:=\operatorname{Ker} \phi$. Then $P$ is generated by an $S[\underline{U}]$-sequence. Namely,

$$
P=\left(q U_{1}-s_{1}, \ldots, q U_{n}-s_{n}\right) \quad \text { and } \quad P \cap S=(0) .
$$

Moreover, if $(0)=\mathfrak{q}_{1} \cap \cdots \cap \mathfrak{q}_{k}$ is a primary decomposition in $S$, then we get a canonical primary decomposition $P=\mathfrak{Q}_{1} \cap \cdots \cap \mathfrak{Q}_{k}$ in $S[\underline{U}]$. In particular, when $S$ is an integral domain, $P$ is a prime ideal of $S[\underline{U}]$.

\section{LEMMA 1.2}

With the notation of Lemma 1.1, assume in addition that $S$ is an integral domain. Then, for every $\varepsilon \in \mathbf{N}$, we see that $P:=\left(s_{1}+U_{1} q^{\varepsilon}, \ldots, s_{n}+U_{n} q^{\varepsilon}\right)$ is a prime ideal generated by the $S[\underline{U}]$-sequence $s_{1}+U_{1} q^{\varepsilon}, \ldots, s_{n}+U_{n} q^{\varepsilon}$ and that $P \cap S=(0)$.

\section{LEMMA 1.3 (CF. [8, LEMMA 1.1])}

Let $R_{0}$ be a local domain, and let $\underline{U}_{k}:=\left(U_{1 k}, \ldots, U_{n k}\right)$ be indeterminates over $R_{0}$ for $k \in \mathbf{N}$. Put

$$
R=\bigcup_{k \geq 1} R_{k}, \quad \text { where } R_{k}=R_{k-1}\left(\underline{U}_{k}\right) .
$$

Take a sequence of non-zero elements $p_{1}, p_{2}, \ldots, p_{k}, \ldots$ in $R$ such that

$$
p_{1} \in R_{0} \quad \text { and } \quad p_{k} \in R_{k-2} \quad \text { for every } k \in \mathbf{N} \text { with } k \geq 2 .
$$

\section{Put}

$$
q_{k}:=p_{1} \cdots p_{k} .
$$

Further, take $r_{1}, \ldots, r_{n} \in R_{0}$ such that $p_{1}, r_{1}, \ldots, r_{n}$ is an $R_{0}$-sequence, and let

$$
r_{i k}:=r_{i}+U_{i 1} q_{1}^{\varepsilon_{1}}+\cdots+U_{i k} q_{k}^{\varepsilon_{k}}
$$


with $\varepsilon_{j} \in \mathbf{N}$ such that $\varepsilon_{1}<\varepsilon_{2}<\cdots<\varepsilon_{k}$. Then $P_{k}:=\left(r_{1 k}, \ldots, r_{n k}\right) R$ is a prime ideal generated by an $R$-sequence $r_{1 k}, \ldots, r_{n k}$ and

$p_{h} \notin P_{k} \quad$ whenever $h \leq k+1$.

\section{LEMMA 1.4}

With the notation of Lemma 1.3, we assume in addition that $p_{1} \in R_{0}, \quad p_{k} \in R_{k-1}, \quad$ and $\quad p_{k} \notin P_{k-1} \quad$ for every $k \in \mathbf{N}$ with $k \geq 2$.

Then $P_{k}$ is a prime ideal generated by an $R$-sequence $r_{1 k}, \ldots, r_{n k}$ and $p_{h} \notin P_{k} \quad$ whenever $2 \leq h \leq k+1$.

\section{LEMMA 1.5}

Let $T$ be a ring, and let $A$ be a flat $T$-algebra with the structure homomorphism $\varphi: T \rightarrow A$. Assume that $F_{1}, \ldots, F_{r} \in T$, and assume that $\Delta$ is a non-zero divisor for T. Putting $f_{j}:=\varphi\left(F_{j}\right)$ and $q=\varphi(\Delta)$, we define ring homomorphisms, where $\underline{F} / \Delta:=\left(F_{1} / \Delta, \ldots, F_{r} / \Delta\right)$ and $f / q:=\left(f_{1} / q, \ldots, f_{r} / q\right)$ :

$$
\begin{aligned}
& \phi: T[\underline{V}] \rightarrow T[\underline{F} / \Delta] \quad \text { that maps } V_{j} \mapsto F_{j} / \Delta, \\
& \psi: A[\underline{V}] \rightarrow A[\underline{f} / q] \quad \text { that maps } V_{j} \mapsto f_{j} / q .
\end{aligned}
$$

Then $\operatorname{Ker} \psi=A \otimes \operatorname{Ker} \phi$.

Proof

We may assume $T[\underline{V}] / \operatorname{Ker} \phi \cong T[\underline{F} / \Delta] \subset T[1 / \Delta]$. Then, since $A$ is a flat $T$-algebra, the induced map $A \otimes(T[\underline{V}] / \operatorname{Ker} \phi) \rightarrow A[\underline{f} / q] \subset A[1 / q]=A \otimes T[1 / \Delta]$ is injective and this gives the assertion.

\section{LEMMA 1.6}

Let $T:=L[\Delta, \underline{Z}]$ be a polynomial ring in $n+1$ variables over a field $L$, and let $A$ be a flat $T$-algebra with the structure homomorphism $\varphi: T \rightarrow A$ that maps $\Delta$ to $q$ and $Z_{i}$ to $z_{i}$. Taking $F_{1}(\Delta, \underline{Z}), \ldots, F_{r}(\Delta, \underline{Z}) \in T$, we define ring homomorphisms:

$$
\begin{aligned}
& \phi: T[\underline{V}] \rightarrow T[\underline{F} / \Delta] \text { that maps } V_{j} \mapsto F_{j}(\Delta, \underline{Z}) / \Delta, \\
& \psi: A[\underline{V}] \rightarrow A[\underline{F} / q] \quad \text { that maps } V_{j} \mapsto F_{j}(q, \underline{z}) / q,
\end{aligned}
$$

where $\underline{z}:=\left(z_{1}, \ldots, z_{n}\right)$ and $\underline{F} / q:=\left(F_{1}(q, \underline{z}) / q, \ldots, F_{r}(q, \underline{z}) / q\right)$. Assume that $\operatorname{Ker} \phi=\left(G_{1}(\Delta, \underline{Z}, \underline{V}), \ldots, G_{s}(\Delta, \underline{Z}, \underline{V})\right)$. Then

$$
\operatorname{Ker} \psi=\left(G_{1}(q, \underline{z}, \underline{V}), \ldots, G_{s}(q, \underline{z}, \underline{V})\right) .
$$

\section{LEMMA 1.7}

With $T$ of Lemma 1.6, assume that $F_{1}, \ldots, F_{r} \in T_{0}=L[\underline{Z}] \subset T$. Let $\phi: T[\underline{V}] \rightarrow$ $T[\underline{F} / \Delta]$ be a T-homomorphism that maps $V_{j}$ to $F_{j}(\underline{Z}) / \Delta$. Then

$$
\operatorname{Ker} \phi=\left(\Delta V_{1}-F_{1}(\underline{Z}), \ldots, \Delta V_{r}-F_{r}(\underline{Z}), G_{1}(\underline{Z}, \underline{V}), \ldots, G_{s}(\underline{Z}, \underline{V})\right),
$$

where $G_{h}(\underline{Z}, \underline{V}) \in L[\underline{Z}, \underline{V}]$ and they are homogeneous in $\underline{V}$. 


\section{Proof}

Take $H(\Delta, \underline{Z}, \underline{V}) \in \operatorname{Ker} \phi$. We may assume that $H$ contains no monomials divided by $\Delta V_{j}$. That is,

$$
H(\Delta, \underline{Z}, \underline{V}):=H_{1}(\Delta, \underline{Z})+H_{2}(\underline{Z}, \underline{V}),
$$

where $H_{1} \in L[\Delta, \underline{Z}]$ and $H_{2} \in L[\underline{Z}, \underline{V}]$. Then, replacing $V_{j}$ by $F_{j}(\underline{Z}) / \Delta$, we have

$$
H(\Delta, \underline{Z}, \underline{F}(\underline{Z}) / \Delta)=H_{1}(\Delta, \underline{Z})+H_{2}(\underline{Z}, \underline{F} / \Delta)=0
$$

in $L[\Delta, \underline{Z}, 1 / \Delta]$. Regarding $H(\Delta, \underline{Z}, \underline{F} / \Delta)$ as a Laurent polynomial in $\Delta$, we see that each homogeneous part of $H$ with respect to $\underline{V}$ belongs to $\operatorname{Ker} \phi$.

\section{LEMMA 1.8}

With the notation above, let ${ }^{a} \phi: \operatorname{Spec}(T[\underline{F} / \Delta]) \rightarrow \operatorname{Spec}(T)$ be the canonical map. Then

$$
{ }^{a} \phi(\operatorname{Ass}(T[\underline{F} / \Delta] / \Delta T[\underline{F} / \Delta]))=\bigcup_{\nu \geq 1} \operatorname{Ass}\left(T /\left(\Delta,(\underline{F})^{\nu}\right)\right) .
$$

Proof

By our construction, we have the following canonical isomorphism of $T$-algebras:

$$
T[\underline{F} / \Delta] / \Delta T[\underline{F} / \Delta] \cong \bigoplus_{\nu \geq 0}(\underline{\bar{F}})^{\nu} \bar{T} /(\underline{\bar{F}})^{\nu+1} \bar{T},
$$

where $\bar{T}:=T / \Delta T$ and $\underline{\bar{F}}:=\left(\bar{F}_{1}(\Delta, \underline{Z}), \ldots, \bar{F}_{r}(\Delta, \underline{Z})\right)=\left(\bar{F}_{1}(0, \underline{Z}), \ldots, \bar{F}_{r}(0, \underline{Z})\right)$. Then the assertion follows from the definition of Ass (i.e., the set of associated prime ideals).

\section{LEMMA 1.9}

Let $(A, \mathfrak{m})$ be a local ring, let $\underline{a}:=\left(a_{1}, \ldots, a_{r}\right)$ be elements of $A$, and let $q$ be a non-zero divisor of $A$. Putting $\alpha_{j}:=a_{j} / q \in Q(A)$, the total quotient ring of $A$, let $\underline{\alpha}:=\left(\alpha_{1}, \ldots, \alpha_{r}\right)$, and we consider the surjective A-homomorphisms:

$$
\begin{gathered}
\phi: A[\underline{V}] \rightarrow A[\underline{\alpha}] \quad \text { that maps } V_{j} \mapsto \alpha_{j}, \\
\phi_{U}: A[\underline{V}]_{U} \rightarrow A[\underline{\alpha}]_{\phi(U)} \quad \text { with } U=A[\underline{V}] \backslash(\mathfrak{m}, \underline{V}) .
\end{gathered}
$$

Taking an element $b \in \mathfrak{m}$, we put $\bar{A}=A / b A$ and $\overline{\mathfrak{m}}=\mathfrak{m} / b A$. Let $\bar{q}, \bar{a}_{1}, \ldots, \bar{a}_{r}$, respectively, be the images of $q, a_{1}, \ldots, a_{r}$ in $\bar{A}$. When $\bar{q}$ is a non-zero divisor, putting $\bar{\alpha}_{j}:=\bar{a}_{j} / \bar{q} \in Q(\bar{A})$, let $\underline{\bar{\alpha}}:=\left(\bar{\alpha}_{1}, \ldots, \bar{\alpha}_{r}\right)$, and we can consider the induced surjective $\bar{A}$-homomorphisms:

$$
\begin{aligned}
& \bar{\phi}: \bar{A}[\underline{V}] \rightarrow \bar{A}[\underline{\bar{\alpha}}] \quad \text { that maps } V_{j} \mapsto \bar{\alpha}_{j}, \\
& \bar{\phi}_{\bar{U}}: \bar{A}[\underline{V}]_{\bar{U}} \rightarrow \bar{A}[\underline{\bar{\alpha}}]_{\bar{\phi}(\bar{U})} \text { with } \bar{U}=\bar{A}[\underline{V}] \backslash(\overline{\mathfrak{m}}, \underline{V}) .
\end{aligned}
$$

Assume that $b \notin \operatorname{Ass}\left(A[\underline{\alpha}]_{\phi(U)} / q A[\underline{\alpha}]_{\phi(U)}\right)$. Then

$$
\bar{A}[\underline{\bar{\alpha}}]_{\bar{\phi}(\bar{U})} \cong \bar{A} \otimes A[\underline{\alpha}]_{\phi(U)} .
$$

That is, $\operatorname{Ker} \bar{\phi}_{\bar{U}}=\bar{A} \otimes \operatorname{Ker} \phi_{U}$. In particular, if $b$ is a prime element of $A$, then $b$ is a prime element of $A[\underline{\alpha}]_{\phi(U)}$. 
Proof

On the one hand, by the following diagram whose rows are exact

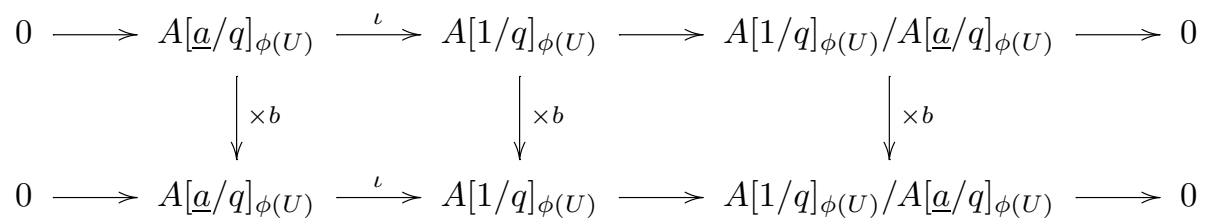

where $\underline{a} / q:=\left(a_{1} / q, \ldots, a_{r} / q\right)=\left(\alpha_{1}, \ldots, \alpha_{r}\right)=\underline{\alpha}$, we get an exact sequence

$$
\bar{A} \otimes A[a / q]_{\phi(U)} \stackrel{\bar{\iota}}{\longrightarrow} \bar{A}[1 / \bar{q}]_{\bar{\phi}(\bar{U})} \longrightarrow \bar{A}[1 / \bar{q}]_{\bar{\phi}(\bar{U})} / \bar{A}[\bar{a} / \bar{q}]_{\bar{\phi}(\bar{U})} \longrightarrow 0 .
$$

On the other hand, we have a commutative diagram with exact diagonal maps

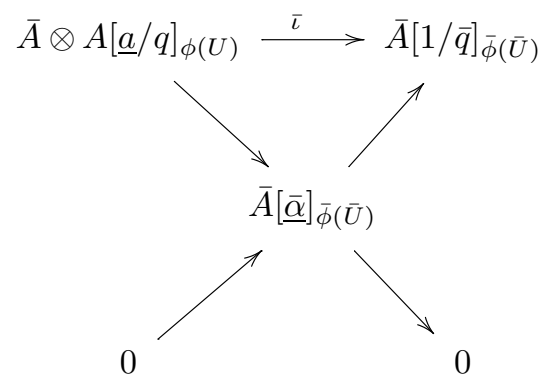

where $\bar{\iota}$ is injective by assumption. Thus, $\bar{A} \otimes \operatorname{Ker} \phi_{U}=\operatorname{Ker} \bar{\phi}_{\bar{U}}$.

\section{LEMMA 1.10}

Let $A$ be a Noetherian ring with $q, a_{1}, \ldots, a_{r} \in A$, where we assume that $q$ is a non-zero divisor for $A$. Putting $\alpha_{j}:=a_{j} / q \in Q(A)$, we set $\tilde{\mathfrak{a}}:=\left(\alpha_{1}, \ldots, \alpha_{r}\right) A[\underline{\alpha}]$, where $\underline{\alpha}:=\left(\alpha_{1}, \ldots, \alpha_{r}\right)$. Take an ideal $\mathfrak{b}:=\left(b_{1}, \ldots, b_{n}\right)$ of $A$, and denote by $\bar{q}$, $\bar{a}_{j}$, respectively, the images of $q, a_{j}$ in $\bar{A}:=A / \mathfrak{b}$, where we further assume that $\bar{q}$ is a non-zero divisor for $\bar{A}$. Putting $\bar{\alpha}_{j}:=\bar{a}_{j} / \bar{q} \in Q(\bar{A})$, we consider an $\bar{A}$ homomorphism $\bar{\phi}: \bar{A}[\underline{V}] \rightarrow \bar{A}[\underline{\bar{\alpha}}]$ that maps $V_{j} \mapsto \bar{\alpha}_{j}$. Suppose that

$$
\operatorname{Ker} \bar{\phi}=\left(\bar{q} V_{1}-\bar{a}_{1}, \ldots, \bar{q} V_{r}-\bar{a}_{r}, \bar{G}_{1}(\underline{V}), \ldots, \bar{G}_{s}(\underline{V})\right) .
$$

Then $\tilde{\mathfrak{b}}:=\mathfrak{b} A[1 / q] \cap A[\underline{\alpha}]=\left(b_{1}, \ldots, b_{n}, G_{1}(\underline{\alpha}), \ldots, G_{s}(\underline{\alpha})\right)$, where $G_{h}(\underline{V}) \in A[\underline{V}]$ is a preimage of $\bar{G}_{h}(\underline{V})$. Assume further that $G_{h}(\underline{V}) \in(\underline{V}) A[\underline{V}]$ for $h=1, \ldots, s$. Then

$$
\tilde{\mathfrak{a}} \cap \tilde{\mathfrak{b}}=\left(b_{1} \cdot \alpha_{1}, \ldots, b_{n} \cdot \alpha_{r}, G_{1}(\underline{\alpha}), \ldots, G_{s}(\underline{\alpha})\right) .
$$

LEMMA 1.11

Let $A$ be a local ring that contains a field $L$, and let $a_{1}, \ldots, a_{n}$ be an $A$-sequence. Take polynomials without constant terms $F_{1}(\underline{Z}), \ldots, F_{r}(\underline{Z}) \in(\underline{Z}) L[\underline{Z}]$, and let $I:=\left(F_{1}(\underline{a}), \ldots, F_{r}(\underline{a})\right)$, where $\underline{a}:=\left(a_{1}, \ldots, a_{n}\right)$. Assume that the residue field of $A$ is separable over $L$. Then, for each $\mathfrak{p} \in \operatorname{Ass}(A)$, there exists at least one $P \in$ $\operatorname{Ass}(A / I)$ such that $P \supset \mathfrak{p}$. 


\section{Proof}

Let $T:=L[\underline{Z}]_{(Z)}$, a regular local ring, and let $\varphi: T \rightarrow A$ be a local $L$-homomorphism that maps $Z_{i} \mapsto a_{i}$. Because $a_{1}, \ldots, a_{n}$ is an $A$-sequence, $\varphi$ makes $A$ a flat $T$-algebra. We may assume that $A$ is complete. Thus, there exists a canonical projection $\pi: R \rightarrow A \cong R / \operatorname{Ker} \pi$, where $R$ is a regular local ring with $L \subseteq R$. For each $i$, choose $z_{i} \in R$ with $\pi\left(z_{i}\right)=a_{i}$. Then $\varphi$ lifts to $R$; that is, we have a local $L$-homomorphism $\Phi: T \rightarrow R$ that maps $Z_{i} \mapsto z_{i}$ such that $\varphi=\pi \circ \Phi$. Because $z_{1}, \ldots, z_{n}$ forms an $R$-sequence by [6, (42.8)], $\Phi$ makes $R$ a flat $T$-algebra too.

Let $\mathfrak{p} \in \operatorname{Ass}(A)$. Compare the heights of $J:=\left(F_{1}(\underline{Z}), \ldots, F_{r}(\underline{Z})\right), I_{0}:=\left(F_{1}(\underline{z})\right.$, $\left.\ldots, F_{r}(\underline{z})\right)$, where $\underline{z}:=\left(z_{1}, \ldots, z_{n}\right)$, and $\bar{I}=(I+\mathfrak{p}) / \mathfrak{p}=\left(I_{0}+\mathfrak{P}\right) / \mathfrak{P}$, where $\mathfrak{P}$ is the prime ideal of $R$ that corresponds to $\mathfrak{p}$. Suppose that ht $J=h$. Then ht $I_{0}=h$. Hence, ht $\bar{I} \leq h$ by $[6,(42.8)]$. Take a prime ideal $P$ of $A$ that contains $I+\mathfrak{p}$ and that satisfies the inequality $\operatorname{ht}(P / \mathfrak{p}) \leq h$. Then $\operatorname{depth} A_{P} \leq h$ by [5, (15.E)]. Consequently, depth $T_{Q} \leq h$ for $Q=P \cap T$. Thus, $Q$ contains $J$ and ht $Q \leq h$. This means that $Q \in \operatorname{Ass}(T / J)$ and that ht $Q=\operatorname{depth} T_{Q}=h$. Therefore, $P \in \operatorname{Ass}(A / I)$.

\section{LEMMA 1.12}

Let $A$ be a ring. Take ideals $I, J$ of $A$, and take $a, b \in A$. Assume that $a$ is a non-zero divisor for $A /(I+J)$, and assume that $b$ is a non-zero divisor for $A /(a A+I+J)$. Then

$$
(a A+I) \cap(b A+J)=a b A+a J+b I+I \cap J .
$$

\section{Proof}

It suffices to show that the left-hand side of the displayed equation is contained in the right-hand side. Take $x \in(a A+I) \cap(b A+J)$. Then $x=a y+\alpha=b z+\beta$ with $y, z \in A, \alpha \in I$, and $\beta \in J$. Thus, $z \in((a A+I+J): b A)=(a A+I+J)$; that is, $z=a w+\gamma+\delta$, where $w \in A, \gamma \in I$, and $\delta \in J$. Hence,

$$
x=b(a w+\gamma+\delta)+\beta=a b w+b \gamma+(b \delta+\beta) \in a b A+b I+J .
$$

Therefore, $b \delta+\beta \in J \cap(a A+I)$. We claim that $J \cap(a A+I)=a J+I \cap J$.

Proof of the claim. Take $\xi \in J \cap(a A+I)$, and write $\xi=a u+\omega$ with $u \in A$ and $\omega \in I$. Then $u \in((I+J): a A)=I+J$; that is, $u=\tau+\sigma$ with $\tau \in I$ and $\sigma \in J$. Thus, $\xi=a(\tau+\sigma)+\omega=a \sigma+(a \tau+\omega) \in a J+I \cap J$.

\section{Primary relations}

\subsection{Notation and terminology}

Let $K_{0}$ be a countable field. Let $K$ be a purely transcendental extension field of countable degree over $K_{0}$, say,

$$
K:=\bigcup_{k \in \mathbf{N}} K_{0}\left(\left\{a_{i \ell}, b_{i \ell}, c_{j \ell} \mid i=1, \ldots, n ; j=1, \ldots, r ; \ell=1, \ldots, k\right\}\right),
$$

where $n, r \in \mathbf{N}$ and $\left\{a_{i \ell}, b_{i \ell}, c_{j \ell} \mid i=1, \ldots, n ; j=1, \ldots, r ; \ell=1, \ldots, k\right\}$ is a transcendental basis over $K_{0}$. Let $\underline{a_{k}}:=\left(a_{1 k}, \ldots, a_{n k}\right)$, let $\underline{b_{k}}:=\left(b_{1 k}, \ldots, b_{n k}\right)$, let 
$\underline{c_{k}}:=\left(c_{1 k}, \ldots, c_{r k}\right)$, let $\underline{a}:=\left(\left\{\underline{a_{k}}\right\}_{k \in \mathbf{N}}\right)$, let $\underline{b}:=\left(\left\{\underline{b_{k}}\right\}_{k \in \mathbf{N}}\right)$, and let $\underline{c}:=\left(\left\{\underline{c_{k}}\right\}_{k \in \mathbf{N}}\right)$. Let $K_{k}:=K_{k-1}\left(\underline{a_{k}}, \underline{b_{k}}, \underline{c_{k}}\right)$ for every $k \in \mathbf{N}$. Then

$$
K=\bigcup_{k \in \mathbf{N}} K_{k}=K_{0}(\underline{a}, \underline{b}, \underline{c}) .
$$

Let $\underline{z}:=\left(z_{1}, \ldots, z_{n}\right)$ and $\underline{w}:=\left(w_{1}, \ldots, w_{r}\right)$ be indeterminates over $K$. Let $S_{0}:=$ $K_{0}\left[z_{0}, \underline{z}, \underline{w}\right]$ be a polynomial ring with prime ideal $\mathfrak{N}_{0}:=\left(z_{0}, \underline{z}, \underline{w}\right) S_{0}$, and let $R_{0}:=\left(S_{0}\right)_{\mathfrak{N}_{0}}=K_{0}\left[z_{0}, \underline{z}, \underline{w}\right]_{\left(z_{0}, \underline{z}, \underline{w}\right)}$. Then

(2.1.0) $\quad R_{0}$ is a regular local ring with maximal ideal $\mathfrak{n}_{0}:=\left(z_{0}, \underline{z}, \underline{w}\right) R_{0}$.

Let $S_{k}:=S_{k-1}\left[\underline{a_{k}}, \underline{b_{k}}, \underline{c_{k}}\right]$ be a polynomial ring with prime ideal $\mathfrak{N}_{k}:=\left(z_{0}, \underline{z}, \underline{w}\right) S_{k}$, and let $R_{k}:=\left(\overline{S_{k}}\right)_{\mathfrak{N}_{k}}=K_{k}\left[z_{0}, \underline{z}, \underline{w}\right]_{\left(z_{0}, \underline{z}, \underline{w}\right)}$. Then

$$
R_{k} \text { is a regular local ring with maximal ideal } \mathfrak{n}_{k}:=\left(z_{0}, \underline{z}, \underline{w}\right) R_{k} .
$$

Further, put

$$
S:=\bigcup_{k \in \mathbf{N}} S_{k}=K_{0}\left[\underline{a}, \underline{b}, \underline{c}, z_{0}, \underline{z}, \underline{w}\right] \text { with prime ideal } \mathfrak{N}:=\left(z_{0}, \underline{z}, \underline{w}\right) S,
$$

and let $R:=S_{\mathfrak{N}}=K\left[z_{0}, \underline{z}, \underline{w}\right]_{\left(z_{0}, \underline{z}, \underline{w}\right)}$. Then

(2.1.3) $R$ is a countable regular local ring with maximal ideal $\mathfrak{n}:=\left(z_{0}, \underline{z}, \underline{w}\right) R$ such that

$$
R_{k}=R_{k-1}\left(\underline{a_{k}}, \underline{b_{k}}, \underline{c_{k}}\right) \quad \text { and } \quad R=\bigcup_{k \in \mathbf{N}} R_{k}
$$

\subsection{Polynomial relations}

With the notation of Section 2.1, we consider $r$ polynomials in $n$ variables over $K_{0}$ with zero constant term: $F_{1}(\underline{Z}), \ldots, F_{r}(\underline{Z}) \in(\underline{Z}) K_{0}[\underline{Z}]$.

Let $\left\{\Delta_{i g}, \nabla_{i h} \mid 1 \leq i \leq n\right.$ and $\left.g, h \in \mathbf{N}_{0}\right\}$ be a countably infinite set of indeterminates over $K_{0}[\underline{Z}]$. Define, for each $\ell, m \in \mathbf{N}$,

$$
X_{i \ell}:=\sum_{g=0}^{\ell} \Delta_{i g}, \quad Y_{i m}:=\sum_{h=0}^{m} \nabla_{i h}, \quad \text { and } \quad Z_{i \ell m}:=X_{i \ell}+Y_{i m} .
$$

Given a monomial in the $n(\ell+1)$ indeterminates $\Delta_{i g}$, where $1 \leq i \leq n$ and $0 \leq$ $g \leq \ell$, we abbreviate the monomial by $\Delta^{E^{+}(\ell)}$, where $E^{+}(\ell):=\left(e_{10}^{+}, \ldots, e_{n \ell}^{+}\right) \in$ $\mathbf{N}_{0}^{n(\ell+1)}$ is the ordered tuple of $n(\ell+1)$ exponents that occur in the monomial. That is,

$$
\Delta^{E^{+}(\ell)}:=\Delta_{10}^{e_{10}^{+}} \cdots \Delta_{n \ell}^{e_{n \ell}^{+}} .
$$

Similarly for monomials in the $n(m+1)$ indeterminates $\nabla_{i h}$, where $1 \leq i \leq n$ and $0 \leq h \leq m$, set

$$
\nabla^{E^{-}(m)}:=\nabla_{10}^{e_{10}^{-}} \cdots \nabla_{n m}^{e_{n m}^{-}},
$$

where $E^{-}(m):=\left(e_{10}^{-}, \ldots, e_{n m}^{-}\right) \in \mathbf{N}_{0}^{n(m+1)}$. Note that in [9] we continue to use + to designate expressions involving or dominated by the indeterminates $\Delta_{i g}$, and 
we use - to designate expressions involving or dominated by the indeterminates $\nabla_{i h}$. Let

$$
\begin{aligned}
\underline{\Delta_{\ell}} & :=\left(\Delta_{10}, \Delta_{11}, \ldots, \Delta_{1 \ell}, \Delta_{20}, \ldots, \Delta_{n \ell}\right), \\
\underline{\nabla_{m}} & :=\left(\nabla_{10}, \nabla_{11}, \ldots, \nabla_{1 m}, \nabla_{20}, \ldots, \nabla_{n m}\right),
\end{aligned}
$$

and let $Z_{\ell m}:=\left(Z_{1 \ell m}, \ldots, Z_{n \ell m}\right)$. Then, for each $j$ with $1 \leq j \leq r$, we write $F_{j}\left(\underline{Z_{\ell m}}\right)$ in terms of monomials in the $\Delta_{i g}$ 's and $\nabla_{i h}$ 's as follows:

$$
\begin{aligned}
F_{j}\left(\underline{Z_{\ell m}}\right) & :=F_{j}\left(\sum_{g=0}^{\ell} \Delta_{1 g}+\sum_{h=0}^{m} \nabla_{1 h}, \ldots, \sum_{g=0}^{\ell} \Delta_{n g}+\sum_{h=0}^{m} \nabla_{n h}\right) \\
& =F_{j}\left(\Delta_{10}+\cdots+\Delta_{1 \ell}+\nabla_{10}+\cdots+\nabla_{1 m}, \ldots, \Delta_{n 0}+\cdots+\nabla_{n m}\right) \\
& =\sum k_{j E^{+}(\ell) E^{-}(m)} \Delta^{E^{+}(\ell)} \nabla^{E^{-}(m)} \in K_{0}\left[\underline{\Delta_{\ell}}, \underline{\nabla_{m}}\right],
\end{aligned}
$$

for some elements $k_{j E^{+}(\ell) E^{-}(m)} \in K_{0}$, where the sum is taken over every monomial $\Delta^{E^{+}(\ell)} \nabla^{E^{-}(m)}$ that occurs in $F_{j}\left(\underline{Z_{\ell m}}\right)$. With the terminology above, we define, for each $E^{+}(\ell)$ and $E^{-}(m)$,

$$
\begin{gathered}
o\left(E^{+}(\ell)\right):=\max \left\{0, g \mid 0 \leq g \leq \ell \text { and } e_{i g}^{+}>0 \text { for some } i \text { with } 1 \leq i \leq n\right\}, \\
o\left(E^{-}(m)\right):=\max \left\{0, h \mid 0 \leq h \leq m \text { and } e_{i h}^{-}>0 \text { for some } i \text { with } 1 \leq i \leq n\right\} .
\end{gathered}
$$

That is, $o\left(E^{+}(\ell)\right)$ is the largest $g \leq \ell$ such that the exponent of $\Delta_{i g}$ is positive in $\Delta^{E^{+}(\ell)}$ for some $i$, and similarly for $o\left(E^{-}(m)\right)$. Next we write

$$
F_{j}\left(\underline{Z_{\ell m}}\right)=F_{j}^{+}\left(\underline{Z_{\ell m}}\right)+F_{j}^{-}\left(\underline{Z_{\ell m}}\right) \text {, }
$$

where

$$
F_{j}^{+}\left(\underline{Z_{\ell m}}\right):=\sum_{o\left(E^{+}(\ell)\right)>o\left(E^{-}(m)\right)} k_{j E^{+}(\ell) E^{-}(m)} \Delta^{E^{+}(\ell)} \nabla^{E^{-}(m)}
$$

and

$$
F_{j}^{-}\left(\underline{Z_{\ell m}}\right):=\sum_{o\left(E^{+}(\ell)\right) \leq o\left(E^{-}(m)\right)} k_{j E^{+}(\ell) E^{-}(m)} \Delta^{E^{+}(\ell)} \nabla^{E^{-}(m)} .
$$

Let $B$ be a ring, and let $\underline{Z}:=Z_{1}, \ldots, Z_{n}$ be variables over $B$. Take $F(\underline{Z}) \in$ $B[\underline{Z}]$, and take variables $\underline{\Omega}:=\left(\Omega_{1}, \ldots, \Omega_{n}\right)$ over $B[\underline{Z}]$. By Taylor's expansion, we get

$$
F(\underline{Z}+\underline{\Omega})=F(\underline{Z})+\sum \frac{\partial^{D} F}{(\partial Z)^{D}}(\underline{Z}) \Omega^{D} \in B[\underline{Z}, \underline{\Omega}],
$$

where $\underline{Z}+\underline{\Omega}:=\left(Z_{1}+\Omega_{1}, \ldots, Z_{n}+\Omega_{n}\right)$,

$$
\frac{\partial^{D} F}{(\partial Z)^{D}}(\underline{Z}):=\frac{1}{d_{1} ! \cdots d_{n} !} \cdot \frac{\partial^{d_{1}+\cdots+d_{n}} F}{\left(\partial Z_{1}\right)^{d_{1}} \cdots\left(\partial Z_{n}\right)^{d_{n}}}(\underline{Z}),
$$


and $\Omega^{D}:=\Omega_{1}^{d_{1}} \cdots \Omega_{n}^{d_{n}}$, and the sum is taken over all $D:=\left(d_{1}, \ldots, d_{n}\right) \in \mathbf{N}_{0}^{n} \backslash$ $\{(0, \ldots, 0)\}$. Note that the formula above works in arbitrary characteristic. Hence, with the notation above, we have

$$
\begin{aligned}
F_{j}\left(\underline{Z_{(k+1) k}}\right) & :=F_{j}\left(Z_{1 k k}+\Delta_{1(k+1)}, \ldots, Z_{n k k}+\Delta_{n(k+1)}\right) \\
& =F_{j}\left(\underline{Z_{k k}}\right)+\sum \frac{\partial^{D} F_{j}}{(\partial Z)^{D}}\left(\underline{Z_{k k}}\right) \Delta_{k+1}^{D} .
\end{aligned}
$$

Thus,

$$
\begin{aligned}
& F_{j}^{+} \underline{\left(Z_{(k+1) k}\right)}=F_{j}^{+}\left(\underline{Z_{k k}}\right)+\sum \frac{\partial^{D} F_{j}}{(\partial Z)^{D}}\left(\underline{Z_{k k}}\right) \Delta_{k+1}^{D}, \\
& F_{j}^{-} \underline{\left(\underline{Z_{(k+1) k}}\right)}=F_{j}^{-}\left(\underline{Z_{k k}}\right) .
\end{aligned}
$$

By the same reasoning, we have

$$
\begin{aligned}
& F_{j}^{+}\left(\underline{Z_{(k+1)(k+1)}}\right)=F_{j}^{+}\left(\underline{Z_{(k+1) k}}\right), \\
& F_{j}^{-}\left(\underline{Z_{(k+1)(k+1)}}\right)=F_{j}^{-}\left(\underline{Z_{(k+1) k}}\right)+\sum \frac{\partial^{D} F_{j}}{(\partial Z)^{D}}\left(\underline{Z_{(k+1) k}}\right) \nabla_{k+1}^{D} .
\end{aligned}
$$

\subsection{Numbering, I}

With the notation as in Section 2.1, suppose that $\underline{x}:=\left(x_{1}, \ldots, x_{n}\right)$ and $\underline{y}:=$ $\left(y_{1}, \ldots, y_{n}\right)$ are elements of $R_{0}$ that satisfy the following:

$$
z_{i}=x_{i}+y_{i} \quad \text { for } i=1, \ldots, n,
$$

(2.3.1) $z_{0}, x_{1}, \ldots, x_{n}, w_{1}, \ldots, w_{r}$ form regular systems of parameters of $R$,

(2.3.2) $z_{0}, y_{1}, \ldots, y_{n}, w_{1}, \ldots, w_{r}$ form regular systems of parameters of $R$.

Inductively, we define two sequences of elements of the field of quotients $Q(R)$

$$
\pi_{1}, \pi_{2}, \ldots, \pi_{k}, \ldots \quad \text { and } \quad p_{1}, p_{2}, \ldots, p_{k}, \ldots
$$

in the following manner (for the details, see Section 5):

$$
\pi_{1}:=z_{0} \quad \text { and } \quad p_{1} \in R_{0} .
$$

Supposing that $\pi_{1}, \ldots, \pi_{k}$ and $p_{1}, \ldots, p_{k}$ are already chosen, we write

$$
q_{\ell}:=p_{1} \cdots p_{\ell} \quad \text { and } \quad \theta_{m}:=\pi_{1} \cdots \pi_{m} \quad \text { for } \ell, m \leq k .
$$

By choosing suitable natural numbers $\varepsilon_{1}, \ldots, \varepsilon_{k}$, we set

$$
t_{h}:=q_{h}^{\varepsilon_{h}} \theta_{h} \quad \text { for } h \leq k .
$$

For $h, \ell, m \leq k$, we put

$$
\begin{aligned}
x_{i \ell} & :=x_{i}+a_{i 1} q_{1}^{\varepsilon_{1}}+\cdots+a_{i \ell} q_{\ell}^{\varepsilon_{\ell}}, \\
\eta_{i m} & :=y_{i}+b_{i 1} \theta_{1}^{\varepsilon_{1}}+\cdots+b_{i m} \theta_{m}^{\varepsilon_{m}}, \\
w_{j h} & :=w_{j}+c_{j 1} t_{1}^{\varepsilon_{1}}+\cdots+c_{j h} t_{h}^{\varepsilon_{h}}, \\
\zeta_{i \ell m} & :=x_{i \ell}+\eta_{i m} .
\end{aligned}
$$


With the notation above, put

$$
\begin{aligned}
& \phi_{j \ell m}^{+}:=F_{j}^{+}\left(\underline{\zeta_{\ell m}}\right), \\
& \phi_{j \ell m}^{-}:=F_{j}^{-}\left(\underline{\zeta_{\ell m}}\right) .
\end{aligned}
$$

Here we use the abbreviations as in Conventions 1.0, namely, $\underline{x_{\ell}}:=\left(x_{1 \ell}, \ldots, x_{n \ell}\right)$, $\underline{\eta_{m}}:=\left(\eta_{1 m}, \ldots, \eta_{n m}\right), \underline{w_{h}}:=\left(w_{1 h}, \ldots, w_{r h}\right)$, and $\underline{\zeta_{\ell m}}:=\left(\zeta_{1 \ell m}, \ldots, \zeta_{n \ell m}\right)$. Further, $\overline{\text { for }}$ suitable natural numbers $\nu_{1}, \ldots, \nu_{k}$, let

$$
\begin{aligned}
& \alpha_{j \ell m}^{+}:=\frac{1}{q_{\ell}^{\nu_{\ell}}}\left(\phi_{j \ell m}^{+}+w_{j \ell}\right), \\
& \alpha_{j \ell m}^{-}:=\frac{1}{\theta_{m}^{\nu_{m}}}\left(\phi_{j \ell m}^{-}-w_{j m}\right) .
\end{aligned}
$$

Finally, take $\pi_{k+1}, p_{k+1}$ that satisfy

$$
\begin{aligned}
& p_{k+1} \in R_{k-1}, \\
& \pi_{k+1}:=r_{k+1}+\sum r_{(k+1) H}\left(q_{k} \alpha_{k k}^{+}\right)^{H},
\end{aligned}
$$

where $\left(q_{k} \alpha_{k k}^{+}\right)^{H}:=\left(q_{k} \alpha_{1 k k}^{+}\right)^{h_{1}} \cdots\left(q_{k} \alpha_{r k k}^{+}\right)^{h_{r}}$ and $r_{k+1}, r_{(k+1) H} \in R_{k}$, and the sum is taken over all $H:=\left(h_{1}, \ldots, h_{r}\right) \in \mathbf{N}_{0}^{r} \backslash\{(0, \ldots, 0)\}$. Thus, by repeating the process above, we get two sequences

$$
\pi_{1}, \pi_{2}, \ldots, \pi_{k}, \ldots \quad \text { and } \quad p_{1}, p_{2}, \ldots, p_{k}, \ldots
$$

Here we remark that, when the $\varepsilon_{k}$ 's are chosen sufficiently large,

$$
\begin{aligned}
& q_{k}:=p_{1} \cdots p_{k} \in R_{k-2}, \\
& \theta_{k}:=\pi_{1} \cdots \pi_{k} \in R_{k-1}\left[q_{k-1} \underline{\alpha_{(k-1)(k-1)}^{+}}\right],
\end{aligned}
$$

where $q_{k-1} \underline{\alpha_{(k-1)(k-1)}^{+}}:=\left(q_{k-1} \alpha_{1(k-1)(k-1)}^{+}, \ldots, q_{k-1} \alpha_{r(k-1)(k-1)}^{+}\right)$,

$$
\begin{aligned}
t_{k} & :=q_{k}^{\varepsilon_{k}} \theta_{k} \in q_{k} R_{k-1}, \\
x_{i k} & :=x_{i}+a_{i 1} q_{1}^{\varepsilon_{1}}+\cdots+a_{i k} q_{k}^{\varepsilon_{k}} \in R_{k-1}\left[a_{i k}\right], \\
\eta_{i k} & :=y_{i}+b_{i 1} \theta_{1}^{\varepsilon_{1}}+\cdots+b_{i k} \theta_{k}^{\varepsilon_{k}} \in R_{k-1}\left[q_{k-1} \alpha_{(k-1)(k-1)}^{+}\right]\left[b_{i k}\right], \\
w_{j k} & :=w_{j}+c_{j 1} t_{1}^{\varepsilon_{1}}+\cdots+c_{j k} t_{k}^{\varepsilon_{k}} \in R_{k-1}\left[c_{j k}\right] .
\end{aligned}
$$

\subsection{Numbering, II}

With the notation above, by Taylor's expansion, taking the sums over all $D:=$ $\left(d_{1}, \ldots, d_{n}\right) \in \mathbf{N}_{0}^{n} \backslash\{(0, \ldots, 0)\}$, we get

$$
\begin{aligned}
\phi_{j(k+1) k}^{+} & =\phi_{j k k}^{+}+\sum \frac{\partial^{D} F_{j}}{(\partial Z)^{D}}\left(\underline{\zeta_{k k}}\right)\left(a_{k+1} q_{k+1}^{\varepsilon_{k+1}}\right)^{D}, \\
\phi_{j(k+1) k}^{-} & =\phi_{j k k}^{-}, \\
\phi_{j(k+1)(k+1)}^{+} & =\phi_{j(k+1) k}^{+},
\end{aligned}
$$




$$
\phi_{j(k+1)(k+1)}^{-}=\phi_{j(k+1) k}^{-}+\sum \frac{\partial^{D} F_{j}}{(\partial Z)^{D}} \underline{\left(\zeta_{(k+1) k}\right)}\left(b_{k+1} \theta_{k+1}^{\varepsilon_{k+1}}\right)^{D} .
$$

Hence,

$$
\begin{aligned}
\alpha_{j(k+1) k}^{+}= & \frac{1}{q_{k+1}^{\nu_{k+1}}}\left(\phi_{j(k+1) k}^{+}+w_{j(k+1)}\right) \\
= & \frac{1}{q_{k+1}^{\nu_{k+1}}}\left\{\phi_{j k k}^{+}+\sum \frac{\partial^{D} F_{j}}{(\partial Z)^{D}}\left(\underline{\zeta_{k k}}\right)\left(a_{k+1} q_{k+1}^{\varepsilon_{k+1}}\right)^{D}\right. \\
& \left.+\left(w_{j k}+c_{j(k+1)} t_{k+1}^{\varepsilon_{k+1}}\right)\right\}, \\
\alpha_{j(k+1) k}^{-}= & \frac{1}{\theta_{k}^{\nu_{k}}}\left(\phi_{j k k}^{-}-w_{j k}\right), \quad \\
\alpha_{j(k+1)(k+1)}^{+}= & \frac{1}{q_{k+1}^{\nu_{k+1}}}\left(\phi_{j(k+1) k}^{+}+w_{j(k+1)}\right), \\
\alpha_{j(k+1)(k+1)}^{-}= & \frac{1}{\theta_{k+1}^{\nu_{k+1}}}\left\{\phi_{j(k+1) k}^{-}+\sum \frac{\partial^{D} F_{j}}{(\partial Z)^{D}} \underline{\left(\zeta_{(k+1) k}\right)}\left(b_{k+1} \theta_{k+1}^{\varepsilon_{k+1}}\right)^{D}\right. \\
& \left.-\left(w_{j k}+c_{j(k+1)} t_{k+1}^{\varepsilon_{k+1}}\right)\right\} .
\end{aligned}
$$

Thus,

$$
\begin{aligned}
\alpha_{j k k}^{+}= & \frac{q_{k+1}^{\nu_{k+1}}}{q_{k}^{\nu_{k}}} \alpha_{j(k+1) k}^{+}-c_{j(k+1)} \frac{t_{k+1}^{\varepsilon_{k+1}}}{q_{k}^{\nu_{k}}} \\
& -\frac{1}{q_{k}^{\nu_{k}}} \sum \frac{\partial^{D} F_{j}}{(\partial Z)^{D}}\left(\underline{\zeta_{k k}}\right)\left(a_{k+1} q_{k+1}^{\varepsilon_{k+1}}\right)^{D} \\
= & \frac{q_{k+1}^{\nu_{k+1}}}{q_{k}^{\nu_{k}}} \alpha_{j(k+1) k}^{+}+\frac{q_{k+1}^{\varepsilon_{k+1}}}{q_{k}^{\nu_{k}}} \kappa_{j k k}^{+}, \\
\alpha_{j(k+1) k}^{-}= & \alpha_{j(k+1) k}^{-}, \quad-\frac{1}{\theta_{k}^{\nu_{k}}} \sum \frac{\partial^{D} F_{j}}{(\partial Z)^{D}}\left(\underline{\zeta_{(k+1) k}}\right)\left(b_{k+1} \theta_{k+1}^{\varepsilon_{k+1}}\right)^{D} \\
\alpha_{j(k+1) k}^{-}= & \frac{\theta_{k+1}^{\nu_{k+1}}}{\theta_{k}^{\nu_{k}}} \alpha_{j(k+1)(k+1)}^{-}+c_{j(k+1)} \frac{t_{k+1}^{\varepsilon_{k+1}}}{\theta_{k}^{\nu_{k}}} \\
= & \frac{\theta_{k+1}^{\nu_{k+1}}}{\theta_{k}^{\nu_{k}}} \alpha_{j(k+1)(k+1)}^{-}+\frac{\theta_{k+1}^{\varepsilon_{k+1}}}{\theta_{k}^{\nu_{k}}} \kappa_{j(k+1) k}^{-},
\end{aligned}
$$

where

$$
\begin{aligned}
\kappa_{j k k}^{+} & \in R_{0}\left[\underline{a_{k+1}}, \underline{c_{k+1}}, q_{k+1}^{\varepsilon_{k+1}}, q_{k+1}^{\varepsilon_{k+1}-1} \theta_{k+1}, \underline{\zeta_{k k}}\right] \\
\kappa_{j(k+1) k}^{-} & \in R_{0}\left[\underline{b_{k+1}}, \underline{c_{k+1}}, q_{k+1}^{\varepsilon_{k+1}}, \theta_{k+1}^{\varepsilon_{k+1}}, \underline{\zeta_{(k+1) k}}\right] .
\end{aligned}
$$


Therefore,

$$
\begin{aligned}
& \alpha_{j k k}^{+}=\frac{q_{k+1}^{\nu_{k+1}}}{q_{k}^{\nu_{k}}} \alpha_{j(k+1)(k+1)}^{+}+\frac{q_{k+1}^{\varepsilon_{k+1}}}{q_{k}^{\nu_{k}}} \kappa_{j k k}^{+}, \\
& \alpha_{j k k}^{-}=\frac{\theta_{k+1}^{\nu_{k+1}}}{\theta_{k}^{\nu_{k}}} \alpha_{j(k+1)(k+1)}^{-}+\frac{\theta_{k+1}^{\varepsilon_{k+1}}}{\theta_{k}^{\nu_{k}}} \kappa_{j(k+1) k}^{-},
\end{aligned}
$$

where $\kappa_{j k k}^{+} \in R_{k}\left[\underline{a_{k+1}}, \underline{c_{k+1}}, q_{k-1} \underline{\alpha_{(k-1)(k-1)}^{+}}\right]$and $\kappa_{j(k+1) k}^{-} \in R_{k}\left[\underline{a_{k+1}}, \underline{b_{k+1}}, \underline{c_{k+1}}\right.$, $\left.q_{k} \alpha_{k k}^{+}\right]$.

\subsection{Numbering, III}

Consequently, $\pi_{2}, \pi_{3}, \ldots, \pi_{h}, \ldots$ are written as

$$
\begin{aligned}
\pi_{h} & =r_{h}+\sum r_{h H}\left(q_{h-1} \alpha_{(h-1)(h-1)}^{+}\right)^{H} \\
& =r_{h}+\sum r_{h H}\left(q_{h-1}\left(\frac{q_{h}^{\nu_{h}}}{q_{h-1}^{\nu_{h-1}}} \alpha_{h h}^{+}+\frac{q_{h}^{\varepsilon_{h}}}{q_{h-1}^{\nu_{h-1}}} \kappa_{(h-1)(h-1)}^{+}\right)\right)^{H} \\
& =r_{h}+q_{h}^{\varepsilon_{h}^{\prime}} u_{h h}+\sum r_{h H}\left(\frac{q_{h}^{\nu_{h}-1}}{q_{h-1}^{\nu_{h-1}-1}} q_{h} \alpha_{h h}^{+}\right)^{H},
\end{aligned}
$$

where each $r_{h}, r_{h H} \in R_{h-1}$, where if we take $\varepsilon_{h}$ sufficiently large, then $\varepsilon_{h}^{\prime}$ is large enough and $u_{h h} \in R_{h-1}\left[\underline{a_{h}}, \underline{c_{h}}\right]$ (cf. (2.3.12), (2.4.2)), and where the sums are taken over all $H:=\left(h_{1}, \ldots, h_{r}\right) \in \mathbf{N}_{0}^{r} \backslash\{(0, \ldots, 0)\}$. Hence, if $h \leq m$ and $\varepsilon_{h}, \ldots, \varepsilon_{m}$ are sufficiently large, then

$$
\pi_{h}=r_{h}+q_{h}^{\varepsilon_{h}^{\prime}} u_{h m}+\sum r_{h H}\left(\frac{q_{m}^{\nu_{m}-1}}{q_{h-1}^{\nu_{h-1}-1}} q_{m} \alpha_{m m}^{+}\right)^{H}
$$

with $u_{h m} \in R_{m-1}\left[\underline{a_{m}}, \underline{c_{m}}\right]$.

Putting $s_{h m}:=\pi_{1}\left(r_{2}+q_{2}^{\varepsilon_{2}^{\prime}} u_{2 m}\right) \cdots\left(r_{h}+q_{h}^{\varepsilon_{h}^{\prime}} u_{h m}\right) \in R_{m-1}\left[\underline{a_{m}}, \underline{c_{m}}\right]$, we have

$$
\begin{aligned}
& \theta_{h}=s_{h m}+ \sum t_{h m H}\left(\frac{q_{m}^{\nu_{m}-1}}{q_{h-1}^{\nu_{h-1}-1}} q_{m} \alpha_{m m}^{+}\right)^{H} \\
& \text { with } t_{h m H} \in \pi_{1} R_{m-1}\left[\underline{a_{m}}, \underline{c_{m}}\right] .
\end{aligned}
$$

Thus, when $\varepsilon_{1}, \varepsilon_{2}, \ldots, \varepsilon_{m}$ are suitably large, we see that

$$
\begin{aligned}
\eta_{i m}:=y_{i}+b_{i 1} \theta_{1}^{\varepsilon_{1}}+b_{i 2} \theta_{2}^{\varepsilon_{2}} & +\cdots+b_{i m} \theta_{m}^{\varepsilon_{m}} \\
=y_{i}+b_{i 1} \pi_{1}^{\varepsilon_{1}}+\left(b_{i 2} s_{2 m}^{\varepsilon_{2}}\right. & \left.+\sum v_{i 2 m H}\left(\frac{q_{m}^{\nu_{m}}}{q_{1}^{\nu_{1}}} \alpha_{m m}^{+}\right)^{H}\right)+\cdots \\
& +\left(b_{i m} s_{m m}^{\varepsilon_{m}}+\sum v_{i m m H}\left(\frac{q_{m}^{\nu_{m}}}{q_{m-1}^{\nu_{m}-1}} \alpha_{m m}^{+}\right)^{H}\right) \\
=y_{i}+b_{i 1} \pi_{1}^{\varepsilon_{1}}+b_{i 2} s_{2 m}^{\varepsilon_{2}} & +\cdots+b_{i m} s_{m m}^{\varepsilon_{m}}+\sum \Upsilon_{i m H}\left(\frac{q_{m}^{\nu_{m}}}{q_{m-1}^{\nu_{m-1}}} \alpha_{m m}^{+}\right)^{H},
\end{aligned}
$$


where

$$
\Upsilon_{i m H}=\sum_{h=2}^{m} v_{i h m H}\left(q_{m-1}^{\nu_{m-1}} / q_{h-1}^{\nu_{h-1}}\right)^{H} \in \pi_{1} R_{m-1}\left[\underline{a_{m}}, \underline{b_{m}}, \underline{c_{m}}\right] .
$$

By letting $z_{i \ell m}:=x_{i \ell}+y_{i m}$ with $y_{i m}:=y_{i}+b_{i 1} \pi_{1}^{\varepsilon_{1}}+b_{i 2} s_{2 m}^{\varepsilon_{2}}+\cdots+b_{i m} s_{m m}^{\varepsilon_{m}}$, we get

$$
\zeta_{i \ell m}=x_{i \ell}+\eta_{i m}=z_{i \ell m}+\sum \Upsilon_{i m H}\left(\frac{q_{m}^{\nu_{m}}}{q_{m-1}^{\nu_{m-1}}} \alpha_{m m}^{+}\right)^{H}
$$

When $m \leq \ell$ and $\varepsilon_{2}, \ldots, \varepsilon_{\ell}$ are sufficiently large, we have

$$
\phi_{j \ell m}^{+}=g_{j \ell m}^{+}+\sum_{\hat{\jmath}=1}^{r} d_{j \ell m \hat{\jmath}}^{+} q_{m}^{\nu_{m}} \alpha_{\hat{\jmath} m m}^{+},
$$

where $g_{j \ell m}^{+}:=F_{j}^{+}\left(\underline{z_{\ell m}}\right) \in R_{\ell-1}\left[\underline{a_{\ell}}, \underline{c_{\ell}}\right]$ with $\underline{z_{\ell m}}:=\left(z_{1 \ell m}, \ldots, z_{n \ell m}\right), \underline{a_{\ell}}:=\left(a_{1 \ell}, \ldots\right.$, $\left.a_{n \ell}\right)$, and $\underline{c_{\ell}}:=\left(c_{1 \ell}, \ldots, c_{r \ell}\right)$, and where $\overline{d_{j \ell m \hat{\jmath}}^{+}} \in \pi_{1} \mathfrak{n}_{\ell-1} R_{\ell-1}\left[\underline{a_{\ell}}, \underline{c_{\ell}}\right]$ (cf. (2.1.1)). Also, we get

$$
\phi_{j \ell m}^{-}=g_{j \ell m}^{-}+\sum \Delta_{j \ell m H}^{-}\left(q_{m} \alpha_{m m}^{+}\right)^{H},
$$

where $g_{j \ell m}^{-}:=F_{j}^{-}\left(\underline{z_{\ell m}}\right) \in R_{\ell-1}\left[\underline{a_{\ell}}, \underline{b_{m}}, \underline{c_{\ell}}\right]$ with $\underline{b_{m}}:=\left(b_{1 m}, \ldots, b_{n m}\right)$ and where $\Delta_{j \ell m H}^{-} \in \pi_{1} \mathfrak{n}_{\ell-1} R_{\ell-1}\left[\underline{a_{\ell}}, \underline{b_{m}}, \underline{c_{\ell}}\right]$.

\subsection{Numbering, IV}

Finally, we remark that

$$
\begin{aligned}
\phi_{j k k}^{+}+w_{j k} & =\left(g_{j k k}^{+}+\sum_{\hat{\jmath}=1}^{r} d_{j k k \hat{\jmath}}^{+} q_{k}^{\nu_{k}} \alpha_{\hat{\jmath} k k}^{+}\right)+w_{j k} \\
& =g_{j k k}^{+}+w_{j k}+\sum_{\hat{\jmath}=1}^{r} d_{j k k \hat{\jmath}}^{+}\left(\phi_{\hat{\jmath} k k}^{+}+w_{\hat{\jmath} k}\right) .
\end{aligned}
$$

Thus,

$$
\phi_{j k k}^{+}+w_{j k}=\sum_{\hat{\jmath}=1}^{r} r_{j \hat{\jmath}}^{(k)}\left(g_{\hat{\jmath} k k}^{+}+w_{\hat{\jmath} k}\right)
$$

with an invertible $r \times r$ matrix $\left(r_{j \hat{\jmath}}^{(k)}\right)$ in $R_{k-1}\left(\underline{a_{k}}, \underline{c_{k}}\right)$ (cf. (2.5.4)).

If we put $\alpha_{j k k}^{\Delta}:=\left(1 / q_{k}^{\nu_{k}}\right)\left(g_{j k k}^{+}+w_{j k}\right)$, then $\alpha_{j k k}^{+}=\sum_{\hat{\jmath}=1}^{r} r_{j \hat{\jmath}}^{(k)} \alpha_{\hat{\jmath} k k}^{\Delta}$ and $R_{k}\left[\underline{\alpha_{k k}^{+}}\right]=R_{k}\left[\underline{\alpha_{k k}^{\Delta}}\right]$ where $\underline{\alpha_{k k}^{+}}:=\left(\alpha_{1 k k}^{+}, \ldots, \alpha_{r k k}^{+}\right)$and $\underline{\alpha_{k k}^{\Delta}}:=\left(\alpha_{1 k k}^{\Delta}, \ldots, \alpha_{r k k}^{\Delta}\right)$. Hence,

$$
\begin{aligned}
\phi_{j k k}^{+} & =g_{j k k}^{+}+\sum_{\hat{\jmath}=1}^{r} d_{j k k \hat{\jmath}}^{+} q_{k}^{\nu_{k}} \alpha_{\hat{\jmath} k k}^{+} \\
& =g_{j k k}^{+}+\sum_{\hat{\jmath}=1}^{r} d_{j k k \hat{\jmath}}^{\Delta} q_{k}^{\nu_{k}} \alpha_{\hat{\jmath} k k}^{\Delta},
\end{aligned}
$$




$$
\begin{aligned}
\phi_{j k k}^{-} & =g_{j k k}^{-}+\sum \Delta_{j k k H}^{-}\left(q_{k} \alpha_{k k}^{+}\right)^{H} \\
& =g_{j k k}^{-}+\sum \Delta_{j k k H}^{\nabla}\left(q_{k} \alpha_{k k}^{\Delta}\right)^{H},
\end{aligned}
$$

where $d_{j k k \hat{\jmath}}^{+}, d_{j k k \hat{\jmath}}^{\Delta} \in \pi_{1} \mathfrak{n}_{k-1} R_{k-1}\left(\underline{a_{k}}, \underline{c_{k}}\right)$ and $\Delta_{j k k H}^{-}, \Delta_{j k k H}^{\nabla} \in \pi_{1} \mathfrak{n}_{k} R_{k}$.

\section{Secondary relations}

\subsection{Taylor's formulas, I}

With notation as in previous sections, let $T:=K_{0}\left[Z_{1}, \ldots, Z_{n}, \Delta\right]$ be a polynomial ring in $n+1$ variables over a field $K_{0}$. Take $r$ polynomials in $n$ variables $\underline{Z}:=$ $\left(Z_{1}, \ldots, Z_{n}\right)$ over $K_{0}$ with zero constant term:

$$
F_{1}(\underline{Z}), \ldots, F_{r}(\underline{Z}) \in T_{0}:=K_{0}\left[Z_{1}, \ldots, Z_{n}\right] \subset T .
$$

Let $\underline{V}:=\left(V_{1}, \ldots, V_{r}\right)$, and let $\phi: T[\underline{V}] \rightarrow T[1 / \Delta]$ be a $T$-homomorphism that maps $V_{j}$ to $F_{j}(\underline{Z}) / \Delta$. Then

(3.1.0) $\operatorname{Ker} \phi=\left(\Delta V_{1}-F_{1}(\underline{Z}), \ldots, \Delta V_{r}-F_{r}(\underline{Z}), G_{1}(\underline{Z}, \underline{V}), \ldots, G_{s}(\underline{Z}, \underline{V})\right)$,

where $G_{h}(\underline{Z}, \underline{V}) \in T_{0}[\underline{V}]$ and homogeneous of degree $g_{h}$ in $\underline{V}$

for $h=1, \ldots, s$ (cf. Lemma 1.7). By definition, we have

$$
G_{h}(\underline{Z}, \underline{F} / \Delta) \equiv 0 \quad \text { in } T[1 / \Delta],
$$

where $\underline{F} / \Delta:=\left(F_{1}(\underline{Z}) / \Delta, \ldots, F_{r}(\underline{Z}) / \Delta\right)$. Now let $\underline{\Lambda}:=\left(\Lambda_{1}, \ldots, \Lambda_{r}\right)$ be new variables over $T$. Recall that, by Taylor's expansion (see (2.2.1)), taking the sums over all $E:=\left(e_{1}, \ldots, e_{r}\right) \in \mathbf{N}_{0}^{r} \backslash\{(0, \ldots, 0)\}$, we have

$$
G_{h}(\underline{Z}, \underline{V}+\underline{\Lambda})=G_{h}(\underline{Z}, \underline{V})+\sum \frac{\partial^{E} G_{h}}{(\partial V)^{E}}(\underline{Z}, \underline{V}) \Lambda^{E},
$$

where $\underline{V}+\underline{\Lambda}:=\left(V_{1}+\Lambda_{1}, \ldots, V_{r}+\Lambda_{r}\right)$,

$$
\frac{\partial^{E} G_{h}}{(\partial V)^{E}}(\underline{Z}, \underline{V})=\frac{1}{e_{1} ! \cdots e_{r} !} \cdot \frac{\partial^{e_{1}+\cdots+e_{r}} G_{h}}{\left(\partial V_{1}\right)^{e_{1} \cdots\left(\partial V_{r}\right)^{e_{r}}}}(\underline{Z}, \underline{V}),
$$

and we abbreviate the monomial $\Lambda_{1}^{e_{1}} \cdots \Lambda_{r}^{e_{r}}$ by $\Lambda^{E}$. In (3.1.1), we replace $Z_{i}$ and $F_{j}(\underline{Z})$, respectively, by

$$
\begin{aligned}
Z_{i \ell m} & :=\left(\Delta_{i 0}+\Delta_{i 1}+\cdots+\Delta_{i \ell}\right)+\left(\nabla_{i 0}+\nabla_{i 1}+\cdots+\nabla_{i m}\right), \\
F_{j}\left(\underline{Z_{\ell m}}\right) & :=\left(F_{j}^{+}\left(\underline{Z_{\ell m}}\right)+W_{j \ell}\right)+\left(F_{j}^{-}\left(\underline{Z_{\ell m}}\right)-W_{j \ell}\right),
\end{aligned}
$$

where $Z_{\ell m}:=\left(Z_{1 \ell m}, \ldots, Z_{n \ell m}\right)$. Further, if we replace $\Delta$ by $\Delta_{\ell}$, then we get

$$
\begin{aligned}
G_{h} & \left(\underline{Z_{\ell m}}, \underline{F}\left(\underline{Z_{\ell m}}\right) / \Delta_{\ell}\right) \\
\quad & G_{h}\left(\underline{Z_{\ell m}}, \frac{\left(\underline{F^{+}}\left(\underline{Z_{\ell m}}\right)+\underline{W_{\ell}}\right)+\left(\underline{F^{-}}\left(\underline{Z_{\ell m}}\right)-\underline{W_{\ell}}\right)}{\Delta_{\ell}}\right) \equiv 0
\end{aligned}
$$


in $T\left[\Delta_{\ell}, \underline{W_{\ell}}, \underline{\Delta_{\ell}}, \underline{\nabla_{m}}, 1 / \Delta_{\ell}\right]$. Here we denote

$$
\begin{aligned}
\underline{F}\left(\underline{Z_{\ell m}}\right) / \Delta_{\ell} & :=\left(F_{1}\left(\underline{Z_{\ell m}}\right) / \Delta_{\ell}, \ldots, F_{r}\left(\underline{Z_{\ell m}}\right) / \Delta_{\ell}\right), \\
\underline{W_{\ell}} & :=\left(W_{1 \ell}, \ldots, W_{r \ell}\right), \\
\underline{F^{ \pm}}\left(\underline{Z_{\ell m}}\right) & :=\left(F_{1}^{ \pm}\left(\underline{Z_{\ell m}}\right), \ldots, F_{r}^{ \pm}\left(\underline{Z_{\ell m}}\right)\right), \\
\underline{\Delta_{\ell}} & :=\left(\Delta_{10}, \Delta_{11}, \ldots, \Delta_{1 \ell}, \Delta_{20}, \ldots, \Delta_{n \ell}\right), \quad \text { and } \\
\underline{\nabla_{m}} & :=\left(\nabla_{10}, \nabla_{11}, \ldots, \nabla_{1 m}, \nabla_{20}, \ldots, \nabla_{n m}\right) .
\end{aligned}
$$

Further, if we replace $\Delta$ by $\nabla_{m}$, then we get

$$
\begin{aligned}
& G_{h}\left(\underline{Z_{\ell m}}, \underline{F}\left(\underline{Z_{\ell m}}\right) / \nabla_{m}\right) \\
& \quad=G_{h}\left(\underline{Z_{\ell m}}, \frac{\left(\underline{F^{-}}\left(\underline{Z_{\ell m}}\right)-\underline{W_{m}}\right)+\left(\underline{F^{+}}\left(\underline{Z_{\ell m}}\right)+\underline{W_{m}}\right)}{\nabla_{m}}\right) \equiv 0
\end{aligned}
$$

in $T\left[\nabla_{m}, \underline{W_{\ell}}, \underline{\Delta_{\ell}}, \underline{\nabla_{m}}, 1 / \nabla_{m}\right]$, where

$$
\begin{aligned}
\underline{F}\left(\underline{Z_{\ell m}}\right) / \nabla_{m} & :=\left(F_{1}\left(\underline{Z_{\ell m}}\right) / \nabla_{m}, \ldots, F_{r}\left(\underline{Z_{\ell m}}\right) / \nabla_{m}\right) \quad \text { and } \\
\underline{W_{m}} & :=\left(W_{1 m}, \ldots, W_{r m}\right) .
\end{aligned}
$$

Hence,

$$
\begin{gathered}
G_{h}\left(\underline{Z_{\ell m}}, \frac{\underline{F^{+}}\left(\underline{Z_{\ell m}}\right)+\underline{W_{\ell}}}{\Delta_{\ell}}\right) \\
=-\sum \frac{\partial^{E} G_{h}}{(\partial T)^{E}}\left(\underline{Z_{\ell m}}, \frac{F^{+}\left(\underline{Z_{\ell m}}\right)+\underline{W_{\ell}}}{\Delta_{\ell}}\right)\left(\frac{F^{-}\left(\underline{Z_{\ell m}}\right)-W_{\ell}}{\Delta_{\ell}}\right)^{E}, \\
G_{h}\left(\underline{Z_{\ell m}}, \frac{\underline{F^{-}}\left(\underline{Z_{\ell m}}\right)-\underline{W_{m}}}{\nabla_{m}}\right) \\
\quad=-\sum \frac{\partial^{E} G_{h}}{(\partial V)^{E}}\left(\underline{Z_{\ell m}}, \frac{\underline{F^{-}}\left(\frac{Z_{\ell m}}{\nabla_{m}}\right)-\underline{W_{m}}}{\nabla_{m}}\right)\left(\frac{F^{+}\left(\frac{Z_{\ell m}}{\nabla_{m}}+W_{m}\right.}{{ }_{m}}\right)^{E} .
\end{gathered}
$$

Thus, if we replace $Z_{i k k}, F_{j}^{+}\left(\underline{Z_{k k}}\right), F_{j}^{-}\left(\underline{Z_{k k}}\right), W_{j k}, \Delta_{k}$, and $\nabla_{k}$ by $\zeta_{i k k}, \phi_{j k k}^{+}$, $\phi_{j k k}^{-}, w_{j k}, q_{k}^{\nu_{k}}$, and $\theta_{k}^{\nu_{k}}$, respectively, then we have

$$
\begin{aligned}
& G_{h}\left(\underline{\zeta_{k k}}, \frac{\phi_{k k}^{+}+\underline{w_{k}}}{q_{k}^{\nu_{k}}}\right)=-\sum \frac{\partial^{E} G_{h}}{(\partial V)^{E}}\left(\underline{\zeta_{k k}}, \frac{\phi_{k k}^{+}+\underline{w_{k}}}{q_{k}^{\nu_{k}}}\right)\left(\frac{\phi_{k k}^{-}-w_{k}}{q_{k}^{\nu_{k}}}\right)^{E}, \\
& G_{h}\left(\underline{\zeta_{k k}}, \frac{\phi_{k k}^{-}-\underline{w_{k}}}{\theta_{k}^{\nu_{k}}}\right)=-\sum \frac{\partial^{E} G_{h}}{(\partial V)^{E}}\left(\underline{\zeta_{k k}}, \frac{\phi_{k k}^{-}-\underline{w_{k}}}{\theta_{k}^{\nu_{k}}}\right)\left(\frac{\phi_{k k}^{+}+w_{k}}{\theta_{k}^{\nu_{k}}}\right)^{E} .
\end{aligned}
$$

That is,

$$
\begin{aligned}
& G_{h}\left(\underline{\zeta_{k k}}, \underline{\alpha_{k k}^{+}}\right)=-\sum \frac{\partial^{E} G_{h}}{(\partial V)^{E}}\left(\underline{\zeta_{k k}}, \underline{\alpha_{k k}^{+}}\right)\left(\frac{\theta_{k}^{\nu_{k}}}{q_{k}^{\nu_{k}}} \alpha_{k k}^{-}\right)^{E}, \\
& G_{h}\left(\underline{\zeta_{k k}}, \underline{\alpha_{k k}^{-}}\right)=-\sum \frac{\partial^{E} G_{h}}{(\partial V)^{E}}\left(\underline{\zeta_{k k}}, \underline{\alpha_{k k}^{-}}\right)\left(\frac{q_{k}^{\nu_{k}}}{\theta_{k}^{\nu_{k}}} \alpha_{k k}^{+}\right)^{E} .
\end{aligned}
$$


In the same manner, we get

$$
\begin{aligned}
& G_{h}\left(\underline{\zeta_{(k+1) k}}, \underline{\alpha_{(k+1) k}^{+}}\right) \\
& =-\sum \frac{\partial^{E} G_{h}}{(\partial V)^{E}}\left(\underline{\zeta_{(k+1) k}}, \underline{\alpha_{(k+1) k}^{+}}\right)\left(\frac{\theta_{k}^{\nu_{k}}}{q_{k+1}^{\nu_{k+1}}} \alpha_{(k+1) k}^{-}-c_{k+1} \frac{t_{k+1}^{\varepsilon_{k+1}}}{q_{k+1}^{\nu_{k+1}}}\right)^{E}, \\
& G_{h}\left(\underline{\zeta_{(k+1) k}}, \underline{\alpha_{(k+1) k}^{-}}\right) \\
& =-\sum \frac{\partial^{E} G_{h}}{(\partial V)^{E}}\left(\underline{\zeta_{(k+1) k}}, \underline{\alpha_{(k+1) k}^{-}}\right)\left(\frac{q_{k+1}^{\nu_{k+1}}}{\theta_{k}^{\nu_{k}}} \alpha_{(k+1) k}^{+}-c_{k+1} \frac{t_{k+1}^{\varepsilon_{k+1}}}{\theta_{k}^{\nu_{k}}}\right)^{E}, \\
& G_{h}\left(\underline{\zeta_{(k+1)(k+1)}}, \underline{\alpha_{(k+1)(k+1)}^{+}}\right) \\
& \left.=-\sum \frac{\partial^{E} G_{h}}{(\partial V)^{E}} \underline{\left(\zeta_{(k+1)(k+1)}\right.}, \underline{\alpha_{(k+1)(k+1)}^{+}}\right)\left(\frac{\theta_{k+1}^{\nu_{k+1}}}{q_{k+1}^{\nu_{k+1}}} \alpha_{(k+1)(k+1)}^{-}\right)^{E}, \\
& G_{h}\left(\underline{\zeta_{(k+1)(k+1)}}, \underline{\alpha_{(k+1)(k+1)}^{-}}\right) \\
& \left.=-\sum \frac{\partial^{E} G_{h}}{(\partial V)^{E}} \underline{\left(\zeta_{(k+1)(k+1)}\right.}, \underline{\alpha_{(k+1)(k+1)}^{-}}\right)\left(\frac{q_{k+1}^{\nu_{k+1}}}{\theta_{k+1}^{\nu_{k+1}}} \alpha_{(k+1)(k+1)}^{+}\right)^{E} .
\end{aligned}
$$

\subsection{Taylor's formulas, II}

With the notation above, for a suitable natural number $\mu_{k}$, let

$$
\begin{aligned}
& \beta_{h k k}^{+}:=\frac{1}{\theta_{k}^{\mu_{k}}} G_{h}\left(\underline{\zeta_{k k}}, \underline{\alpha_{k k}^{+}}\right) \\
& =-\frac{1}{\theta_{k}^{\mu_{k}}} \sum \frac{\partial^{E} G_{h}}{(\partial V)^{E}}\left(\underline{\zeta_{k k}}, \underline{\alpha_{k k}^{+}}\right)\left(\frac{\theta_{k}^{\nu_{k}}}{q_{k}^{\nu_{k}}} \alpha_{k k}^{-}\right)^{E}, \\
& \text { (3.2.2) } \quad \beta_{h k k}^{-}:=\frac{1}{q_{k}^{\mu_{k}}} G_{h}\left(\underline{\zeta_{k k}}, \underline{\alpha_{k k}^{-}}\right) \\
& =-\frac{1}{q_{k}^{\mu_{k}}} \sum \frac{\partial^{E} G_{h}}{(\partial V)^{E}}\left(\underline{\zeta_{k k}}, \underline{\alpha_{k k}^{-}}\right)\left(\frac{q_{k}^{\nu_{k}}}{\theta_{k}^{\nu_{k}}} \alpha_{k k}^{+}\right)^{E}, \\
& \beta_{h(k+1) k}^{+}:=\frac{1}{\theta_{k}^{\mu_{k}}} G_{h}\left(\underline{\zeta_{(k+1) k}}, \underline{\alpha_{(k+1) k}^{+}}\right) \\
& =-\frac{1}{\theta_{k}^{\mu_{k}}} \sum \frac{\partial^{E} G_{h}}{(\partial V)^{E}}\left(\underline{\zeta_{(k+1) k}}, \underline{\alpha_{(k+1) k}^{+}}\right) \\
& \times\left(\frac{\theta_{k}^{\nu_{k}}}{q_{k+1}^{\nu_{k+1}}} \alpha_{(k+1) k}^{-}-c_{k+1} \frac{t_{k+1}^{\varepsilon_{k+1}}}{q_{k+1}^{\nu_{k+1}}}\right)^{E}, \\
& \beta_{h(k+1) k}^{-}:=\frac{1}{q_{k+1}^{\mu_{k+1}}} G_{h}\left(\underline{\zeta_{(k+1) k}}, \underline{\alpha_{(k+1) k}^{-}}\right) \\
& =-\frac{1}{q_{k+1}^{\mu_{k+1}}} \sum \frac{\partial^{E} G_{h}}{(\partial V)^{E}}\left(\underline{\zeta_{(k+1) k}}, \underline{\alpha_{(k+1) k}^{-}}\right)
\end{aligned}
$$




$$
\begin{aligned}
& \times\left(\frac{q_{k+1}^{\nu_{k+1}}}{\theta_{k}^{\nu_{k}}} \alpha_{(k+1) k}^{+}-c_{k+1} \frac{t_{k+1}^{\varepsilon_{k+1}}}{\theta_{k}^{\nu_{k}}}\right)^{E}, \\
& \beta_{h(k+1)(k+1)}^{+}:=\frac{1}{\theta_{k+1}^{\mu_{k+1}}} G_{h}\left(\underline{\zeta_{(k+1)(k+1)}}, \underline{\alpha_{(k+1)(k+1)}^{+}}\right) \\
& \left.=-\frac{1}{\theta_{k+1}^{\mu_{k+1}}} \sum \frac{\partial^{E} G_{h}}{(\partial V)^{E}} \underline{\left(\zeta_{(k+1)(k+1)}\right.}, \underline{\alpha_{(k+1)(k+1)}^{+}}\right) \\
& \times\left(\frac{\theta_{k+1}^{\nu_{k+1}}}{q_{k+1}^{\nu_{k+1}}} \alpha_{(k+1)(k+1)}^{-}\right)^{E}, \\
& \beta_{h(k+1)(k+1)}^{-}:=\frac{1}{q_{k+1}^{\mu_{k+1}}} G_{h}\left(\underline{\zeta_{(k+1)(k+1)}}, \underline{\alpha_{(k+1)(k+1)}^{-}}\right) \\
& =-\frac{1}{q_{k+1}^{\mu_{k+1}}} \sum \frac{\partial^{E} G_{h}}{(\partial V)^{E}}\left(\underline{\zeta_{(k+1)(k+1)}}, \underline{\alpha_{(k+1)(k+1)}^{-}}\right) \\
& \times\left(\frac{q_{k+1}^{\nu_{k+1}}}{\theta_{k+1}^{\nu_{k+1}}} \alpha_{(k+1)(k+1)}^{+}\right)^{E} .
\end{aligned}
$$

Thus,

$$
\begin{aligned}
& \beta_{h k k}^{+}=-\frac{1}{\theta_{k}^{\mu_{k}}} \sum \frac{\partial^{E} G_{h}}{(\partial V)^{E}}\left(\underline{\zeta_{(k+1) k}}-\underline{a_{k+1}} q_{k+1}^{\varepsilon_{k+1}}, \frac{q_{k+1}^{\nu_{k+1}}}{q_{k}^{\nu_{k}}} \underline{\alpha_{(k+1) k}^{+}}+\frac{q_{k+1}^{\varepsilon_{k+1}}}{q_{k}^{\nu_{k}}} \underline{\kappa_{k k}^{+}}\right) \\
& \times\left\{\frac{q_{k+1}^{\nu_{k+1}}}{q_{k}^{\nu_{k}}}\left(\frac{\theta_{k}^{\nu_{k}}}{q_{k+1}^{\nu_{k+1}}} \alpha_{(k+1) k}^{-}-c_{k+1} \frac{t_{k+1}^{\varepsilon_{k+1}}}{q_{k+1}^{\nu_{k+1}}}\right)+\frac{q_{k+1}^{\nu_{k+1}}}{q_{k}^{\nu_{k}}} c_{k+1} \frac{t_{k+1}^{\varepsilon_{k+1}}}{q_{k+1}^{\nu_{k+1}}}\right\}^{E} \\
& =-\left(\frac{q_{k+1}^{\nu_{k+1}}}{q_{k}^{\nu_{k}}}\right)^{g_{h}} \frac{1}{\theta_{k}^{\mu_{k}}} \sum \frac{\partial^{E} G_{h}}{(\partial V)^{E}}\left(\underline{\zeta_{(k+1) k}}, \underline{\alpha_{(k+1) k}^{+}}\right) \\
& \times\left(\frac{\theta_{k}^{\nu_{k}}}{q_{k+1}^{\nu_{k+1}}} \alpha_{(k+1) k}^{-}-c_{k+1} \frac{t_{k+1}^{\varepsilon_{k+1}}}{q_{k+1}^{\nu_{k+1}}}\right)^{E} \\
& +\frac{q_{k+1}^{\varepsilon_{k+1}}}{q_{k}^{\nu_{k} g_{h}}}\left(\frac{\theta_{k+1}^{\varepsilon_{k+1}}}{\theta_{k}^{\mu_{k}}} \delta_{h k k}^{+}+\frac{\theta_{k}^{\nu_{k}}}{\theta_{k}^{\mu_{k}}} \sum_{j=1}^{r} \lambda_{h j k k}^{+} \alpha_{j(k+1) k}^{-}\right) \\
& =-\left(\frac{q_{k+1}^{\nu_{k+1}}}{q_{k}^{\nu_{k}}}\right)^{g_{h}} \beta_{h(k+1) k}^{+}+\frac{q_{k+1}^{\varepsilon_{k+1}}}{q_{k}^{\nu_{k} g_{h}}}\left(\frac{\theta_{k+1}^{\varepsilon_{k+1}}}{\theta_{k}^{\mu_{k}}} \delta_{h k k}^{+}+\frac{\theta_{k}^{\nu_{k}}}{\theta_{k}^{\mu_{k}}} \sum_{j=1}^{r} \lambda_{h j k k}^{+} \alpha_{j(k+1) k}^{-}\right),
\end{aligned}
$$

where each $\delta_{h k k}^{+} \in R_{0}\left[\underline{a_{k+1}}, \underline{c_{k+1}}, p_{k+1}, q_{k}, \theta_{k+1}^{\varepsilon_{k+1}}, \underline{\zeta_{k k}}, q_{k+1}^{\nu_{k+1}} \underline{\alpha_{(k+1)(k+1)}^{+}}, \theta_{k}^{\nu_{k}} \underline{\alpha_{k k}^{-}}\right]$ and $\lambda_{h j k k}^{+} \in R_{0}\left[\underline{a_{k+1}}, p_{k+1}, q_{k}, \underline{\zeta_{k k}}, q_{k+1}^{\nu_{k+1}} \alpha_{(k+1)(k+1)}^{+}, \theta_{k}^{\nu_{k}} \underline{\alpha_{k k}^{-}}\right] \overline{(\mathrm{cf.}(3.1 .0))}$. Then we have

$$
\begin{aligned}
\beta_{h k k}^{-} & =\frac{1}{q_{k}^{\mu_{k}}} G_{h}\left(\underline{\zeta_{(k+1) k}}-\underline{a_{k+1}} q_{k+1}^{\varepsilon_{k+1}}, \underline{\alpha_{(k+1) k}^{-}}\right) \\
& =\frac{q_{k+1}^{\mu_{k+1}}}{q_{k}^{\mu_{k}}} \frac{1}{q_{k+1}^{\mu_{k+1}}} G_{h}\left(\underline{\zeta_{(k+1) k}}, \underline{\alpha_{(k+1) k}^{-}}\right)
\end{aligned}
$$




$$
\begin{aligned}
& +\frac{1}{q_{k}^{\mu_{k}}} \sum \frac{\partial^{D} G_{h}}{(\partial Z)^{D}}\left(\underline{\zeta_{(k+1) k}}, \underline{\alpha_{(k+1) k}^{-}}\right)\left(-a_{k+1} q_{k+1}^{\varepsilon_{k+1}}\right)^{D} \\
= & \frac{q_{k+1}^{\mu_{k+1}}}{q_{k}^{\mu_{k}}} \beta_{h(k+1) k}^{-}+\frac{q_{k+1}^{\varepsilon_{k+1}}}{q_{k}^{\mu_{k}}} \delta_{h k k}^{-},
\end{aligned}
$$

where $\delta_{h k k}^{-} \in R_{0}\left[\underline{a_{k+1}}, q_{k+1}^{\varepsilon_{k+1}}, \underline{\zeta_{(k+1) k}}\right]\left[\underline{\alpha_{k k}^{-}}\right]$and homogeneous in $\underline{\alpha_{k k}^{-}}$. We have

$$
\begin{aligned}
& \beta_{h(k+1) k}^{+}= \frac{1}{\theta_{k}^{\mu_{k}}} G_{h}\left(\underline{\zeta_{(k+1)(k+1)}}-\underline{b_{k+1}} \theta_{k+1}^{\varepsilon_{k+1}}, \underline{\alpha_{(k+1)(k+1)}^{+}}\right) \\
&=\left.\frac{\theta_{k+1}^{\mu_{k+1}}}{\theta_{k}^{\mu_{k}}} \frac{1}{\theta_{k+1}^{\mu_{k+1}}} G_{h} \underline{\left(\underline{\zeta_{(k+1)(k+1)}}\right.}, \underline{\alpha_{(k+1)(k+1)}^{+}}\right) \\
&\left.\quad+\frac{1}{\theta_{k}^{\mu_{k}}} \sum \frac{\partial^{D} G_{h}}{(\partial Z)^{D}} \underline{\left(\zeta_{(k+1)(k+1)}\right.}, \underline{\alpha_{(k+1)(k+1)}^{+}}\right)\left(-b_{k+1} \theta_{k+1}^{\left.\varepsilon_{k+1}\right)^{D}}\right. \\
&=\frac{\theta_{k+1}^{\mu_{k+1}}}{\theta_{k}^{\mu_{k}}} \beta_{h(k+1)(k+1)}^{+}+\frac{\theta_{k+1}^{\varepsilon_{k+1}}}{\theta_{k}^{\mu_{k}}} \delta_{h(k+1) k}^{+},
\end{aligned}
$$

where $\delta_{h(k+1) k}^{+} \in R_{0}\left[\underline{b_{k+1}}, \theta_{k+1}^{\varepsilon_{k+1}}, \underline{\zeta_{(k+1)(k+1)}}\right]\left[\underline{\alpha_{(k+1)(k+1)}^{+}}\right]$and homogeneous in $\alpha_{(k+1)(k+1)}^{+}$. We have

$$
\begin{aligned}
& \beta_{h(k+1) k}^{-}=-\frac{1}{q_{k+1}^{\mu_{k+1}}} \sum \frac{\partial^{E} G_{h}}{(\partial V)^{E}}\left(\underline{\zeta_{(k+1)(k+1)}}-\underline{b_{k+1}} \theta_{k+1}^{\varepsilon_{k+1}},\right. \\
& \left.\frac{\theta_{k+1}^{\nu_{k+1}}}{\theta_{k}^{\nu_{k}}} \underline{\alpha_{(k+1)(k+1)}^{-}}+\frac{\theta_{k+1}^{\varepsilon_{k+1}}}{\theta_{k}^{\nu_{k}}} \underline{\kappa_{(k+1) k}^{-}}\right) \\
& \times\left(\frac{\theta_{k+1}^{\nu_{k+1}}}{\theta_{k}^{\nu_{k}}} \frac{q_{k+1}^{\nu_{k+1}}}{\theta_{k+1}^{\nu_{k+1}}} \alpha_{(k+1)(k+1)}^{+}-c_{k+1} \frac{t_{k+1}^{\varepsilon_{k+1}}}{\theta_{k}^{\nu_{k}}}\right)^{E} \\
& =-\left(\frac{\theta_{k+1}^{\nu_{k+1}}}{\theta_{k}^{\nu_{k}}}\right)^{g_{h}} \frac{1}{q_{k+1}^{\mu_{k+1}}} \sum \frac{\partial^{E} G_{h}}{(\partial V)^{E}}\left(\underline{\zeta_{(k+1)(k+1)}},\right. \\
& \left.\underline{\alpha_{(k+1)(k+1)}^{-}}\right)\left(\frac{q_{k+1}^{\nu_{k+1}}}{\theta_{k+1}^{\nu_{k+1}}} \alpha_{(k+1)(k+1)}^{+}\right)^{E} \\
& +\frac{\theta_{k+1}^{\varepsilon_{k+1}}}{\theta_{k}^{\nu_{k} g_{h}}}\left(\frac{q_{k+1}^{\varepsilon_{k+1}}}{q_{k+1}^{\mu_{k+1}}} \delta_{h(k+1) k}^{-}+\frac{q_{k+1}^{\nu_{k+1}}}{q_{k+1}^{\mu_{k+1}}} \sum_{j=1}^{r} \lambda_{h j(k+1) k}^{-} \alpha_{j(k+1)(k+1)}^{+}\right) \\
& =-\left(\frac{\theta_{k+1}^{\nu_{k+1}}}{\theta_{k}^{\nu_{k}}}\right)^{g_{h}} \beta_{h(k+1)(k+1)}^{-} \\
& +\frac{\theta_{k+1}^{\varepsilon_{k+1}}}{\theta_{k}^{\nu_{k} g_{h}}}\left(\frac{q_{k+1}^{\varepsilon_{k+1}}}{q_{k+1}^{\mu_{k+1}}} \delta_{h(k+1) k}^{-}+\frac{q_{k+1}^{\nu_{k+1}}}{q_{k+1}^{\mu_{k+1}}} \sum_{j=1}^{r} \lambda_{h j(k+1) k}^{-} \alpha_{j(k+1)(k+1)}^{+}\right),
\end{aligned}
$$

where 


$$
\begin{aligned}
& \delta_{h(k+1) k}^{-} \in R_{0}\left[\underline{b_{k+1}}, \underline{c_{k+1}}, q_{k+1}^{\varepsilon_{k+1}}, \pi_{k+1}, \theta_{k+1}, \underline{\zeta_{(k+1) k}},\right. \\
& \left.q_{k+1}^{\nu_{k+1}} \underline{\alpha_{(k+1)(k+1)}^{+}}, \theta_{k+1}^{\nu_{k+1}} \underline{\alpha_{(k+1)(k+1)}^{-}}\right], \\
& \lambda_{h j(k+1) k}^{-} \in R_{0}\left[\underline{b_{k+1}}, \pi_{k+1}, \theta_{k}, \underline{\zeta_{(k+1) k}}, q_{k+1}^{\nu_{k+1}} \underline{\alpha_{(k+1)(k+1)}^{+}}, \theta_{k+1}^{\nu_{k+1}} \underline{\alpha_{(k+1)(k+1)}^{-}}\right] .
\end{aligned}
$$

\subsection{Taylor's formulas, III}

Summarizing the above, we have

$$
\begin{aligned}
\beta_{h k k}^{+}=-\left(\frac{q_{k+1}^{\nu_{k+1}}}{q_{k}^{\nu_{k}}}\right)^{g_{h}}\left(\frac{\theta_{k+1}^{\mu_{k+1}}}{\theta_{k}^{\mu_{k}}} \beta_{h(k+1)(k+1)}^{+}+\frac{\theta_{k+1}^{\varepsilon_{k+1}}}{\theta_{k}^{\mu_{k}}} \delta_{h(k+1) k}^{+}\right) \\
+\frac{q_{k+1}^{\varepsilon_{k+1}}}{q_{k}^{\nu_{k} g_{h}}}\left(\frac{\theta_{k+1}^{\varepsilon_{k+1}}}{\theta_{k}^{\mu_{k}}} \delta_{h k k}^{+}+\frac{\theta_{k}^{\nu_{k}}}{\theta_{k}^{\mu_{k}}} \sum \lambda_{h j k k}^{+} \alpha_{j(k+1) k}^{-}\right), \\
\beta_{h k k}^{-}=-\frac{q_{k+1}^{\mu_{k+1}}}{q_{k}^{\mu_{k}}}\left(\frac{\theta_{k+1}^{\nu_{k+1}}}{\theta_{k}^{\nu_{k}}}\right)^{g_{h}} \beta_{h(k+1)(k+1)}^{-} \\
+\frac{\theta_{k+1}^{\varepsilon_{k+1}}}{\theta_{k}^{\nu_{k} g_{h}}}\left(\frac{q_{k+1}^{\varepsilon_{k+1}}}{q_{k}^{\mu_{k}}} \delta_{h(k+1) k}^{-}+\frac{q_{k+1}^{\nu_{k+1}}}{q_{k}^{\mu_{k}}} \sum \lambda_{h j(k+1) k}^{-} \alpha_{j(k+1)(k+1)}^{+}\right) \\
+\frac{q_{k+1}^{\varepsilon_{k+1}}}{q_{k}^{\mu_{k}}} \delta_{h k k}^{-},
\end{aligned}
$$

where

$$
\begin{gathered}
\delta_{h k k}^{+}, \lambda_{h j k k}^{+} \in R_{k}\left[\underline{\left.\alpha_{k k}^{+}\right]}\left[\underline{a_{k+1}}, \underline{c_{k+1}}\right],\right. \\
\delta_{h k k}^{-} \in R_{k}\left[\underline{\left.\alpha_{k k}^{+}\right]}\left[\underline{\underline{a_{k+1}}}\right]\left[\underline{\alpha_{k k}^{-}}\right] \text {and homogeneous in } \underline{\alpha_{k k}^{-}},\right. \\
\delta_{h(k+1) k}^{+} \in R_{k}\left[\underline{\alpha_{k k}^{+}}\right]\left[\underline{a_{k+1}}, \underline{b_{k+1}}\right]\left[\underline{\alpha_{(k+1)(k+1)}^{+}}\right] \text {and } \\
\text { homogeneous in } \underline{\underline{\alpha_{(k+1)(k+1)}^{+}}}, \\
\delta_{h(k+1) k}^{-}, \lambda_{h j(k+1) k}^{-} \in R_{k+1}\left[\underline{\alpha_{k k}^{+}}\right] .
\end{gathered}
$$

\section{Prime elements}

As mentioned in the Introduction, we consider polynomials in $n$ variables over a countable field $K_{0}$ without constant term: $F_{1}(\underline{Z}), \ldots, F_{r}(\underline{Z}) \in K_{0}\left[Z_{1}, \ldots, Z_{n}\right]=$ : $T_{0}$, and we assume the following.

\section{ASSUMPTION 4.1}

The $F_{j}$ 's satisfy the following:

$$
\bigcup_{\nu \geq 1} \operatorname{Ass}\left(T_{0} /\left(F_{1}, \ldots, F_{r}\right)^{\nu}\right)=\operatorname{Ass}\left(T_{0} /\left(F_{1}, \ldots, F_{r}\right)\right) .
$$

For each $\mathfrak{P} \in \operatorname{Ass}\left(T_{0} /\left(F_{1}, \ldots, F_{r}\right)\right)$, there exists a proper subset

$$
\left\{i_{1}, \ldots, i_{t}\right\} \text { of }\{1,2, \ldots, n\} \text { such that } \mathfrak{P} \subset \mathfrak{Q}:=\left(Z_{i_{1}}, \ldots, Z_{i_{t}}\right) T_{0} .
$$


From now on, we fully use the notation in Sections 2 and 3.

\section{DEFINITION 4.2}

Let $\mathfrak{q}_{k}^{+}, \mathfrak{q}_{k}^{-}$and $\mathfrak{q}_{k}:=\mathfrak{q}_{k}^{+}+\mathfrak{q}_{k}^{-}$be ideals of $R_{k}\left[\alpha_{k k}^{+}\right]$as follows:

$$
\begin{aligned}
\mathfrak{q}_{k}^{+} & :=\left(\phi_{1 k k}^{+}+w_{1 k}, \ldots, \phi_{r k k}^{+}+w_{r k}\right), \\
\mathfrak{q}_{k}^{-} & :=\left(\phi_{1 k k}^{-}-w_{1 k}, \ldots, \phi_{r k k}^{-}-w_{r k}\right) .
\end{aligned}
$$

We use $\phi_{j k k}^{+}$from (2.3.7), $\phi_{j k k}^{-}$from (2.3.8), and $w_{j k}$ from (2.3.5).

\section{DEFINITION 4.3}

Let $\mathfrak{q}_{k}^{\Delta}, \mathfrak{q}_{k}^{\nabla}$, and $\mathfrak{q}_{k}^{\diamond}$ be ideals of $R_{k}$ as follows:

$$
\begin{aligned}
\mathfrak{q}_{k}^{\Delta} & :=\left(g_{1 k k}^{+}+w_{1 k}, \ldots, g_{r k k}^{+}+w_{r k}\right), \\
\mathfrak{q}_{k}^{\nabla} & :=\left(g_{1 k k}^{-}-w_{1 k}, \ldots, g_{r k k}^{-}-w_{r k}\right), \\
\mathfrak{q}_{k}^{\diamond} & :=\mathfrak{q}_{k}^{\Delta}+\mathfrak{q}_{k}^{\nabla} .
\end{aligned}
$$

We remark that $\mathfrak{q}_{k}^{\Delta} R_{k}\left[\alpha_{k k}^{+}\right]=\mathfrak{q}_{k}^{+}$by (2.6.2) (cf. (2.6.4)).

Next let $\tilde{R}_{k}:=R_{k}\left[\alpha_{k k}^{+}\right]_{\mathfrak{N}_{k}}$ be a local ring with maximal ideal $\tilde{\mathfrak{n}}_{k}:=\mathfrak{N}_{k} \tilde{R}_{k}$, where $\mathfrak{N}_{k}:=\left(z_{0}, \underline{z}, \underline{w}, \underline{\alpha_{k k}^{+}}\right)$is a maximal ideal of $R_{k}\left[\underline{\alpha_{k k}^{+}}\right]$(cf. Lemma 6.2).

\section{DEFINITION 4.4}

Let $\tilde{\mathfrak{q}}_{k}^{+}=\tilde{\mathfrak{q}}_{k}^{\Delta}, \tilde{\mathfrak{q}}_{k}^{-}$, and $\tilde{\mathfrak{q}}_{k}^{\nabla}$ be ideals of $\tilde{R}_{k}$ as follows:

$$
\begin{aligned}
\tilde{\mathfrak{q}}_{k}^{+} & :=\mathfrak{q}_{k}^{+} \tilde{R}_{k}\left[1 / q_{k}\right] \cap \tilde{R}_{k}=\mathfrak{q}_{k}^{\Delta} \tilde{R}_{k}\left[1 / q_{k}\right] \cap \tilde{R}_{k}=: \tilde{\mathfrak{q}}_{k}^{\Delta}, \\
\tilde{\mathfrak{q}}_{k}^{-} & :=\mathfrak{q}_{k}^{-} \tilde{R}_{k}\left[1 / q_{k}\right] \cap \tilde{R}_{k}, \\
\tilde{\mathfrak{q}}_{k}^{\nabla} & :=\mathfrak{q}_{k}^{\nabla} \tilde{R}_{k}\left[1 / q_{k}\right] \cap \tilde{R}_{k} .
\end{aligned}
$$

Further, let

$$
\begin{aligned}
\tilde{\mathfrak{q}}_{k} & :=\tilde{\mathfrak{q}}_{k}^{+}+\tilde{\mathfrak{q}}_{k}^{-}, \\
\tilde{\mathfrak{q}}_{k}^{\diamond} & :=\tilde{\mathfrak{q}}_{k}^{\Delta}+\tilde{\mathfrak{q}}_{k}^{\nabla} .
\end{aligned}
$$

\section{LEMMA 4.5}

Assume that $p_{i} \notin \mathfrak{q}_{k-1}^{\Delta}$ for each $i \leq k$. Then

$$
R_{k} / \mathfrak{q}_{k}^{\Delta} \stackrel{\cong}{\longrightarrow} \tilde{R}_{k} / \tilde{\mathfrak{q}}_{k}^{+} \quad \text { and } \quad \tilde{\mathfrak{q}}_{k}^{+}=\left(\alpha_{1 k k}^{+}, \ldots, \alpha_{r k k}^{+}\right) .
$$

Here $\tilde{\mathfrak{q}}_{k}^{+}$is a prime ideal generated by an $\tilde{R}_{k}$-sequence $\alpha_{1 k k}^{+}, \ldots, \alpha_{r k k}^{+}$, and $p_{i} \notin \tilde{\mathfrak{q}}_{k}^{+}$ for each $i \leq k$. Suppose, in addition, that $p_{1} \tilde{R}_{k-1}+\tilde{\mathfrak{q}}_{k-1}^{+}, \ldots, p_{k} \tilde{R}_{k-1}+\tilde{\mathfrak{q}}_{k-1}^{+}$are distinct prime ideals of $\tilde{R}_{k-1}$. Then $p_{1} \tilde{R}_{k}+\tilde{\mathfrak{q}}_{k}^{+}, \ldots, p_{k} \tilde{R}_{k}+\tilde{\mathfrak{q}}_{k}^{+}$are distinct prime ideals of $\tilde{R}_{k}$ and, whenever $\varepsilon_{k} \geq e_{i}$,

$$
\left(p_{i}^{e_{i}} \tilde{R}_{k}+\tilde{\mathfrak{q}}_{k}^{+}\right) \cap \tilde{R}_{k-1}=p_{i}^{e_{i}} \tilde{R}_{k-1}+\tilde{\mathfrak{q}}_{k-1}^{+} .
$$


Proof

We first remark that $\mathfrak{q}_{k}^{\Delta}$ is a prime ideal generated by the elements $g_{1 k k}^{+}+$ $w_{1 k}, \ldots, g_{r k k}^{+}+w_{r k}$ that form a part of a regular system of parameters of the regular local ring $R_{k}$ (cf. (2.1.1)), and by assumption, it is clear that $p_{i} \notin \mathfrak{q}_{k}^{\Delta}$ for each $i \leq k$. By definition, we have the following commutative diagram with canonical surjective morphisms:

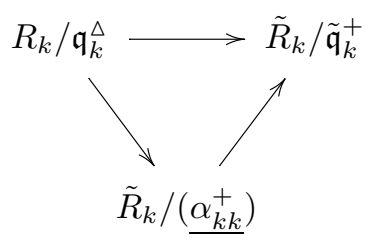

Indeed, the three surjective morphisms above are isomorphisms, because we have the following canonical inclusions:

$$
R_{k} / \mathfrak{q}_{k}^{\Delta} \subset \tilde{R}_{k} / \tilde{\mathfrak{q}}_{k}^{+} \subset\left(\tilde{R}_{k} / \mathfrak{q}_{k}^{\Delta} \tilde{R}_{k}\right)\left[1 / q_{k}\right]=\left(\left(R_{k} / \mathfrak{q}_{k}^{\Delta}\right)\left[1 / q_{k}\right]\right)_{\mathfrak{N}_{k}} .
$$

In particular, $\tilde{\mathfrak{q}}_{k}^{+}=\left(\underline{\alpha_{k k}^{+}}\right) \tilde{R}_{k}$. This implies that $\tilde{\mathfrak{q}}_{k}^{+}$is a prime ideal generated by an $\tilde{R}_{k}$-sequence $\alpha_{1 k k}^{+}, \ldots, \alpha_{r k k}^{+}$and that $p_{i} \notin \tilde{\mathfrak{q}}_{k}^{+}$. Consequently, if $\varepsilon_{k} \geq e_{i}$, then we have

$$
\begin{aligned}
\tilde{R}_{k} /\left(p_{i}^{e_{i}}, \tilde{\mathfrak{q}}_{k}^{+}\right) & \cong R_{k} /\left(p_{i}^{e_{i}}, \mathfrak{q}_{k}^{\Delta}\right) \\
& =R_{k} /\left(p_{i}^{e_{i}}, \mathfrak{q}_{k-1}^{\Delta}\right) \\
& \cong\left(\tilde{R}_{k-1} /\left(p_{i}^{e_{i}}, \tilde{\mathfrak{q}}_{k-1}^{+}\right)\right)\left(\underline{a_{k}}, \underline{b_{k}}, \underline{c_{k}}\right) .
\end{aligned}
$$

\section{LEMMA 4.6}

With notation and the assumptions as in Definitions 4.3 and 4.4 and Lemma 4.5, assume further that $\pi_{1}, q_{k}$ form an $\left(R_{k} / \mathfrak{q}_{k}^{\diamond}\right)$-sequence. Then

$$
\pi_{1} \notin \bigcup \operatorname{Ass}\left(\tilde{R}_{k} / \tilde{\mathfrak{q}}_{k}\right)=\bigcup \operatorname{Ass}\left(\tilde{R}_{k} / \tilde{\mathfrak{q}}_{k}^{\diamond}\right),
$$

where $\bigcup \operatorname{Ass}\left(\tilde{R}_{k} / \tilde{\mathfrak{q}}_{k}\right)$ denotes the union of the prime ideals in $\operatorname{Ass}\left(\tilde{R}_{k} / \tilde{\mathfrak{q}}_{k}\right)$ for a fixed $k \in \mathbf{N}$. Hence, (4.6.1) implies that $\pi_{1}$ is a non-zero divisor for $\tilde{R}_{k} / \tilde{\mathfrak{q}}_{k}$.

Proof

Because $R_{k} / \mathfrak{q}_{k}^{\Delta} \cong \tilde{R}_{k} / \tilde{\mathfrak{q}}_{k}^{+}$by (4.5.1), we have (cf. (2.3.8))

$$
R_{k} / \mathfrak{q}_{k}^{\diamond} \stackrel{\cong}{\longrightarrow} \tilde{R}_{k} /\left(\tilde{\mathfrak{q}}_{k}^{+}+\mathfrak{q}_{k}^{\nabla} \tilde{R}_{k}\right)=\tilde{R}_{k} /\left(\tilde{\mathfrak{q}}_{k}^{\Delta}+\mathfrak{q}_{k}^{-} \tilde{R}_{k}\right) .
$$

Thus, we get canonical surjections

$$
\tilde{R}_{k} /\left(\tilde{\mathfrak{q}}_{k}^{+}+\mathfrak{q}_{k}^{-} \tilde{R}_{k}\right) \longrightarrow \tilde{R}_{k} / \tilde{\mathfrak{q}}_{k} \quad \text { and } \quad \tilde{R}_{k} /\left(\tilde{\mathfrak{q}}_{k}^{\Delta}+\mathfrak{q}_{k}^{\nabla} \tilde{R}_{k}\right) \longrightarrow \tilde{R}_{k} / \tilde{\mathfrak{q}}_{k}^{\diamond}
$$

However, the canonical inclusion $R_{k} / \mathfrak{q}_{k}^{\diamond} \subset\left(R_{k} / \mathfrak{q}_{k}^{\diamond}\right)\left[1 / q_{k}\right]$ and the isomorphism above show that these surjective morphisms are isomorphisms. Namely,

$$
R_{k} / \mathfrak{q}_{k}^{\diamond} \stackrel{\cong}{\longrightarrow} \tilde{R}_{k} / \tilde{\mathfrak{q}}_{k}=\tilde{R}_{k} / \tilde{\mathfrak{q}}_{k}^{\diamond}
$$

This gives the assertion. 


\section{LEMMA 4.7}

With assumptions as in Lemma 4.5, assume further that $\pi_{j} \notin \tilde{\mathfrak{q}}_{k-1}^{-}$for each $j \leq k$. Then $\tilde{\mathfrak{q}}_{k}^{-}$is a prime ideal and $\pi_{j} \notin \tilde{\mathfrak{q}}_{k}^{-}$for each $j \leq k$.

Proof

By assumption, first we note that $\pi_{j} \notin \mathfrak{q}_{\ell-1}^{-} R_{\ell-1}\left[1 / q_{\ell-1}\right]$ for $j \leq \ell \leq k$ (cf. (2.3.13), (2.4.1)). Next we show by induction that

$$
\left(\phi_{1 \ell \ell}^{-}-w_{1 \ell}, \ldots, \phi_{h \ell \ell}^{-}-w_{h \ell}\right) R_{\ell}\left[1 / q_{\ell}\right] \text { is a prime ideal generated }
$$

by an $R_{\ell}\left[1 / q_{\ell}\right]$-sequence for each $h \leq r$ and $0 \leq \ell \leq k$.

Indeed, the case $\ell=0$ is true, because $F_{1}(\underline{z})-w_{1}, \ldots, F_{h}(\underline{z})-w_{h}$ form a subregular system of parameters of $R_{0}$ (cf. (2.1.0), (2.3.1), (2.3.2)). Hence, we only show the claim for the case $\ell=k$, assuming that

$$
\left(\phi_{1(k-1)(k-1)}^{-}-w_{1(k-1)}, \ldots, \phi_{h(k-1)(k-1)}^{-}-w_{h(k-1)}\right) R_{k-1}\left[1 / q_{k-1}\right] \text { is a prime }
$$

ideal generated by an $R_{k-1}\left[1 / q_{k-1}\right]$-sequence for each $h \leq r$.

But, the assumption above implies that $\theta_{k}, \phi_{1(k-1)(k-1)}^{-}-w_{1(k-1)}, \ldots$, $\phi_{r(k-1)(k-1)}^{-}-w_{r(k-1)}$ form an $R_{k-1}\left[1 / q_{k-1}\right]$-sequence (cf. [8, Lemma 1.1]). Thus, $\mathfrak{q}_{k}^{-} R_{k}\left[1 / q_{k}\right]$ is a prime ideal and $\pi_{j} \notin \mathfrak{q}_{k}^{-} R_{k}\left[1 / q_{k}\right]$. In fact, this results from

$$
\begin{aligned}
\phi_{j k k}^{-}-w_{j k} & =\left(\phi_{j(k-1)(k-1)}^{-}-w_{j(k-1)}\right)+\left\{H_{j}\left(\underline{\zeta_{k(k-1)}}, \underline{b_{k}}, \theta_{k}\right) \theta_{k}^{\varepsilon_{k}}-c_{j k}\left(q_{k}^{\varepsilon_{k}} \theta_{k}\right)^{\varepsilon_{k}}\right\} \\
& =\left(\phi_{j(k-1)(k-1)}^{-}-w_{j(k-1)}\right)+\left(H_{j}-c_{j k} q_{k}^{\varepsilon_{k}^{2}}\right) \theta_{k}^{\varepsilon_{k}},
\end{aligned}
$$

where (cf. (2.4.1), Lemma 1.2)

$$
\begin{aligned}
H_{j}\left(\underline{\zeta_{k(k-1)}}, \underline{b_{k}}, \theta_{k}\right) & =\frac{1}{\theta_{k}^{\varepsilon_{k}}} \sum \frac{\partial^{D} F_{j}}{(\partial Z)^{D}}\left(\underline{\zeta_{k(k-1)}}\right)\left(b_{k} \theta_{k}^{\varepsilon_{k}}\right)^{D} \\
& \in R_{k-1}\left[\underline{\alpha_{(k-1)(k-1)}^{+}}\right]\left[\underline{a_{k}}, \underline{b_{k}}\right] \subset R_{k-1}\left[\underline{a_{k}}, \underline{b_{k}}\right]\left[1 / q_{k}\right] .
\end{aligned}
$$

Therefore, $\tilde{\mathfrak{q}}_{k}^{-}$is a prime ideal and $\pi_{j} \notin \tilde{\mathfrak{q}}_{k}^{-}$for each $j \leq k$.

\section{LEMMA 4.8}

With assumptions as in Lemmas 4.6 and 4.7, assume further that

$$
\begin{aligned}
& q_{k}, z_{1 k k}, \ldots, z_{n k k} \text { form an }\left(R_{k} / \mathfrak{q}_{k}^{\Delta}\right) \text {-sequence, } \\
& q_{k}, z_{1 k k}, \ldots, z_{n k k} \text { form an }\left(R_{k} / \mathfrak{q}_{k}^{\nabla}\right) \text {-sequence. }
\end{aligned}
$$

Then

$$
\tilde{\mathfrak{q}}_{k}^{-}=\left(\mathfrak{q}_{k}^{-}, G_{1}\left(\underline{\zeta_{k k}}, \underline{\alpha_{k k}^{+}}\right), \ldots, G_{s}\left(\underline{\zeta_{k k}}, \underline{\alpha_{k k}^{+}}\right)\right) .
$$

Proof

First we show that $\tilde{\mathfrak{q}}_{k}^{-} \supset\left(\mathfrak{q}_{k}^{-}, G_{1}\left(\underline{\zeta_{k k}}, \alpha_{k k}^{+}\right), \ldots, G_{s}\left(\underline{\zeta_{k k}}, \alpha_{k k}^{+}\right)\right)$. Indeed, denoting by $T:=K_{0}\left[Z_{1}, \ldots, Z_{n}, \Delta\right]$ a polynomial ring in $n+1$ variables over a field $K_{0}$, we 
consider a $K_{0}$-algebra homomorphism

$\varphi_{0}: T \rightarrow R_{k}\left[\underline{\alpha_{k k}^{+}}\right]$that maps $Z_{i}$ to $\zeta_{i k k}$ for $i=1, \ldots, n$ and $\Delta$ to $q_{k}^{\nu_{k}}$.

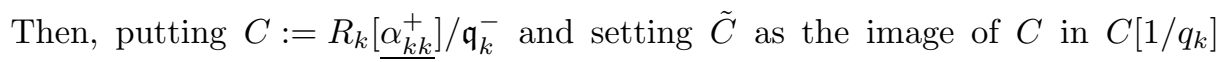
with canonical projections $\pi: R_{k}\left[\alpha_{k k}^{+}\right] \rightarrow C$ and $\tilde{\pi}: R_{k}\left[\alpha_{k k}^{+}\right] \rightarrow \tilde{C}$, we have the following commutative diagram, because $\left.\left(\phi_{j k k}^{+}+w_{j k}\right)+\overline{\left(\phi_{j k k}^{-}\right.}-w_{j k}\right)=F_{j}\left(\underline{\zeta_{k k}}\right)$ and because the image of $\alpha_{j k k}^{+}$in $\tilde{C}$ can be identified with that of $F_{j}\left(\underline{\zeta_{k k}}\right) / \underline{q_{k}^{\nu_{k}}}$ :

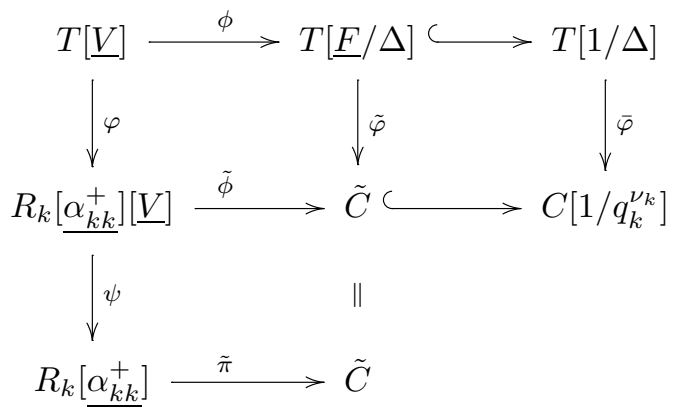

where $\varphi:=\varphi_{0} \otimes K_{0}[\underline{V}], \bar{\varphi}:=\left(\pi \circ \varphi_{0}\right) \otimes_{K_{0}[\Delta]} K_{0}[\Delta, 1 / \Delta]$, and $\psi$ is an $R_{k}\left[\underline{\alpha_{k k}^{+}}\right]$algebra homomorphism with $V_{j} \mapsto \alpha_{j k k}^{+}$. Further, $\phi$ is a $T$-homomorphism with $V_{j} \mapsto F_{j}(\underline{Z}) / \Delta, \tilde{\phi}$ is an $R_{k}\left[\underline{\alpha_{k k}^{+}}\right]$-algebra homomorphism that maps $V_{j}$ to the image of $\alpha_{j k k}^{+}$in $\tilde{C}$, and $\tilde{\varphi}$ is the $K_{0}$-algebra homomorphism induced by $\varphi_{0}$ that maps $F_{j}(\underline{Z}) / \Delta$ to the image of $\alpha_{j k k}^{+}$in $\tilde{C}$. Then, by Lemma 1.7,

$\operatorname{Ker} \phi=\left(\Delta V_{1}-F_{1}(\underline{Z}), \ldots, \Delta V_{r}-F_{r}(\underline{Z}), G_{1}(\underline{Z}, \underline{V}), \ldots, G_{s}(\underline{Z}, \underline{V})\right)$.

Hence, by definition, $\tilde{\mathfrak{q}}_{k}^{-} \supset \operatorname{Ker} \tilde{\pi} \supset\left(\mathfrak{q}_{k}^{-}, G_{1}\left(\underline{\zeta_{k k}}, \alpha_{k k}^{+}\right), \ldots, G_{s}\left(\underline{\zeta_{k k}}, \alpha_{k k}^{+}\right)\right)$.

Now we prove (4.8.1). Note that $\mathfrak{q}_{k}^{\Delta}$ is generated by an $\left.\overline{\left(R_{k}\right.} / \overline{q_{k} R_{k}}\right)$-sequence (cf. (4.3.1)). Further, we have (cf. (2.3.8), (4.3.2))

$$
\begin{aligned}
\mathfrak{q}_{k}^{\diamond} & :=\mathfrak{q}_{k}^{\Delta}+\mathfrak{q}_{k}^{\nabla} \\
& =\mathfrak{q}_{k}^{\Delta}+\left(F_{1}\left(\underline{z_{k k}}\right), \ldots, F_{r}\left(\underline{z_{k k}}\right)\right) \\
& =\left(F_{1}\left(\underline{z_{k k}}\right), \ldots, F_{r}\left(\underline{z_{k k}}\right)\right)+\mathfrak{q}_{k}^{\nabla}
\end{aligned}
$$

and $z_{1 k k}, \ldots, z_{n k k}$ form an $\left(R_{k} /\left(q_{k}, \mathfrak{q}_{k}^{\Delta}\right)\right)$-sequence. Thus, by Lemma 1.11, we see that

$$
\bigcup \bigcup_{\nu \geq 1} \operatorname{Ass}\left(R_{k} /\left(q_{k},\left(\mathfrak{q}_{k}^{\Delta}\right)^{\nu}\right)\right)=\bigcup \operatorname{Ass}\left(R_{k} /\left(q_{k}, \mathfrak{q}_{k}^{\Delta}\right)\right) \subset \bigcup \operatorname{Ass}\left(R_{k} /\left(q_{k}, \mathfrak{q}_{k}^{\diamond}\right)\right),
$$

where the first union is the union of the prime ideals in $\operatorname{Ass}\left(R_{k} /\left(q_{k},\left(\mathfrak{q}_{k}^{\Delta}\right)^{\nu}\right)\right)$ of all the $\nu$ for a fixed $k \in \mathbf{N}$ (cf. (0.1.1)), and $\bigcup \operatorname{Ass}\left(R_{k} /\left(q_{k}, \mathfrak{q}_{k}^{\diamond}\right)\right)$ denotes the union of the prime ideals in $\operatorname{Ass}\left(R_{k} /\left(q_{k}, \mathfrak{q}_{k}^{\diamond}\right)\right)$ for a fixed $k \in \mathbf{N}$. Hence, by Lemma 4.6

$$
\begin{aligned}
\pi_{1} & \notin \bigcup \bigcup_{\nu \geq 1} \operatorname{Ass}\left(R_{k} /\left(q_{k},\left(\mathfrak{q}_{k}^{\Delta}\right)^{\nu}\right)\right) \\
& =\bigcup \operatorname{Ass}\left(R_{k}\left[\underline{\alpha_{k k}^{\Delta}}\right] / q_{k} R_{k}\left[\underline{\alpha_{k k}^{\Delta}}\right]\right) \cap R_{k}
\end{aligned}
$$




$$
=\bigcup \operatorname{Ass}\left(R_{k}\left[\underline{\alpha_{k k}^{+}}\right] / q_{k} R_{k}\left[\underline{\alpha_{k k}^{+}}\right]\right) \cap R_{k} .
$$

Here $\bigcup \operatorname{Ass}\left(R_{k}\left[\underline{\alpha_{k k}^{\Delta}}\right] / q_{k} R_{k}\left[\underline{\alpha_{k k}^{\Delta}}\right]\right) \cap R_{k}$ means the union of the prime ideals in the set

$$
\left\{\mathfrak{p}=\mathfrak{P} \cap R_{k} \mid \mathfrak{P} \in \operatorname{Ass}\left(R_{k}\left[\underline{\alpha_{k k}^{\Delta}}\right] / q_{k} R_{k}\left[\underline{\alpha_{k k}^{\Delta}}\right]\right)\right\} .
$$

Then, letting $\bar{\alpha}_{j k k}^{+}, \bar{\alpha}_{j k k}^{\Delta}$ be the images of $\alpha_{j k k}^{+}, \alpha_{j k k}^{\Delta}$ in the field of quotients $Q\left(\bar{R}_{k}\right)$ of $\bar{R}_{k}=R_{k} / \pi_{1} R_{k}$, we get the following isomorphisms (cf. (1.9.1)):

$$
\begin{aligned}
& \bar{R}_{k}\left[\underline{\left.\bar{\alpha}_{k k}^{+}\right]} \cong\left(R_{k} / \pi_{1} R_{k}\right) \otimes R_{k}\left[\underline{\alpha_{k k}^{+}}\right]\right. \\
&=\left(R_{k} / \pi_{1} R_{k}\right) \otimes R_{k}\left[\underline{\alpha_{k k}^{\Delta}}\right] \cong \bar{R}_{k}\left[\underline{\bar{\alpha}_{k k}^{\Delta}}\right] .
\end{aligned}
$$

On the other hand, by assumption (cf. (4.8.2))

$$
\bigcup_{\nu \geq 1} \operatorname{Ass}\left(R_{k} /\left(q_{k}, \mathfrak{q}_{k}^{\nabla},\left(\mathfrak{q}_{k}^{\Delta}\right)^{\nu}\right)\right)=\operatorname{Ass}\left(R_{k} /\left(q_{k}, \mathfrak{q}_{k}^{\diamond}\right)\right) .
$$

Thus, denoting by $\tilde{\alpha}_{j k k}^{\Delta}$ the image of $\alpha_{j k k}^{\Delta}$ in $Q\left(R_{k} / \mathfrak{q}_{k}^{\nabla}\right)$, we have (cf. Lemma 1.8)

$$
\begin{aligned}
\pi_{1} & \notin \bigcup \operatorname{Ass}\left(\left(R_{k} / \mathfrak{q}_{k}^{\nabla}\right)\left[\underline{\tilde{\alpha}_{k k}^{\Delta}}\right] / q_{k}\left(R_{k} / \mathfrak{q}_{k}^{\nabla}\right)\left[\underline{\tilde{\alpha}_{k k}^{\Delta}}\right]\right) \cap R_{k} \\
& =\bigcup \bigcup_{\nu \geq 1} \operatorname{Ass}\left(R_{k} /\left(q_{k}, \mathfrak{q}_{k}^{\nabla},\left(\mathfrak{q}_{k}^{\Delta}\right)^{\nu}\right)\right) .
\end{aligned}
$$

By mapping $V_{j}$ to $\tilde{\alpha}_{j k k}^{\Delta}$, consider an $R_{k}$-algebra isomorphism

$$
R_{k}\left[V_{1}, \ldots, V_{r}\right] / J \stackrel{\cong}{\rightarrow}\left(R_{k} / \mathfrak{q}_{k}^{\nabla}\right)\left[\tilde{\alpha}_{1 k k}^{\Delta}, \ldots, \tilde{\alpha}_{r k k}^{\Delta}\right] \subset \tilde{R}_{k} / \tilde{\mathfrak{q}}_{k}^{\nabla} .
$$

Then we have (cf. (4.8.2), Lemma 1.7)

$$
\begin{aligned}
J=\left(\mathfrak{q}_{k}^{\nabla}, q_{k}^{\nu_{k}} V_{1}-\left(g_{1 k k}^{+}+w_{1 k}\right), \ldots\right. \\
\\
\left.\quad \ldots, q_{k}^{\nu_{k}} V_{r}-\left(g_{r k k}^{+}+w_{r k}\right), G_{1}\left(\underline{z_{k k}}, \underline{V}\right), \ldots, G_{s}\left(\underline{z_{k k}}, \underline{V}\right)\right) .
\end{aligned}
$$

Hence,

$$
\begin{aligned}
& \tilde{R}_{k} /\left(\pi_{1}, \mathfrak{q}_{k}^{-}, \underline{G}\left(\underline{\zeta_{k k}}, \underline{\alpha_{k k}^{+}}\right)\right) \cong\left(\bar{R}_{k}\left[\underline{\left.\bar{\alpha}_{k k}^{+}\right]}\right)_{\overline{\mathfrak{N}}_{k}} /\left(\overline{\mathfrak{q}}_{k}^{-}, \underline{\bar{G}}\left(\underline{\bar{\zeta}_{k k}}, \underline{\bar{\alpha}_{k k}^{+}}\right)\right)\right. \\
& \cong\left(\bar{R}_{k}\left[\underline{\bar{\alpha}_{k k}^{\Delta}}\right]\right)_{\overline{\mathfrak{N}}_{k}} /\left(\overline{\mathfrak{q}}_{k}^{\nabla}, \underline{\bar{G}}\left(\underline{\bar{z}_{k k}}, \underline{\bar{\alpha}_{k k}^{\Delta}}\right)\right) \\
& \cong \tilde{R}_{k} /\left(\pi_{1}, \tilde{\mathfrak{q}}_{k}^{\nabla}\right) \text {, }
\end{aligned}
$$

where $\overline{\mathfrak{q}}_{k}^{-}, \bar{G}_{h}\left(\underline{\bar{\zeta}_{k k}}, \bar{\alpha}_{k k}^{+}\right), \overline{\mathfrak{q}}_{k}^{\nabla}$, and $\bar{G}_{h}\left(\underline{\bar{z}_{k k}}, \bar{\alpha}_{k k}^{\Delta}\right)$ are the images of $\mathfrak{q}_{k}^{-}, G_{h}\left(\underline{\zeta_{k k}}, \underline{\alpha_{k k}^{+}}\right)$, $\mathfrak{q}_{k}^{\nabla}$, and $G_{h}\left(\underline{z_{k k}}, \overline{\alpha_{k k}^{\Delta}}\right)$ in $Q\left(\tilde{R}_{k} / \pi_{1} \tilde{R}_{k}\right)=Q\left(\left(\bar{R}_{k}\left[\bar{\alpha}_{k k}^{+}\right]\right)_{\overline{\mathfrak{N}}_{k}}\right)$ (cf. (4.8.4), (2.6.3), (2.6.1)). Thus, $\left(\pi_{1}, \tilde{\mathfrak{q}}_{k}^{-}\right)=\left(\pi_{1}, \mathfrak{q}_{k}^{-}, \underline{G}\left(\underline{\zeta_{k k}}, \underline{\alpha_{k k}^{+}}\right)\right)$, because (cf. (4.8.3), (4.4.1))

$$
\begin{aligned}
q_{k} & \notin \bigcup \operatorname{Ass}\left(R_{k}\left[\alpha_{k k}^{\Delta}\right] /\left(\pi_{1}, \mathfrak{q}_{k}^{\nabla}, \underline{G}\left(\underline{z_{k k}}, \underline{\alpha_{k k}^{\Delta}}\right)\right)\right) \\
& =\bigcup \operatorname{Ass}\left(R_{k}\left[\underline{\alpha_{k k}^{+}}\right] /\left(\pi_{1}, \mathfrak{q}_{k}^{-}, \underline{G}\left(\underline{z_{k k}}, \underline{\alpha_{k k}^{+}}\right)\right)\right) .
\end{aligned}
$$

Therefore, $\tilde{\mathfrak{q}}_{k}^{-}=\left(\mathfrak{q}_{k}^{-}, \underline{G}\left(\underline{\zeta_{k k}}, \underline{\alpha_{k k}^{+}}\right)\right)$, because $\tilde{\mathfrak{q}}_{k}^{-}: \pi_{1}=\tilde{\mathfrak{q}}_{k}^{-}(\mathrm{cf} .(4.7 .1))$. 
Finally, note that in the proof above we have also proved

$$
\begin{aligned}
\left(\pi_{1}, \tilde{\mathfrak{q}}_{k}^{-}\right) & =\left(\pi_{1}, \tilde{\mathfrak{q}}_{k}^{\nabla}\right), \text { and } \\
\tilde{\mathfrak{q}}_{k}^{\nabla} & =\left(\mathfrak{q}_{k}^{\nabla}, G_{1}\left(\underline{z_{k k}}, \underline{\alpha_{k k}^{\Delta}}\right), \ldots, G_{s}\left(\underline{z_{k k}}, \underline{\alpha_{k k}^{\Delta}}\right)\right) \text { is a prime ideal. }
\end{aligned}
$$

\section{LEMMA 4.9}

With the notation and assumptions from Lemma 4.8, we have

$$
\tilde{\mathfrak{q}}_{k}^{+} \cap \tilde{\mathfrak{q}}_{k}^{-}=\left(\alpha_{i k k}^{+}\left(\phi_{j k k}^{-}-w_{j k}\right), G_{h}\left(\underline{\zeta_{k k}}, \underline{\alpha_{k k}^{+}}\right)\right)
$$

for $i=1, \ldots, n, j=1, \ldots, r$, and $h=1, \ldots, s$, where $\underline{\zeta_{k k}}:=\left(\zeta_{1 k k}, \ldots, \zeta_{n k k}\right)$ and $\underline{\alpha_{k k}^{+}}:=\left(\alpha_{1 k k}^{+}, \ldots, \alpha_{r k k}^{+}\right)$.

\section{Proof}

Let $I:=\tilde{\mathfrak{q}}_{k}^{+} \cap \tilde{\mathfrak{q}}_{k}^{-}$, and let $J:=\tilde{\mathfrak{q}}_{k}^{\Delta} \cap \tilde{\mathfrak{q}}_{k}^{\nabla}$. Then by (4.6.1), (4.7.1), and (4.8.4), we get the following commutative diagrams whose rows are exact:

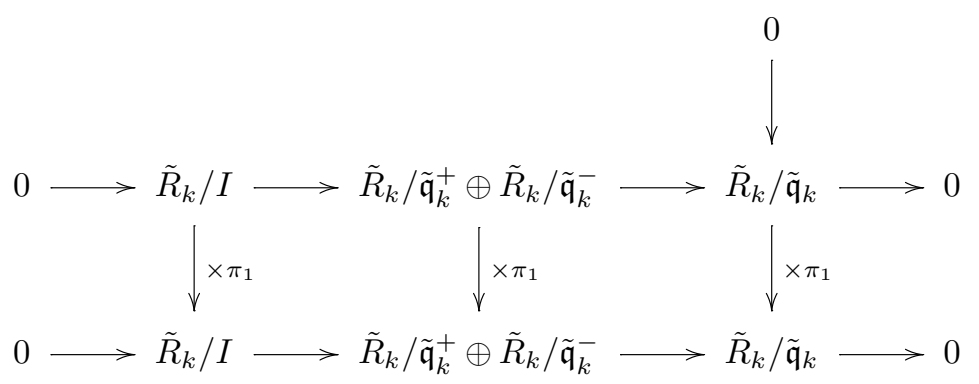

and

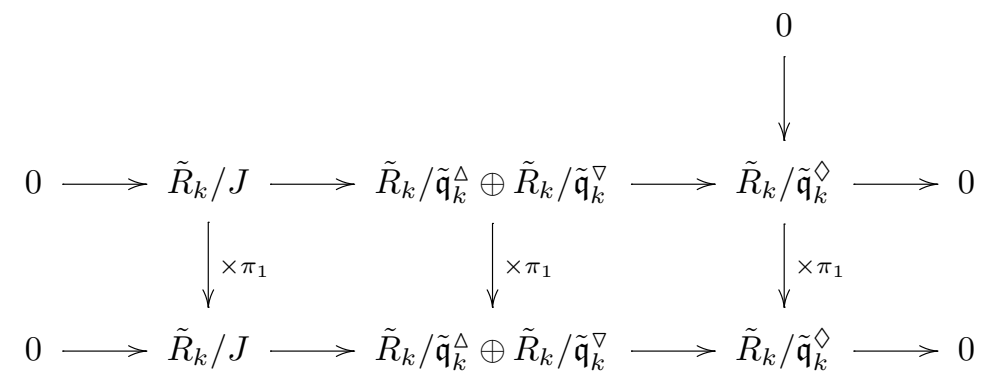

Hence, we also get the following commutative diagram whose rows are exact (cf. $(4.8 .5))$ :

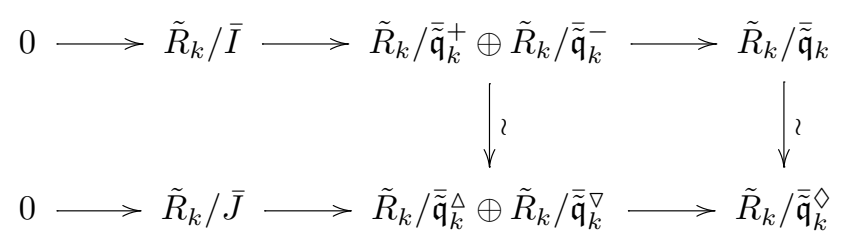

where 
$\bar{I}:=\left(\pi_{1}, I\right), \quad \overline{\tilde{\mathfrak{q}}}_{k}^{+}:=\left(\pi_{1}, \tilde{\mathfrak{q}}_{k}^{+}\right), \quad \overline{\tilde{\mathfrak{q}}}_{k}^{-}:=\left(\pi_{1}, \tilde{\mathfrak{q}}_{k}^{-}\right), \quad \overline{\tilde{\mathfrak{q}}}_{k}:=\overline{\tilde{\mathfrak{q}}}_{k}^{+}+\overline{\tilde{\mathfrak{q}}}_{k}^{-}=\left(\pi_{1}, \tilde{\mathfrak{q}}_{k}\right)$,

$\bar{J}:=\left(\pi_{1}, J\right), \quad \overline{\tilde{\mathfrak{q}}}_{k}^{\Delta}:=\left(\pi_{1}, \tilde{\mathfrak{q}}_{k}^{\Delta}\right), \quad \overline{\tilde{\mathfrak{q}}}_{k}^{\nabla}:=\left(\pi_{1}, \tilde{\mathfrak{q}}_{k}^{\nabla}\right), \quad \overline{\tilde{\mathfrak{q}}}_{k}^{\diamond}:=\overline{\tilde{\mathfrak{q}}}_{k}^{\Delta}+\overline{\tilde{\mathfrak{q}}}_{k}^{\nabla}=\left(\pi_{1}, \tilde{\mathfrak{q}}_{k}^{\diamond}\right)$.

Thus, it suffices to show that $J=\left(\alpha_{i k k}^{\Delta}\left(g_{j k k}^{-}-w_{j k}\right), G_{h}\left(\underline{z_{k k}}, \underline{\alpha_{k k}^{\Delta}}\right)\right)$ for $i=1, \ldots, n$, $j=1, \ldots, r$, and $h=1, \ldots, s$. This follows from Lemma 1.10.

\section{LEMMA 4.10}

With assumptions as in Lemma 4.8, we have

$$
\begin{aligned}
& \operatorname{Ass}\left(\left(\tilde{R}_{k} / \tilde{\mathfrak{q}}_{k}^{-}\right) / q_{k}\left(\tilde{R}_{k} / \tilde{\mathfrak{q}}_{k}^{-}\right)\right) \cap R_{k} \\
& \quad=\operatorname{Ass}\left(\tilde{R}_{k} /\left(q_{k}, \tilde{\mathfrak{q}}_{k}\right)\right) \cap R_{k}, \\
& \operatorname{Ass}\left(\left(\tilde{R}_{k} / \tilde{\mathfrak{q}}_{k}^{-}\right) / q_{k}\left(\tilde{R}_{k} / \tilde{\mathfrak{q}}_{k}^{-}\right)\right) \cap R_{k}\left[q_{k} \underline{\alpha_{k k}^{+}}\right] \\
& \quad=\operatorname{Ass}\left(\tilde{R}_{k} /\left(q_{k}, \tilde{\mathfrak{q}}_{k}\right)\right) \cap R_{k}\left[q_{k} \underline{\alpha_{k k}^{+}}\right] .
\end{aligned}
$$

Here $\operatorname{Ass}\left(\tilde{R}_{k} /\left(q_{k}, \tilde{\mathfrak{q}}_{k}\right)\right) \cap R_{k}:=\left\{\mathfrak{p}=\mathfrak{P} \cap R_{k} \mid \mathfrak{P} \in \operatorname{Ass}\left(\tilde{R}_{k} /\left(q_{k}, \tilde{\mathfrak{q}}_{k}\right)\right)\right\}$.

Proof

First we show that there exists an $R_{k}$-algebra isomorphism

$$
\tilde{R}_{k} /\left(q_{k}, \tilde{\mathfrak{q}}_{k}^{-}\right) \stackrel{\cong}{\longrightarrow} \tilde{R}_{k} /\left(q_{k}, \tilde{\mathfrak{q}}_{k}^{\nabla}\right) .
$$

Indeed, we have an $R_{k}$-algebra isomorphism $R_{k}\left[\underline{V^{+}}\right] / J^{+} \cong R_{k}\left[\alpha_{k k}^{+}\right]$, mapping $V_{j}^{+}$to $\alpha_{j k k}^{+}$as in (4.8.4), and $J^{+}=\left(q_{k}^{\nu_{k}} V_{j}^{+}-\left(\phi_{j k k}^{+}+w_{j k}\right)\right)$. Further, $\tilde{\mathfrak{q}}_{k}^{-}=$ $\left(\mathfrak{q}_{k}^{-}, G_{h}\left(\underline{\zeta_{k k}}, \underline{\alpha_{k k}^{+}}\right)\right)$, where (cf. (4.8.1), (2.6.4), (2.5.3))

$$
\begin{aligned}
\mathfrak{q}_{k}^{-} & =\left(\phi_{j k k}^{-}-w_{j k}\right)=\left(g_{j k k}^{-}+\sum \underline{\Delta_{j k k H}^{-}}\left(q_{k} \alpha_{k k}^{+}\right)^{H}-w_{j k}\right), \\
G_{h}\left(\underline{\zeta_{k k}}, \underline{\alpha_{k k}^{+}}\right) & =G_{h}\left(\underline{z_{k k}}+\sum \underline{\Upsilon_{k H}}\left(\left(q_{k}^{\nu_{k}} / q_{k-1}^{\nu_{k-1}}\right) \alpha_{k k}^{+}\right)^{H}, \underline{\alpha_{k k}^{+}}\right) .
\end{aligned}
$$

Moreover, we have an $R_{k}$-algebra isomorphism $R_{k}\left[\underline{V^{\Delta}}\right] / J^{\Delta} \cong R_{k}\left[\underline{\alpha_{k k}^{\Delta}}\right]$ with $V_{j}^{\Delta} \mapsto$ $\alpha_{j k k}^{\Delta}$ and $J^{\Delta}=\left(q_{k}^{\nu_{k}} V_{j}^{\Delta}-\left(g_{j k k}^{+}+w_{j k}\right)\right)$ for $j=1, \ldots, r$. Also, we have $\tilde{\mathfrak{q}}_{k}^{\nabla}=$ $\left(\mathfrak{q}_{k}^{\nabla}, G_{h}\left(\underline{z_{k k}} \underline{\alpha_{k k}^{\Delta}}\right)\right)$, where $\mathfrak{q}_{k}^{\nabla}:=\left(g_{j k k}^{-}-w_{j k}\right)$ (cf. (4.8.5)). Thus, we have $R_{k}$-algebra isomorphisms

$$
\begin{gathered}
\left(R_{k}\left[\underline{V^{+}}\right] /\left(q_{k}, \phi_{j k k}^{+}+w_{j k}, g_{j k k}^{-}-w_{j k}, G_{h}\left(\underline{z_{k k}}, \underline{V^{+}}\right)\right)\right)_{\mathfrak{N}_{V+}} \stackrel{\cong}{\longrightarrow} \tilde{R}_{k} /\left(q_{k}, \tilde{\mathfrak{q}}_{k}^{-}\right), \\
\left(R_{k}\left[\underline{V^{\Delta}}\right] /\left(q_{k}, g_{j k k}^{+}+w_{j k}, g_{j k k}^{-}-w_{j k}, G_{h}\left(\underline{z_{k k}}, \underline{V^{\Delta}}\right)\right)\right)_{\mathfrak{N}_{V^{\Delta}}} \stackrel{\cong}{\longrightarrow} \tilde{R}_{k} /\left(q_{k}, \tilde{\mathfrak{q}}_{k}^{\nabla}\right),
\end{gathered}
$$

where $\mathfrak{N}_{V^{+}}:=\left(z_{0}, \underline{z}, \underline{w}, \underline{V^{+}}\right)$is a maximal ideal of $R_{k}\left[\underline{V^{+}}\right]$and $\mathfrak{N}_{V^{\Delta}}:=\left(z_{0}, \underline{z}, \underline{w}\right.$, $\underline{V^{\Delta}}$ ) is a maximal ideal of $R_{k}\left[\underline{V}^{\Delta}\right]$. Therefore, we get the assertion (4.10.3) (cf. $(2.6 .2))$. Now (4.10.1) results from

$$
\begin{gathered}
\operatorname{Ass}\left(\left(\tilde{R}_{k} / \tilde{\mathfrak{q}}_{k}^{-}\right) / q_{k}\left(\tilde{R}_{k} / \tilde{\mathfrak{q}}_{k}^{-}\right)\right) \cap R_{k} \\
=\operatorname{Ass}\left(\tilde{R}_{k} /\left(q_{k}, \tilde{\mathfrak{q}}_{k}^{-}\right)\right) \cap R_{k} \\
=\operatorname{Ass}\left(\tilde{R}_{k} /\left(q_{k}, \tilde{\mathfrak{q}}_{k}^{\nabla}\right)\right) \cap R_{k}
\end{gathered}
$$




$$
\begin{aligned}
& =\operatorname{Ass}\left(\left(\left(R_{k} / \mathfrak{q}_{k}^{\nabla}\right)\left[\underline{\tilde{\alpha}_{k k}^{\Delta}}\right] / q_{k}\left(R_{k} / \mathfrak{q}_{k}^{\nabla}\right)\left[\underline{\tilde{\alpha}_{k k}^{\Delta}}\right]\right)_{\mathfrak{N}_{k}}\right) \cap R_{k} \\
& =\bigcup_{\nu \geq 1} \operatorname{Ass}\left(R_{k} /\left(q_{k}, \mathfrak{q}_{k}^{\nabla},\left(\mathfrak{q}_{k}^{\Delta}\right)^{\nu}\right)\right) \\
& =\operatorname{Ass}\left(R_{k} /\left(q_{k}, \mathfrak{q}_{k}^{\nabla}, \mathfrak{q}_{k}^{\Delta}\right)\right) \\
& =\operatorname{Ass}\left(R_{k} /\left(q_{k}, \mathfrak{q}_{k}^{\diamond}\right)\right) \\
& =\operatorname{Ass}\left(\tilde{R}_{k} /\left(q_{k}, \tilde{\mathfrak{q}}_{k}\right)\right) \cap R_{k},
\end{aligned}
$$

where $\tilde{\alpha}_{j k k}^{\Delta}$ is the image of $\alpha_{j k k}^{\Delta}$ in $Q\left(R_{k} / \mathfrak{q}_{k}^{\nabla}\right)$ (cf. (4.8.4), (4.1.1), (4.6.2)). Finally, (4.10.2) follows from (4.10.1), because $q_{k} \tilde{R}_{k} \cap R_{k}\left[q_{k} \alpha_{k k}^{+}\right]$contains all the $q_{k} \alpha_{j k k}^{+}$'s and because $\left.R_{k}\left[q_{k} \underline{\alpha_{k k}^{+}}\right] /\left(q_{k} \underline{\alpha_{k k}^{+}}\right) R_{k}\left[q_{k} \underline{\alpha_{k k}^{+}}\right] \cong R_{k} / \mathfrak{q}_{k}^{\Delta} \overline{(\operatorname{cf} .}(4.5 .1)\right)$.

\section{LEMMA 4.11}

With assumptions as in Lemma 4.8, suppose further that one of the following equivalent conditions is satisfied (cf. (2.5.2), (2.3.6), Lemma 4.5):

$$
\begin{aligned}
& \text { Either } s_{k k}, z_{1 k k}, \ldots, z_{n k k} \text { form an }\left(R_{k} / \mathfrak{q}_{k}^{\Delta}\right) \text {-sequence, } \\
& \text { or } \theta_{k}, \zeta_{1 k k}, \ldots, \zeta_{n k k} \text { form an }\left(\tilde{R}_{k} / \tilde{\mathfrak{q}}_{k}^{+}\right) \text {-sequence. }
\end{aligned}
$$

Then

$$
\begin{gathered}
\pi_{j} \notin \bigcup \operatorname{Ass}\left(\tilde{R}_{k} / \tilde{\mathfrak{q}}_{k}\right) \quad \text { for every } j \leq k \text { or } s_{k k} \notin \bigcup \operatorname{Ass}\left(R_{k} / \mathfrak{q}_{k}^{\diamond}\right), \\
\bigcup_{\nu \geq 1} \operatorname{Ass}\left(\left(\tilde{R}_{k} / \tilde{\mathfrak{q}}_{k}^{+}\right) /\left(\theta_{k},\left(\mathfrak{q}_{k}^{-}\right)^{\nu}\right)\right) \cap R_{k}=\operatorname{Ass}\left(\tilde{R}_{k} /\left(\theta_{k}, \tilde{\mathfrak{q}}_{k}\right)\right) \cap R_{k} .
\end{gathered}
$$

\section{Proof}

First, we remark that (4.11.1) follows from the assumption and the facts that (cf. [8, Lemma 1.1])

$$
\mathfrak{q}_{k}^{\nabla}=\left(g_{j k k}^{-}-w_{j k}\right) \quad \text { and } \quad g_{j k k}^{-}-w_{j k} \equiv F_{j}\left(\underline{z_{k k}}\right) \quad\left(\bmod \mathfrak{q}_{k}^{\Delta}\right) .
$$

Then

$$
\begin{aligned}
& \bigcup_{\nu \geq 1} \operatorname{Ass}\left(\left(\tilde{R}_{k} / \tilde{\mathfrak{q}}_{k}^{+}\right) /\left(\theta_{k},\left(\mathfrak{q}_{k}^{-}\right)^{\nu}\right)\right) \cap R_{k} \\
& =\operatorname{Ass}\left(\left(\tilde{R}_{k} / \tilde{\mathfrak{q}}_{k}^{+}\right)\left[\underline{\tilde{\alpha}_{k k}^{-}}\right] / \theta_{k}\left(\tilde{R}_{k} / \tilde{\mathfrak{q}}_{k}^{+}\right)\left[\underline{\left.\tilde{\alpha}_{k k}^{-}\right]}\right) \cap R_{k}\right. \\
& =\operatorname{Ass}\left(\left(R_{k} / \mathfrak{q}_{k}^{\Delta}\right)\left[\underline{\tilde{\alpha}_{k k}^{-}}\right] / s_{k k}\left(R_{k} / \mathfrak{q}_{k}^{\Delta}\right)\left[\underline{\tilde{\alpha}_{k k}^{-}}\right]\right) \cap R_{k} \\
& =\bigcup_{\nu \geq 1} \operatorname{Ass}\left(R_{k} /\left(s_{k k}, \mathfrak{q}_{k}^{\Delta},\left(\mathfrak{q}_{k}^{\nabla}\right)^{\nu}\right)\right) \\
& =\operatorname{Ass}\left(R_{k} /\left(s_{k k}, \mathfrak{q}_{k}^{\Delta}, \mathfrak{q}_{k}^{\nabla}\right)\right) \\
& =\operatorname{Ass}\left(R_{k} /\left(s_{k k}, \mathfrak{q}_{k}^{\diamond}\right)\right) \\
& =\operatorname{Ass}\left(\tilde{R}_{k} /\left(\theta_{k}, \tilde{\mathfrak{q}}_{k}\right)\right) \cap R_{k},
\end{aligned}
$$


where $\tilde{\alpha}_{j k k}^{-}$is the image of $\alpha_{j k k}^{-}$in $Q\left(\tilde{R}_{k} / \tilde{\mathfrak{q}}_{k}^{+}\right)=Q\left(R_{k} / \mathfrak{q}_{k}^{\Delta}\right)$ (cf. (1.8.1), (4.5.1), (4.1.1), (4.11.1), (4.6.2)).

\section{LEMMA 4.12}

With notation and assumptions as in Lemma 4.11, we have

$$
\begin{array}{r}
\bigcup \operatorname{Ass}\left(\tilde{R}_{k} /\left(q_{k}, \tilde{\mathfrak{q}}_{k}^{-}\right)\right) \cap R_{k} \supset \bigcup \operatorname{Ass}\left(\tilde{R}_{k} /\left(q_{k}, \tilde{\mathfrak{q}}_{k}^{+}\right)\right) \cap R_{k}, \\
\bigcup\left\{\bigcup_{\nu \geq 1} \operatorname{Ass}\left(\left(\tilde{R}_{k} / \tilde{\mathfrak{q}}_{k}^{+}\right) /\left(\theta_{k},\left(\mathfrak{q}_{k}^{-}\right)^{\nu}\right)\right)\right\} \cap R_{k} \supset \bigcup \operatorname{Ass}\left(\tilde{R}_{k} /\left(\theta_{k}, \tilde{\mathfrak{q}}_{k}^{+}\right)\right) \cap R_{k} .
\end{array}
$$

\section{Proof}

We remark that, by (2.3.6) and (4.5.1), the first of the assumptions in Lemma 4.8 is equivalent to $q_{k}, \zeta_{1 k k}, \ldots, \zeta_{n k k}$ forming an $\left(\tilde{R}_{k} / \tilde{\mathfrak{q}}_{k}^{+}\right)$-sequence. The assertions above follow from Lemmas 4.11 and 1.11. Indeed, we have (cf. (4.10.1), (4.11.2))

$$
\begin{aligned}
& \bigcup \operatorname{Ass}\left(\tilde{R}_{k} /\left(q_{k}, \tilde{\mathfrak{q}}_{k}\right)\right) \cap R_{k} \supset \bigcup \operatorname{Ass}\left(\tilde{R}_{k} /\left(q_{k}, \tilde{\mathfrak{q}}_{k}^{+}\right)\right) \cap R_{k}, \\
& \bigcup \operatorname{Ass}\left(\tilde{R}_{k} /\left(\theta_{k}, \tilde{\mathfrak{q}}_{k}\right)\right) \cap R_{k} \supset \bigcup \operatorname{Ass}\left(\tilde{R}_{k} /\left(\theta_{k}, \tilde{\mathfrak{q}}_{k}^{+}\right)\right) \cap R_{k} .
\end{aligned}
$$

\section{LEMMA 4.13}

With notation and assumptions as in Lemma 4.11, take $a \in R_{k-2}$ or $a \in R_{k-1}$. If $\varepsilon_{k}$ is sufficiently large, then the following statements are equivalent to each other for every $i \leq k$ :

$$
\begin{aligned}
& a \in p_{i}^{e_{i}} R_{k-1}+\mathfrak{q}_{k-1}^{\Delta} \text { and } a \notin p_{i}^{e_{i}+1} R_{k-1}+\mathfrak{q}_{k-1}^{\Delta} ; \\
& a \in p_{i}^{e_{i}} \tilde{R}_{k-1}+\tilde{\mathfrak{q}}_{k-1}^{+} \text {and } a \notin p_{i}^{e_{i}+1} \tilde{R}_{k-1}+\tilde{\mathfrak{q}}_{k-1}^{+} ; \\
& a \in p_{i}^{e_{i}} \tilde{R}_{k}+\tilde{\mathfrak{q}}_{k} \text { and } a \text { is not contained in any primary component } \\
& \quad \text { of } p_{i}^{e_{i}+1} \tilde{R}_{k}+\tilde{\mathfrak{q}}_{k} ; \\
& a \in p_{i}^{e_{i}} \tilde{R}_{k}+\tilde{\mathfrak{q}}_{k} \text { and if we have } a \equiv p_{i}^{e_{i}} \beta \quad\left(\bmod \tilde{\mathfrak{q}}_{k}\right) \text { with } \beta \in \tilde{R}_{k}, \\
& \quad \text { then } \beta \notin \bigcup \operatorname{Ass}\left(\tilde{R}_{k} /\left(p_{i}, \tilde{\mathfrak{q}}_{k}\right)\right) ; \\
& a \in p_{i}^{e_{i}} \tilde{R}_{k}+\tilde{\mathfrak{q}}_{k}^{+} \text {and } a \notin p_{i}^{e_{i}+1} \tilde{R}_{k}+\tilde{\mathfrak{q}}_{k}^{+} ; \\
& a \in p_{i}^{e_{i}} R_{k}+\mathfrak{q}_{k}^{\Delta} \text { and } a \notin p_{i}^{e_{i}+1} R_{k}+\mathfrak{q}_{k}^{\Delta .}
\end{aligned}
$$

Proof

The equivalence of (4.13.1), (4.13.2), (4.13.5), and (4.13.6) follows from Lemma 4.5. That (4.13.3) implies (4.13.1) is a consequence of the following:

$$
\left(p_{i}^{e_{i}}, \tilde{\mathfrak{q}}_{k}\right) \cap R_{k-1}=\left(p_{i}^{e_{i}}, \mathfrak{q}_{k-1}^{\Delta}\right) \quad \text { and } \quad \mathfrak{Q} \cap R_{k-1}=\left(p_{i}^{e_{i}+1}, \mathfrak{q}_{k-1}^{\Delta}\right)
$$

for at least one primary component $\mathfrak{Q}$ of $\left(p_{i}^{e_{i}+1}, \tilde{\mathfrak{q}}_{k}\right)$, because $\theta_{k}$ is a non-zero divisor for $\tilde{R}_{k} /\left(p_{i}^{e_{i}+1}, \tilde{\mathfrak{q}}_{k}\right)$ and $\left(p_{i}^{e_{i}+1}, \mathfrak{q}_{k-1}^{\Delta}\right)$ is an irreducible ideal of $R_{k-1}$ (cf. Lemma 1.2). Because (4.13.4) clearly implies (4.13.3), to complete the proof, it 
suffices to show that (4.13.2) implies (4.13.4). Let

$$
a=p_{i}^{e_{i}} b+\sum_{j=1}^{r} c_{j} \alpha_{j(k-1)(k-1)}^{+}
$$

with $b, c_{j} \in \tilde{R}_{k-1}$ such that $b \notin\left(p_{i}, \tilde{\mathfrak{q}}_{k-1}^{+}\right)$. Then $a=p_{i}^{e_{i}}\left(b+q_{k} \kappa\right)+\sum_{j=1}^{r} d_{j} \alpha_{j k k}^{+}$ with $d_{j}, \kappa \in R_{k}\left[\underline{\alpha_{k k}^{+}}\right]$, because

$$
\alpha_{j(k-1)(k-1)}^{+}=\frac{q_{k}^{\nu_{k}}}{q_{k-1}^{\nu_{k-1}}} \alpha_{j k k}^{+}+\frac{q_{k}^{\varepsilon_{k}}}{q_{k-1}^{\nu_{k-1}}} \kappa_{j(k-1)(k-1)}^{+}
$$

with $\kappa_{j(k-1)(k-1)}^{+} \in R_{k}\left[\alpha_{(k-1)(k-1)}^{+}\right]$.

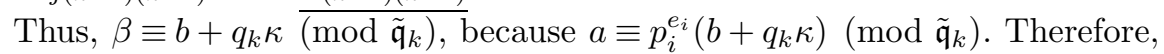
the assertion results from the following: if $b \in \tilde{R}_{k-1}$ and $b \notin p_{i} \tilde{R}_{k-1}+\tilde{\mathfrak{q}}_{k-1}^{+}$, then $b+q_{k} \kappa^{\prime}$ is a non-zero divisor for $\tilde{R}_{k} /\left(p_{i}, \tilde{\mathfrak{q}}_{k}\right)$ for every $\kappa^{\prime} \in \tilde{R}_{k}$ (cf. Lemma 1.2, (4.1.1)).

\section{LEMMA 4.14}

With assumptions as in Lemma 4.11, suppose that

$$
\pi_{1} \tilde{R}_{k-1}+\tilde{\mathfrak{q}}_{k-1}^{-}, \ldots, \pi_{k} \tilde{R}_{k-1}+\tilde{\mathfrak{q}}_{k-1}^{-} \text {are distinct prime ideals. }
$$

Assume further that, for every $j \leq k$,

$$
\begin{aligned}
& \pi_{j} \notin \bigcup \operatorname{Ass}\left(\left(\tilde{R}_{k-1} / \tilde{\mathfrak{q}}_{k-1}^{-}\right) / q_{k}\left(\tilde{R}_{k-1} / \tilde{\mathfrak{q}}_{k-1}^{-}\right)\right), \\
& \pi_{j} \notin \bigcup \operatorname{Ass}\left(\left(\tilde{R}_{k} / \tilde{\mathfrak{q}}_{k}^{-}\right) / q_{k}\left(\tilde{R}_{k} / \tilde{\mathfrak{q}}_{k}^{-}\right)\right) .
\end{aligned}
$$

Then $\pi_{1} \tilde{R}_{k}+\tilde{\mathfrak{q}}_{k}^{-}, \ldots, \pi_{k} \tilde{R}_{k}+\tilde{\mathfrak{q}}_{k}^{-}$are distinct prime ideals, and

$$
\left(\pi_{j}^{f_{j}} \tilde{R}_{k}+\tilde{\mathfrak{q}}_{k}^{-}\right) \cap \tilde{R}_{k-1}=\pi_{j}^{f_{j}} \tilde{R}_{k-1}+\tilde{\mathfrak{q}}_{k-1}^{-} \quad \text { whenever } \varepsilon_{k} \geq f_{j}
$$

\section{Proof}

By assumptions (4.14.1) and (4.14.2), we have

$$
q_{k} \notin \bigcup \operatorname{Ass}\left(\tilde{R}_{k-1} /\left(\pi_{j}, \tilde{\mathfrak{q}}_{k-1}^{-}\right)\right) \cup \bigcup \operatorname{Ass}\left(\tilde{R}_{k} /\left(\pi_{j}, \tilde{\mathfrak{q}}_{k}^{-}\right)\right) \quad \text { for each } j \leq k .
$$

Then $\left(\pi_{j}^{f_{j}}, \tilde{\mathfrak{q}}_{\ell}^{-}\right) \tilde{R}_{\ell}\left[1 / q_{\ell}\right] \cap \tilde{R}_{\ell}=\pi_{j}^{f_{j}} \tilde{R}_{\ell}+\tilde{\mathfrak{q}}_{\ell}^{-}$for $\ell=k-1$ and $k$. Moreover,

$$
\begin{aligned}
\left(\pi_{j}^{f_{j}}, \tilde{\mathfrak{q}}_{k}^{-}\right) \tilde{R}_{k}\left[1 / q_{k}\right] & =\left(\pi_{j}^{f_{j}}, \mathfrak{q}_{k}^{-}\right) \tilde{R}_{k}\left[1 / q_{k}\right] \\
& =\left(\pi_{j}^{f_{j}}, \mathfrak{q}_{k-1}^{-}\right) \tilde{R}_{k}\left[1 / q_{k}\right] \\
& =\left(\pi_{j}^{f_{j}}, \tilde{\mathfrak{q}}_{k-1}^{-}\right) \tilde{R}_{k}\left[1 / q_{k}\right]
\end{aligned}
$$

whenever $\varepsilon_{k} \geq f_{j}$ (cf. (2.3.14), (2.4.1), (4.2.2), (4.4.3)). This completes the proof. 


\section{LEMMA 4.15}

With assumptions as in Lemma 4.14, we have

$$
\begin{aligned}
\bigcap_{i=1}^{k}\left(p_{i}^{e_{i}}, \tilde{\mathfrak{q}}_{k}^{+}\right) \cap \bigcap_{j=1}^{k}\left(\pi_{j}^{f_{j}}, \tilde{\mathfrak{q}}_{k}^{-}\right)= & p_{1}^{e_{1}} \cdots p_{k}^{e_{k}} \pi_{1}^{f_{1}} \cdots \pi_{k}^{f_{k}} \tilde{R}_{k} \\
& +p_{1}^{e_{1}} \cdots p_{k}^{e_{k}} \tilde{\mathfrak{q}}_{k}^{-}+\pi_{1}^{f_{1}} \cdots \pi_{k}^{f_{k}} \tilde{\mathfrak{q}}_{k}^{+}+\tilde{\mathfrak{q}}_{k}^{+} \cap \tilde{\mathfrak{q}}_{k}^{-} .
\end{aligned}
$$

Proof

The assertion follows from Lemma 1.12. Indeed, by Lemmas 4.5 and 4.14, we get

$$
\bigcap_{i=1}^{k}\left(p_{i}^{e_{i}}, \tilde{\mathfrak{q}}_{k}^{+}\right)=p_{1}^{e_{1}} \cdots p_{k}^{e_{k}} \tilde{R}_{k}+\tilde{\mathfrak{q}}_{k}^{+} \quad \text { and } \quad \bigcap_{j=1}^{k}\left(\pi_{j}^{f_{j}}, \tilde{\mathfrak{q}}_{k}^{-}\right)=\pi_{1}^{f_{1}} \cdots \pi_{k}^{f_{k}} \tilde{R}_{k}+\tilde{\mathfrak{q}}_{k}^{-} \text {. }
$$

Moreover, $p_{1}^{e_{1}} \cdots p_{k}^{e_{k}}$ is a non-zero divisor on $\tilde{R}_{k} / \tilde{\mathfrak{q}}_{k}$ (cf. (4.6.2)) and $\pi_{1}^{f_{1}} \cdots \pi_{k}^{f_{k}}$ is a non-zero divisor for $\tilde{R}_{k} /\left(p_{1}^{e_{1}} \cdots p_{k}^{e_{k}}, \tilde{\mathfrak{q}}_{k}\right)$ (cf. (4.14.2), (4.10.2)).

\section{LEMMA 4.16}

With assumptions as in Lemma 4.14, take $a \in R_{k-1}$. Suppose that

$$
a \in \bigcap_{i=1}^{k}\left(p_{i}^{e_{i}}, \mathfrak{q}_{k-1}^{+}\right) \cap \bigcap_{j=1}^{k}\left(\pi_{j}^{f_{j}}, \tilde{\mathfrak{q}}_{k-1}^{-}\right) .
$$

If $\varepsilon_{k}, \nu_{k}$ are sufficiently large, then there exist $\pi \in R_{k}\left[q_{k} \underline{\alpha_{k k}^{+}}\right]$and a unit $u \in \tilde{R}_{k-1}$ such that

$$
a \equiv p_{1}^{e_{1}} \cdots p_{k}^{e_{k}} \cdot \pi_{1}^{f_{1}} \cdots \pi_{k}^{f_{k}} \cdot \pi \cdot u \quad\left(\bmod \tilde{\mathfrak{q}}_{k}^{-}\right) .
$$

Further, assume that

$$
a \notin \bigcup_{i=1}^{k}\left(p_{i}^{e_{i}+1}, \mathfrak{q}_{k-1}^{\Delta}\right) \cup \bigcup_{j=1}^{k}\left(\pi_{j}^{f_{j}+1}, \tilde{\mathfrak{q}}_{k-1}^{-}\right)
$$

Then

$$
\pi \notin \bigcup \operatorname{Ass}\left(\tilde{R}_{k} /\left(p_{i}, \tilde{\mathfrak{q}}_{k}^{+}\right)\right) \cup \bigcup \operatorname{Ass}\left(\tilde{R}_{k} /\left(\pi_{j}, \tilde{\mathfrak{q}}_{k}^{-}\right)\right)
$$

for every $i, j \leq k$. Moreover, when a is a prime element of $R_{k-1}$, the image of $\pi$ is either a prime element or a unit in $\tilde{R}_{k} / \tilde{\mathfrak{q}}_{k}^{-}$.

Proof

By the first assumption and by Lemma 4.15, we can express

$$
a=p_{1}^{e_{1}} \cdots p_{k}^{e_{k}} \cdot \pi_{1}^{f_{1}} \cdots \pi_{k}^{f_{k}} \cdot b+p_{1}^{e_{1}} \cdots p_{k}^{e_{k}} \cdot c+\pi_{1}^{f_{1}} \cdots \pi_{k}^{f_{k}} \cdot d+e
$$

with $b \in \tilde{R}_{k-1}, c \in \tilde{\mathfrak{q}}_{k-1}^{-}, d \in \tilde{\mathfrak{q}}_{k-1}^{+}$, and $e \in \tilde{\mathfrak{q}}_{k-1}^{+} \cap \tilde{\mathfrak{q}}_{k-1}^{-}$. That is,

$$
\begin{aligned}
c & =\sum c_{j}\left(\phi_{j(k-1)(k-1)}^{-}-w_{j(k-1)}\right), \\
d & =\sum d_{j} \alpha_{j(k-1)(k-1)}^{+},
\end{aligned}
$$




$$
\begin{aligned}
e=\sum e_{i j} \alpha_{i(k-1)(k-1)}^{+}( & \left.\phi_{j(k-1)(k-1)}^{-}-w_{j(k-1)}\right) \\
& +\sum e_{h}^{\prime} G_{h} \underline{\left(\underline{\zeta_{(k-1)(k-1)}}, \underline{\alpha_{(k-1)(k-1)}^{+}}\right)} .
\end{aligned}
$$

Then

$$
\begin{aligned}
c & =\sum c_{j}\left(\phi_{j k k}^{-}-w_{j k}\right)+\theta_{k}^{\varepsilon_{k}} \kappa_{k-1}^{-}, \\
d & =\sum d_{j}\left(\frac{q_{k}^{\nu_{k}}}{q_{k-1}^{\nu_{k-1}}} \alpha_{j k k}^{+}+\frac{q_{k}^{\varepsilon_{k}}}{q_{k-1}^{\nu_{k-1}}} \kappa_{j(k-1)(k-1)}^{+}\right) \\
& =\frac{q_{k}^{\nu_{k}}}{q_{k-1}^{\nu_{k-1}}} \sum d_{j} \alpha_{j k k}^{+}+\frac{q_{k}^{\varepsilon_{k}}}{q_{k-1}^{\nu_{k-1}}} \kappa_{k-1}^{+},
\end{aligned}
$$

and

$$
\begin{gathered}
e=\sum e_{i j}\left(\frac{q_{k}^{\nu_{k}}}{q_{k-1}^{\nu_{k-1}}} \alpha_{i k k}^{+}+\frac{q_{k}^{\varepsilon_{k}}}{q_{k-1}^{\nu_{k-1}}} \kappa_{i(k-1)(k-1)}^{+}\right)\left(\phi_{j k k}^{-}-w_{j k}+\theta_{k}^{\varepsilon_{k}} \kappa_{j(k-1)(k-1)}^{-}\right) \\
+\sum e_{h}^{\prime}\left\{\left(\frac{q_{k}^{\nu_{k}}}{q_{k-1}^{\nu_{k-1}}}\right)^{g_{h}}\left[G_{h}\left(\underline{\zeta_{k k}}, \underline{\alpha_{k k}^{+}}\right)+\theta_{k}^{\varepsilon_{k}} \delta_{h k(k-1)}^{+}\right]\right. \\
+\frac{q_{k}^{\varepsilon_{k}}}{q_{k-1}^{\nu_{k-1} g_{h}}}\left[\theta_{k}^{\varepsilon_{k}} \delta_{h(k-1)(k-1)}^{+}\right. \\
\left.\left.+\sum \lambda_{h j(k-1)(k-1)}^{+}\left(\phi_{j k k}^{-}-w_{j k}+\theta_{k}^{\varepsilon_{k}} \kappa_{j(k-1)(k-1)}^{-}\right)\right]\right\} \\
=\frac{q_{k}^{\nu_{k}}}{q_{k-1}^{\nu_{k-1}}} \sum e_{j}\left(\phi_{j k k}^{-}-w_{j k}\right) \\
+\left(\frac{q_{k}^{\nu_{k}}}{q_{k-1}^{\nu_{k-1}}}\right)^{g_{h}} \sum e_{h}^{\prime} G_{h}\left(\underline{\zeta_{k k}}, \underline{\alpha_{k k}^{+}}\right)+\frac{q_{k}^{\nu_{k}}}{q_{k-1}^{\nu_{k-1}}} \theta_{k}^{\varepsilon_{k}} \lambda_{k-1}^{+},
\end{gathered}
$$

where $\kappa_{k-1}^{-} \in \tilde{R}_{k-1}, \kappa_{k-1}^{+} \in \tilde{R}_{k-1}\left[\underline{a_{k}}, \underline{c_{k}}\right], e_{j} \in \tilde{R}_{k-1}\left[\underline{a_{k}}, \underline{c_{k}}, \underline{\alpha_{k k}^{+}}\right]$, and $\lambda_{k-1}^{+} \in$ $\tilde{R}_{k-1}\left[\underline{a_{k}}, \underline{b_{k}}, \underline{\alpha_{k k}^{+}}\right]$(cf. (2.4.2), (3.3.1), (3.3.2)).

Thus,

$$
\begin{aligned}
a= & p_{1}^{e_{1}} \cdots p_{k}^{e_{k}} \cdot \pi_{1}^{f_{1}} \cdots \pi_{k}^{f_{k}} \\
& \times\left[b+\frac{\theta_{k}^{\varepsilon_{k}}}{\pi_{1}^{f_{1}} \cdots \pi_{k}^{f_{k}}} \kappa_{k-1}^{-}+\frac{q_{k}^{\nu_{k}}}{p_{1}^{e_{1}} \cdots p_{k}^{e_{k}} \cdot q_{k-1}^{\nu_{k}-1}} \sum d_{j} \alpha_{j k k}^{+}\right. \\
& \left.\quad+\frac{q_{k}^{\varepsilon_{k}}}{p_{1}^{e_{1}} \cdots p_{k}^{e_{k}} \cdot q_{k-1}^{\nu_{k-1}}} \kappa_{k-1}^{+}+\frac{q_{k}^{\nu_{k}} \theta_{k}^{\varepsilon_{k}}}{p_{1}^{e_{1}} \cdots p_{k}^{e_{k}} \cdot \pi_{1}^{f_{1}} \cdots \pi_{k}^{f_{k}} \cdot q_{k-1}^{\nu_{k-1}}} \lambda_{k-1}^{+}\right] \\
+ & p_{1}^{e_{1}} \cdots p_{k}^{e_{k}} \sum c_{j}\left(\phi_{j k k}^{-}-w_{j k}\right) \\
+ & +\frac{q_{k}^{\nu_{k}}}{q_{k-1}^{\nu_{k-1}}} \sum e_{j}\left(\phi_{j k k}^{-}-w_{j k}\right)+\left(\frac{q_{k}^{\nu_{k}}}{q_{k-1}^{\nu_{k-1}}}\right)^{g_{h}} \sum e_{h}^{\prime} G_{h}\left(\underline{\zeta_{k k}}, \underline{\alpha_{k k}^{+}}\right) .
\end{aligned}
$$


Now put

$$
\begin{aligned}
\pi^{\prime}=b+ & \frac{\theta_{k}^{\varepsilon_{k}}}{\pi_{1}^{f_{1}} \cdots \pi_{k}^{f_{k}}} \kappa_{k-1}^{-} \\
+ & \frac{q_{k}^{\nu_{k}}}{p_{1}^{e_{1}} \cdots p_{k}^{e_{k}} \cdot q_{k-1}^{\nu_{k-1}}} \sum d_{j} \alpha_{j k k}^{+}+\frac{q_{k}^{\varepsilon_{k}}}{p_{1}^{e_{1}} \cdots p_{k}^{e_{k}} \cdot q_{k-1}^{\nu_{k-1}}} \kappa_{k-1}^{+} \\
& \quad+\frac{q_{k}^{\nu_{k}} \theta_{k}^{\varepsilon_{k}}}{p_{1}^{e_{1}} \cdots p_{k}^{e_{k}} \cdot \pi_{1}^{f_{1}} \cdots \pi_{k}^{f_{k}} \cdot q_{k-1}^{\nu_{k-1}}} \lambda_{k-1}^{+} .
\end{aligned}
$$

Then there exists $w \in R_{k-1}\left[\underline{\alpha_{(k-1)(k-1)}^{+}}\right] \backslash \mathfrak{N}_{k-1}$ such that $\pi=w \pi^{\prime} \in R_{k}\left[q_{k} \underline{\alpha_{k k}^{+}}\right]$. Hence, by letting $u=1 / w$, we get (4.16.2).

Assume further that $a$ satisfies (4.16.3). Then by Lemma 4.13, we see that

$$
\pi \notin \bigcup \operatorname{Ass}\left(\tilde{R}_{k} /\left(p_{i}, \tilde{\mathfrak{q}}_{k}\right)\right) \quad \text { for each } i \leq k .
$$

Thus, $\pi$ satisfies (4.16.4) by Lemmas 4.10 and 4.14. Finally, when $a$ is a prime element of $R_{k-1}$, the image of $\pi$ in $\tilde{R}_{k} / \tilde{\mathfrak{q}}_{k}^{-}$is either a prime element or a unit, because

$$
\begin{aligned}
\left(\tilde{R}_{k} /\left(\pi, \tilde{\mathfrak{q}}_{k}^{-}\right)\right)\left[\frac{1}{q_{k} \theta_{k}}\right] & \cong\left(\tilde{R}_{k} /\left(a, \tilde{\mathfrak{q}}_{k}^{-}\right)\right)\left[\frac{1}{q_{k} \theta_{k}}\right] \\
& =\left(\left(R_{k-1} / a R_{k-1}\right)\left[\frac{1}{q_{k} \theta_{k}}\right]\left[\underline{a_{k}}, \underline{b_{k}}, \underline{c_{k}}\right] / \mathfrak{q}_{k}^{-}\right)_{U_{k}} \\
& \cong\left(\left(R_{k-1} / a R_{k-1}\right)\left[\underline{a_{k}}, \underline{b_{k}}\right]\right)_{U_{k}^{\prime}},
\end{aligned}
$$

where $U_{k}$ and $U_{k}^{\prime}$ are multiplicative sets.

\section{LEMMA 4.17}

With notation and assumptions as in Lemma 4.16, take $a \in R_{k-2}$. Assume that

$$
a \equiv p_{1}^{e_{1}} \cdots p_{k}^{e_{k}} \cdot \pi_{1}^{f_{1}} \cdots \pi_{k}^{f_{k}} \cdot \epsilon \quad\left(\bmod \tilde{\mathfrak{q}}_{k-1}^{-}\right)
$$

with $\epsilon \in \tilde{R}_{k-1}$ such that the image of $\epsilon$ in $\tilde{R}_{k-1} / \tilde{\mathfrak{q}}_{k-1}^{-}$is a unit. Then, for sufficiently large $\varepsilon_{k}$, we have

$$
a \equiv p_{1}^{e_{1}} \cdots p_{k}^{e_{k}} \cdot \pi_{1}^{f_{1}} \cdots \pi_{k}^{f_{k}} \cdot \epsilon^{\prime} \quad\left(\bmod \tilde{\mathfrak{q}}_{k}^{-}\right)
$$

where $\epsilon^{\prime} \in \tilde{R}_{k}$ and the image of $\epsilon^{\prime}$ in $\tilde{R}_{k} / \tilde{\mathfrak{q}}_{k}^{-}$is a unit.

Proof

By assumption, we can write

$$
a=p_{1}^{e_{1}} \cdots p_{k}^{e_{k}} \cdot \pi_{1}^{f_{1}} \cdots \pi_{k}^{f_{k}} \cdot b+p_{1}^{e_{1}} \cdots p_{k}^{e_{k}} \cdot c+\pi_{1}^{f_{1}} \cdots \pi_{k}^{f_{k}} \cdot d+e
$$

with $b \in \tilde{R}_{k-1}, c \in \tilde{\mathfrak{q}}_{k-1}^{-}, d \in \tilde{\mathfrak{q}}_{k-1}^{+}, e \in \tilde{\mathfrak{q}}_{k-1}^{+} \cap \tilde{\mathfrak{q}}_{k-1}^{-}$(cf. Lemmas 4.13, 4.16). Hence, using the same notation as in the proof of Lemma 4.16, we get

$$
a \equiv p_{1}^{e_{1}} \cdots p_{k}^{e_{k}} \cdot \pi_{1}^{f_{1}} \cdots \pi_{k}^{f_{k}} \cdot \pi^{\prime} \quad\left(\bmod \tilde{\mathfrak{q}}_{k}^{-}\right) .
$$


Incidentally, $b \equiv \epsilon\left(\bmod \tilde{\mathfrak{q}}_{k-1}\right)$. Indeed, $q_{k} \theta_{k}$ is a non-zero divisor for $\tilde{R}_{k-1} / \tilde{\mathfrak{q}}_{k-1}$ and

$$
a \equiv p_{1}^{e_{1}} \cdots p_{k}^{e_{k}} \cdot \pi_{1}^{f_{1}} \cdots \pi_{k}^{f_{k}} \cdot b \quad\left(\bmod \tilde{\mathfrak{q}}_{k-1}\right) .
$$

Thus, the image of $b$ in $\tilde{R}_{k-1} / \tilde{\mathfrak{q}}_{k-1}$ is a unit; that is, $b \notin \tilde{\mathfrak{n}}_{k-1}$. Consequently, $\pi^{\prime} \notin$ $\tilde{\mathfrak{n}}_{k}$. Therefore, the image of $\epsilon^{\prime}$ in $\tilde{R}_{k} / \tilde{\mathfrak{q}}_{k}^{-}$is a unit, because $\epsilon^{\prime} \equiv \pi^{\prime}\left(\bmod \tilde{\mathfrak{q}}_{k}^{-}\right)$.

\section{Factorial numbering}

\subsection{Simple numbering on $\mathcal{P}$}

With notation as in Section 2, because $R_{k}=R_{k-1}\left(\underline{a_{k}}, \underline{b_{k}}, \underline{c_{k}}\right)$ and $R=\bigcup_{k} R_{k}$ (cf. (2.1.1)), each height-one prime ideal $\mathfrak{p}$ of $R$ is generated by a unique prime element (up to a unit multiple) $\rho \in S_{k}$. Thus, choosing such uniquely determined $\rho \in S_{k}$ for each height-one prime ideal $\mathfrak{p}$ of $R$, we get a set of prime elements that represents the height-one prime ideals of $R$, and we denote the set by $\mathcal{P}$ (cf. (2.1.2)). That is,

$$
\mathcal{P} \subset \mathfrak{N} \backslash\{0\},
$$

which contains, for each height-one prime ideal $\mathfrak{p} \in \operatorname{Spec}(R)$, exactly one $\rho \in S_{k}$ such that $\rho R=\mathfrak{p}$. Then, $\mathcal{P}$ is a countable set and we may assume that it contains $z_{0}$ and an infinite number of elements of $S_{0}$.

On the other hand, with notation as in Section 4, let

$$
\tilde{R}_{h-1}(\boldsymbol{a}, \boldsymbol{b}, \boldsymbol{c}):=\tilde{R}_{h-1}\left(a_{i k}, b_{i k}, c_{j k}\right)
$$

where $i=1, \ldots, n$ and $j=1, \ldots, r$ with $k=h, h+1, \ldots$, and let

$$
\tilde{R}_{h-1}(\boldsymbol{a}, \boldsymbol{b}, \boldsymbol{c})_{\leq \ell}:=\tilde{R}_{h-1}\left(a_{i \tau}, b_{i \tau}, c_{j \tau}\right)
$$

where $i=1, \ldots, n$ and $j=1, \ldots, r$ with $\tau=h, \ldots, \ell$ (i.e., $\tau \leq \ell$ ).

Now, before giving factorial numbering, we fix a simple numbering $\rho_{1}, \rho_{2}, \ldots$, $\rho_{h}, \ldots$ to the members of $\mathcal{P}$ as follows:

$$
\rho_{1}=z_{0} \quad \text { and } \quad \rho_{h} \in R_{h-2} \quad \text { for } h \geq 2 .
$$

\section{PROPOSITION 5.2 (FACTORIAL NUMBERING)}

With notation as in Section 2 and with simple numbering (5.1.4), we can choose two sequences of elements of $Q(R)$, namely, $\pi_{1}, \pi_{2}, \ldots, \pi_{j}, \ldots$ and $p_{1}, p_{2}, \ldots, p_{i}, \ldots$, such that

$$
\begin{aligned}
& \pi_{1}:=\rho_{1}\left(=z_{0}\right) \quad \text { and } \quad \pi_{j} \in R_{j-1}\left[q_{j-1} \underline{\alpha_{(j-1)(j-1)}^{+}}\right] \quad \text { for } j \geq 2, \\
& p_{1} \in \mathcal{P} \cap R_{0} \quad \text { and } \quad p_{i} \in \mathcal{P} \cap R_{i-2} \quad \text { for } i \geq 2,
\end{aligned}
$$

and for each natural number $k$, they satisfy the following:

$$
q_{k}, \theta_{k} \text { form an }\left(\tilde{R}_{k-1} / \tilde{\mathfrak{q}}_{k-1}\right) \text {-sequence; }
$$$$
q_{k}, \theta_{k} \text { form an }\left(\tilde{R}_{k} / \tilde{\mathfrak{q}}_{k}\right) \text {-sequence; }
$$

$$
p_{1} \tilde{R}_{k-1}+\tilde{\mathfrak{q}}_{k-1}^{+}, \ldots, p_{k} \tilde{R}_{k-1}+\tilde{\mathfrak{q}}_{k-1}^{+} \text {and } \pi_{1} \tilde{R}_{k-1}+\tilde{\mathfrak{q}}_{k-1}^{-}, \ldots,
$$


$\pi_{k} \tilde{R}_{k-1}+\tilde{\mathfrak{q}}_{k-1}^{-}$are mutually distinct prime ideals of $\tilde{R}_{k-1}$; $q_{k}, z_{1 k k}, \ldots, z_{n k k}$ form an $\left(R_{k} / \mathfrak{q}_{k}^{\Delta}\right)$-sequence; $s_{k k} z_{1 k k}, \ldots, z_{n k k}$ form an $\left(R_{k} / \mathfrak{q}_{k}^{\Delta}\right)$-sequence; $q_{k}, z_{1 k k}, \ldots, z_{n k k}$ form an $\left(R_{k} / \mathfrak{q}_{k}^{\nabla}\right)$-sequence.

$W e$ can take these elements so that each $\rho_{h} \in \mathcal{P}$ is decomposed as follows:

$$
\rho_{h} \equiv p_{1}^{e_{h 1}} \cdots p_{2 h-3}^{e_{h(2 h-3)}} \cdot \pi_{1}^{f_{h 1}} \cdots \pi_{2 h-3}^{f_{h(2 h-3)}} \cdot \pi_{2 h-2}^{f_{h(2 h-2)}} \cdot u_{h k} \quad\left(\bmod \tilde{\mathfrak{q}}_{k}^{-}\right),
$$

where $u_{h k} \in \tilde{R}_{k} \backslash \tilde{\mathfrak{n}}_{k}$ for $k \geq 2 h-3$ (cf. (4.16.2), (4.17.1)).

On the other hand, we can find natural numbers $\mu_{k}, \nu_{k}$, and $\varepsilon_{k}$ such that

$$
\begin{aligned}
& \mu_{1}<\mu_{2}<\cdots<\mu_{k}<\mu_{k+1}<\cdots \\
& \wedge \wedge \wedge \wedge \wedge \\
& \nu_{1}<\nu_{2}<\cdots<\nu_{k}<\nu_{k+1}<\cdots \\
& \wedge \wedge \wedge \wedge \wedge \\
& \varepsilon_{1}<\varepsilon_{2}<\cdots<\varepsilon_{k}<\varepsilon_{k+1}<\cdots
\end{aligned}
$$

and they satisfy

$$
q_{k}^{\varepsilon_{k}} \theta_{k} \in R_{k-1} \quad \text { and } \quad \nu_{2 k}>N_{2 k}:=\max \left\{e_{h i}+\nu_{2 k-1}, f_{h j}, f_{h(2 h-2)}\right\}
$$

for $1 \leq i, j \leq 2 h-3 ; 1 \leq h \leq k+1$ (cf. (4.5.2), (4.14.3), (4.16.2)). Finally, we can give enumerations on

$$
\begin{aligned}
& \mathcal{X}_{i}^{+}:=\text {the set of height-one prime ideals of } \tilde{R}_{i-1}(\boldsymbol{a}, \boldsymbol{b}, \boldsymbol{c}) /\left(p_{i}, \tilde{\mathfrak{q}}_{i-1}^{+}\right), \\
& \mathcal{X}_{j}^{-}:=\text {the set of height-one prime ideals of } \tilde{R}_{j-1}(\boldsymbol{a}, \boldsymbol{b}, \boldsymbol{c}) /\left(\pi_{j}, \tilde{\mathfrak{q}}_{j-1}^{-}\right) .
\end{aligned}
$$

Namely, we have (cf. (5.1.2), (5.1.3))

$$
\begin{aligned}
& \mathcal{X}_{i}^{+}:=\left\{\tilde{\mathfrak{p}}_{i h}^{+}:=\tilde{\mathfrak{P}}_{i h}^{+} /\left(p_{i}, \tilde{\mathfrak{q}}_{i-1}^{+}\right)\right\} \text {such that } \tilde{\mathfrak{P}}_{i h}^{+} \subset \tilde{R}_{i-1}(\boldsymbol{a}, \boldsymbol{b}, \boldsymbol{c})_{\leq h-2}, \\
& \mathcal{X}_{j}^{-}:=\left\{\tilde{\mathfrak{p}}_{j h}^{-}:=\tilde{\mathfrak{P}}_{j h}^{-} /\left(\pi_{j}, \tilde{\mathfrak{q}}_{j-1}^{-}\right)\right\} \text {such that } \tilde{\mathfrak{P}}_{j h}^{-} \subset \tilde{R}_{j-1}(\boldsymbol{a}, \boldsymbol{b}, \boldsymbol{c})_{\leq h-2} .
\end{aligned}
$$

That is, because $\mathcal{X}_{i}^{+}$and $\mathcal{X}_{j}^{-}$are defined to be the set of height-one prime ideals of $\tilde{R}_{i-1}(\boldsymbol{a}, \boldsymbol{b}, \boldsymbol{c}) /\left(p_{i}, \tilde{\mathfrak{q}}_{i-1}^{+}\right)$and of $\tilde{R}_{j-1}(\boldsymbol{a}, \boldsymbol{b}, \boldsymbol{c}) /\left(\pi_{j}, \tilde{\mathfrak{q}}_{j-1}^{-}\right), \tilde{\mathfrak{P}}_{i h}^{+}$should be an ideal of $\tilde{R}_{i-1}(\boldsymbol{a}, \boldsymbol{b}, \boldsymbol{c})$ generated by the elements of $\tilde{R}_{i-1}(\boldsymbol{a}, \boldsymbol{b}, \boldsymbol{c})_{\leq h-2}$ and $\tilde{\mathfrak{P}}_{j h}^{-}$should be an ideal of $\tilde{R}_{j-1}(\boldsymbol{a}, \boldsymbol{b}, \boldsymbol{c})$ generated by the elements of $\tilde{R}_{j-1}(\boldsymbol{a}, \boldsymbol{b}, \boldsymbol{c})_{\leq h-2}$.

Arranging the members of $\mathcal{X}_{1}^{+}, \mathcal{X}_{2}^{+}, \ldots, \mathcal{X}_{i}^{+}, \ldots$ and those of $\mathcal{X}_{1}^{-}, \mathcal{X}_{2}^{-}, \ldots$, $\mathcal{X}_{j}^{-}, \ldots$ in the following manner:

$$
\begin{array}{cccccc}
\tilde{\mathfrak{p}}_{11}^{ \pm} & \tilde{\mathfrak{p}}_{12}^{ \pm} & \tilde{\mathfrak{p}}_{13}^{ \pm} & \ldots & \tilde{\mathfrak{p}}_{1 h}^{ \pm} & \ldots \\
\tilde{\mathfrak{p}}_{21}^{ \pm} & \tilde{\mathfrak{p}}_{22}^{ \pm} & \ldots & \ldots & \ldots & \ldots \\
\ldots & \ldots & \ldots & \ldots & \ldots & \ldots \\
\ldots & \ldots & \ldots & \ldots & \ldots & \ldots \\
\tilde{\mathfrak{p}}_{\ell 1}^{ \pm} & \tilde{\mathfrak{p}}_{\ell 2}^{ \pm} & \ldots & \ldots & \tilde{\mathfrak{p}}_{\ell h}^{ \pm} & \ldots \\
\ldots & \ldots & \ldots & \ldots & \ldots & \ldots \\
\ldots & \ldots & \ldots & \ldots & \ldots & \ldots
\end{array}
$$


we get numberings on $\mathcal{X}^{+}:=\bigcup \mathcal{X}_{i}^{+}$and on $\mathcal{X}^{-}:=\bigcup \mathcal{X}_{j}^{-}$, specifically,

$$
\tilde{\mathfrak{p}}_{11}^{+}, \tilde{\mathfrak{p}}_{12}^{+}, \tilde{\mathfrak{p}}_{21}^{+}, \tilde{\mathfrak{p}}_{13}^{+}, \tilde{\mathfrak{p}}_{22}^{+}, \tilde{\mathfrak{p}}_{31}^{+}, \ldots \quad \text { and } \quad \tilde{\mathfrak{p}}_{11}^{-}, \tilde{\mathfrak{p}}_{12}^{-}, \tilde{\mathfrak{p}}_{21}^{-}, \tilde{\mathfrak{p}}_{13}^{-}, \tilde{\mathfrak{p}}_{22}^{-}, \tilde{\mathfrak{p}}_{31}^{-}, \ldots
$$

Further, if $\tilde{\mathfrak{p}}_{\ell h}^{-}$(or $\left.\tilde{\mathfrak{p}}_{\ell h}^{+}\right)$is the $k$ th member in $\mathcal{X}^{-}$(or in $\left.\mathcal{X}^{+}\right)$, then we can take $\pi_{j}, p_{i}$ such that

$$
\pi_{2 k+1} \in \tilde{\mathfrak{P}}_{\ell h}^{+} \quad \text { and } \quad p_{2 k+1} \in \tilde{\mathfrak{P}}_{\ell h}^{-}
$$

In the rest of this section, we carry out the proof of Proposition 5.2 by induction on $k$.

\subsection{Choice of $\pi_{1}$ and $p_{1}$ and the case $k=1$}

Let $\pi_{1}=z_{0}$. Then $\pi_{1} \notin \bigcup \operatorname{Ass}\left(\tilde{R}_{0} / \tilde{\mathfrak{q}}_{0}\right)$ and $\pi_{1} \tilde{R}_{0}+\tilde{\mathfrak{q}}_{0}^{-}$is a prime ideal of $\tilde{R}_{0}$. Next take $p_{1} \in \mathcal{P} \cap R_{0}$ such that $p_{1} \notin \bigcup \operatorname{Ass}\left(\tilde{R}_{0} /\left(\pi_{1}, \tilde{\mathfrak{q}}_{0}\right)\right)$ and such that $p_{1}, x_{1}, \ldots, x_{n}$, $w_{1}, \ldots, w_{r}, p_{1}, y_{1}, \ldots, y_{n}, w_{1}, \ldots, w_{r}$, and $p_{1}, z_{1}, \ldots, z_{n}, w_{1}, \ldots, w_{r}$, respectively, form part of the regular systems of parameters of $R$. Then

- $\pi_{1} \notin \bigcup \operatorname{Ass}\left(\tilde{R}_{0} / \tilde{\mathfrak{q}}_{0}\right)$ and $\pi_{1} \tilde{R}_{0}+\tilde{\mathfrak{q}}_{0}^{-}$is a prime ideal of $\tilde{R}_{0}$,

- $q_{1}, \theta_{1}$ form an $\left(\tilde{R}_{0} / \tilde{\mathfrak{q}}_{0}\right)$-sequence,

- $q_{1}, \theta_{1}$ form an $\left(\tilde{R}_{1} / \tilde{\mathfrak{q}}_{1}\right)$-sequence,

- $p_{1} \tilde{R}_{0}+\tilde{\mathfrak{q}}_{0}^{+}$and $\pi_{1} \tilde{R}_{0}+\tilde{\mathfrak{q}}_{0}^{-}$are mutually distinct prime ideals of $\tilde{R}_{0}$,

- $q_{1}, z_{111}, \ldots, z_{n 11}$ form an $\left(R_{1} / \mathfrak{q}_{1}^{\Delta}\right)$-sequence,

- $s_{11}, z_{111}, \ldots, z_{n 11}$ form an $\left(R_{1} / \mathfrak{q}_{1}^{\Delta}\right)$-sequence,

- $q_{1}, z_{111}, \ldots, z_{n 11}$ form an $\left(R_{1} / \mathfrak{q}_{1}^{\nabla}\right)$-sequence.

Finally, we can give enumerations on the sets of height-one prime ideals

$$
\mathcal{X}_{1}^{+} \text {of } \tilde{R}_{0}(\boldsymbol{a}, \boldsymbol{b}, \boldsymbol{c}) /\left(p_{1}, \tilde{\mathfrak{q}}_{0}^{+}\right) \quad \text { and } \quad \mathcal{X}_{1}^{-} \text {of } \tilde{R}_{0}(\boldsymbol{a}, \boldsymbol{b}, \boldsymbol{c}) /\left(\pi_{1}, \tilde{\mathfrak{q}}_{0}^{-}\right) .
$$

Namely,

$$
\mathcal{X}_{1}^{+}:=\left\{\tilde{\mathfrak{p}}_{1 h}^{+}:=\tilde{\mathfrak{P}}_{1 h}^{+} /\left(p_{1}, \tilde{\mathfrak{q}}_{0}^{+}\right)\right\} \quad \text { and } \quad \mathcal{X}_{1}^{-}:=\left\{\tilde{\mathfrak{p}}_{1 h}^{-}:=\tilde{\mathfrak{P}}_{1 h}^{-} /\left(\pi_{1}, \tilde{\mathfrak{q}}_{0}^{-}\right)\right\}
$$

such that $\tilde{\mathfrak{P}}_{1 h}^{+} \subset \tilde{R}_{0}(\boldsymbol{a}, \boldsymbol{b}, \boldsymbol{c})_{\leq h-2}$ and $\tilde{\mathfrak{P}}_{1 h}^{-} \subset \tilde{R}_{0}(\boldsymbol{a}, \boldsymbol{b}, \boldsymbol{c})_{\leq h-2}$.

5.4. Inductive assumptions up to $k=2 \kappa-1$

For a natural number $\kappa$, we may assume by induction that $\pi_{1}, \ldots, \pi_{2 \kappa-1}$ and $p_{1}, \ldots, p_{2 \kappa-1}$ have been chosen as follows:

$$
\pi_{j} \in R_{j-1}\left[q_{j-1} \underline{\alpha_{(j-1)(j-1)}^{+}}\right] \quad \text { and } \quad p_{i} \in \mathcal{P} \cap R_{i-2} \quad \text { for } i, j \leq 2 \kappa-1,
$$

and

- $q_{m}, \theta_{m}$ form an $\left(\tilde{R}_{m-1} / \tilde{\mathfrak{q}}_{m-1}\right)$-sequence,

- $q_{m}, \theta_{m}$ form an $\left(\tilde{R}_{m} / \tilde{\mathfrak{q}}_{m}\right)$-sequence,

- $p_{1} \tilde{R}_{m-1}+\tilde{\mathfrak{q}}_{m-1}^{+}, \ldots, p_{m} \tilde{R}_{m-1}+\tilde{\mathfrak{q}}_{m-1}^{+}$and $\pi_{1} \tilde{R}_{m-1}+\tilde{\mathfrak{q}}_{m-1}^{-}, \ldots$, $\pi_{m} \tilde{R}_{m-1}+\tilde{\mathfrak{q}}_{m-1}^{-}$are mutually distinct prime ideals of $\tilde{R}_{m-1}$,

- $q_{m}, z_{1 m m}, \ldots, z_{n m m}$ form an $\left(R_{m} / \mathfrak{q}_{m}^{\Delta}\right)$-sequence,

- $s_{m m}, z_{1 m m}, \ldots, z_{n m m}$ form an $\left(R_{m} / \mathfrak{q}_{m}^{\Delta}\right)$-sequence,

- $q_{m}, z_{1 m m}, \ldots, z_{n m m}$ form an $\left(R_{m} / \mathfrak{q}_{m}^{\nabla}\right)$-sequence 
for $m \leq 2 \kappa-1$. Further, we may assume that, for $2 h-2 \leq 2 \kappa-1$, each $\rho_{h} \in \mathcal{P}$ is decomposed by the elements above as follows:

$$
\rho_{h} \equiv p_{1}^{e_{h 1}} \cdots p_{2 h-3}^{e_{h(2 h-3)}} \cdot \pi_{1}^{f_{h 1}} \cdots \pi_{2 h-3}^{f_{h(2 h-3)}} \cdot \pi_{2 h-2}^{f_{h(2 h-2)}} \cdot u_{h m} \quad\left(\bmod \tilde{\mathfrak{q}}_{m}^{-}\right),
$$

where $u_{h m} \in \tilde{R}_{m} \backslash \tilde{\mathfrak{n}}_{m}$ with $2 h-3 \leq m \leq 2 \kappa-1$ (cf. (4.16.2), (4.17.1)). On the other hand, we may assume that we find natural numbers $\mu_{k}, \nu_{k}$, and $\varepsilon_{k}$ such that

$$
\begin{aligned}
& \mu_{1}<\mu_{2}<\cdots<\mu_{m}<\cdots<\mu_{2 \kappa-1} \\
& \wedge \wedge \wedge \wedge \wedge \\
& \nu_{1}<\nu_{2}<\cdots<\nu_{m}<\cdots<\nu_{2 \kappa-1} \\
& \wedge \wedge \wedge \wedge \wedge \\
& \varepsilon_{1}<\varepsilon_{2}<\cdots<\varepsilon_{m}<\cdots<\varepsilon_{2 \kappa-1}
\end{aligned}
$$

that satisfy

$$
q_{m}^{\varepsilon_{m}} \theta_{m} \in R_{m-1} \text { and } \nu_{2 \ell}>N_{2 \ell}:=\max \left\{e_{h i}+\nu_{2 \ell-1}, f_{h j}, f_{h(2 h-2)}\right\}
$$

for $m \leq 2 \kappa-1, i, j \leq 2 h-3$, and $h \leq \ell+1 \leq \kappa$ (cf. (4.5.2), (4.14.3), (4.16.2)).

Finally, we may assume that, for $i, j \leq 2 \kappa-1$, we can give enumerations

$$
\begin{aligned}
& \mathcal{X}_{i}^{+}:=\text {the set of height-one prime ideals of } \tilde{R}_{i-1}(\boldsymbol{a}, \boldsymbol{b}, \boldsymbol{c}) /\left(p_{i}, \tilde{\mathfrak{q}}_{i-1}^{+}\right), \\
& \mathcal{X}_{j}^{-}:=\text {the set of height-one prime ideals of } \tilde{R}_{j-1}(\boldsymbol{a}, \boldsymbol{b}, \boldsymbol{c}) /\left(\pi_{j}, \tilde{\mathfrak{q}}_{j-1}^{-}\right) .
\end{aligned}
$$

Namely (cf. (5.1.2), (5.1.3)),

$$
\begin{aligned}
\mathcal{X}_{i}^{+} & :=\left\{\tilde{\mathfrak{p}}_{i h}^{+}:=\tilde{\mathfrak{P}}_{i h}^{+} /\left(p_{i}, \tilde{\mathfrak{q}}_{i-1}^{+}\right)\right\} \text {such that } \tilde{\mathfrak{P}}_{i h}^{+} \subset \tilde{R}_{i-1}(\boldsymbol{a}, \boldsymbol{b}, \boldsymbol{c})_{\leq h-2}, \\
\mathcal{X}_{j}^{-}: & :=\left\{\tilde{\mathfrak{p}}_{j h}^{-}:=\tilde{\mathfrak{P}}_{j h}^{-} /\left(\pi_{j}, \tilde{\mathfrak{q}}_{j-1}^{-}\right)\right\} \text {such that } \tilde{\mathfrak{P}}_{j h}^{-} \subset \tilde{R}_{j-1}(\boldsymbol{a}, \boldsymbol{b}, \boldsymbol{c})_{\leq h-2} .
\end{aligned}
$$

Arranging the members of $\mathcal{X}_{1}^{+}, \mathcal{X}_{2}^{+}, \ldots, \mathcal{X}_{i}^{+}, \ldots$ and those of $\mathcal{X}_{1}^{-}, \mathcal{X}_{2}^{-}, \ldots$, $\mathcal{X}_{j}^{-}, \ldots$ in the following manner:

$$
\begin{array}{llllll}
\tilde{\mathfrak{p}}_{11}^{ \pm} & \tilde{\mathfrak{p}}_{12}^{ \pm} & \tilde{\mathfrak{p}}_{13}^{ \pm} & \ldots & \tilde{\mathfrak{p}}_{1 h}^{ \pm} & \ldots \\
\tilde{\mathfrak{p}}_{21}^{ \pm} & \tilde{\mathfrak{p}}_{22}^{ \pm} & \ldots & \ldots & \ldots & \ldots \\
\ldots & \ldots & \ldots & \ldots & \ldots & \ldots \\
\ldots & \ldots & \ldots & \ldots & \ldots & \ldots \\
\tilde{\mathfrak{p}}_{\ell 1}^{ \pm} & \tilde{\mathfrak{p}}_{\ell 2}^{ \pm} & \ldots & \ldots & \tilde{\mathfrak{p}}_{\ell h}^{ \pm} & \ldots \\
\ldots & \ldots & \ldots & \ldots & \ldots & \ldots \\
\tilde{\mathfrak{p}}_{(2 \kappa-1) 1}^{ \pm} & \tilde{\mathfrak{p}}_{(2 \kappa-1) 2}^{ \pm} & \ldots & \ldots & \tilde{\mathfrak{p}}_{(2 \kappa-1) h}^{ \pm} & \ldots
\end{array}
$$

we get numberings on $\mathcal{X}_{(2 \kappa-1)}^{+}:=\bigcup_{i=1}^{2 \kappa-1} \mathcal{X}_{i}^{+}$and $\mathcal{X}_{(2 \kappa-1)}^{-}:=\bigcup_{j=1}^{2 \kappa-1} \mathcal{X}_{j}^{-}$:

$$
\tilde{\mathfrak{p}}_{11}^{+}, \tilde{\mathfrak{p}}_{12}^{+}, \tilde{\mathfrak{p}}_{21}^{+}, \tilde{\mathfrak{p}}_{13}^{+}, \tilde{\mathfrak{p}}_{22}^{+}, \tilde{\mathfrak{p}}_{31}^{+}, \ldots \quad \text { and } \quad \tilde{\mathfrak{p}}_{11}^{-}, \tilde{\mathfrak{p}}_{12}^{-}, \tilde{\mathfrak{p}}_{21}^{-}, \tilde{\mathfrak{p}}_{13}^{-}, \tilde{\mathfrak{p}}_{22}^{-}, \tilde{\mathfrak{p}}_{31}^{-}, \ldots
$$

Then, if $\tilde{\mathfrak{p}}_{\ell h}^{-}\left(\right.$or $\left.\tilde{\mathfrak{p}}_{\ell h}^{+}\right)$is the $m$ th member in $\mathcal{X}_{(2 \kappa-1)}^{-}\left(\right.$or in $\left.\mathcal{X}_{(2 \kappa-1)}^{+}\right)$, then we may also assume that $\pi_{j}, p_{i}$ satisfy

$$
\pi_{2 m+1} \in \tilde{\mathfrak{P}}_{\ell h}^{+} \quad \text { and } \quad p_{2 m+1} \in \tilde{\mathfrak{P}}_{\ell h}^{-} \quad \text { for } m+1 \leq \kappa .
$$


5.5. Decomposition of $\rho_{\kappa+1}$ and choice of $\pi_{2 \kappa}, p_{2 \kappa}$

Let $e_{(\kappa+1) 1}, e_{(\kappa+1) 2}, \ldots, e_{(\kappa+1)(2 \kappa-1)}, f_{(\kappa+1) 1}, f_{(\kappa+1) 2}, \ldots, f_{(\kappa+1)(2 \kappa-1)}$ be natural numbers that satisfy the following

$$
\rho_{\kappa+1} \in \bigcap_{i=1}^{2 \kappa-1}\left(p_{i}^{e_{(\kappa+1) i}}, \tilde{\mathfrak{q}}_{2 \kappa-2}^{+}\right) \cap \bigcap_{j=1}^{2 \kappa-1}\left(\pi_{j}^{f_{(\kappa+1) j}}, \tilde{\mathfrak{q}}_{2 \kappa-2}^{-}\right)
$$

and

$$
\rho_{\kappa+1} \notin \bigcup_{i=1}^{2 \kappa-1}\left(p_{i}^{e_{(\kappa+1) i}+1}, \tilde{\mathfrak{q}}_{2 \kappa-2}^{+}\right) \cup \bigcup_{j=1}^{2 \kappa-1}\left(\pi_{j}^{f_{(\kappa+1) j}+1}, \tilde{\mathfrak{q}}_{2 \kappa-2}^{-}\right) .
$$

Then

$$
\rho_{\kappa+1} \equiv p_{1}^{e_{(\kappa+1) 1}} \ldots p_{2 \kappa-1}^{e_{(\kappa+1)(2 \kappa-1)}} \cdot \pi_{1}^{f_{(\kappa+1) 1}} \ldots \pi_{2 \kappa-1}^{f_{(\kappa+1)(2 \kappa-1)}} \cdot \beta_{2 \kappa} \cdot u_{2 \kappa} \quad\left(\bmod \tilde{\mathfrak{q}}_{2 \kappa-1}^{-}\right)
$$

with $\beta_{2 \kappa} \in R_{2 \kappa-1}\left[q_{2 \kappa-1} \alpha_{(2 \kappa-1)(2 \kappa-1)}^{+}\right]$.

Here $\beta_{2 \kappa}$ is either a prime element or a unit in $\tilde{R}_{2 \kappa-1} / \tilde{\mathfrak{q}}_{2 \kappa-1}^{-}$and $u_{2 \kappa}$ is a unit in $\tilde{R}_{2 \kappa-2}$ (cf. Lemma 4.16). If $\beta_{2 \kappa}$ is a prime element, then put $\pi_{2 \kappa}:=\beta_{2 \kappa}$. Then

$$
\pi_{2 \kappa} \notin \bigcup \operatorname{Ass}\left(\tilde{R}_{2 \kappa-1} /\left(q_{2 \kappa-1}, \tilde{\mathfrak{q}}_{2 \kappa-1}\right)\right)
$$

and $\pi_{2 \kappa} \tilde{R}_{2 \kappa-1}+\tilde{\mathfrak{q}}_{2 \kappa-1}^{-}$is a prime ideal different from $p_{i} \tilde{R}_{2 \kappa-1}+\tilde{\mathfrak{q}}_{2 \kappa-1}^{+}$and $\pi_{j} \tilde{R}_{2 \kappa-1}+\tilde{\mathfrak{q}}_{2 \kappa-1}^{-}$for $i, j \leq 2 \kappa-1$. Otherwise, take $\pi_{2 \kappa} \in \mathcal{P} \cap R_{0}$ such that the image of $\pi_{2 \kappa}$ is a prime element in $\tilde{R}_{2 \kappa-1} / \tilde{\mathfrak{q}}_{2 \kappa-1}^{-}$,

$$
\pi_{2 \kappa} \notin \bigcup \operatorname{Ass}\left(\tilde{R}_{2 \kappa-1} /\left(q_{2 \kappa-1}, \tilde{\mathfrak{q}}_{2 \kappa-1}\right)\right),
$$

and $\pi_{2 \kappa} \tilde{R}_{2 \kappa-1}+\tilde{\mathfrak{q}}_{2 \kappa-1}^{-}$is a prime ideal different from $p_{i} \tilde{R}_{2 \kappa-1}+\tilde{\mathfrak{q}}_{2 \kappa-1}^{+}$and $\pi_{j} \tilde{R}_{2 \kappa-1}+\tilde{\mathfrak{q}}_{2 \kappa-1}^{-}$for $i, j \leq 2 \kappa-1$.

In both cases, let $p_{2 \kappa} \in \mathcal{P} \cap R_{2 \kappa-2}$ (or $\mathcal{P} \cap R_{0}$ ) such that the image of $p_{2 \kappa}$ is a prime element in $\tilde{R}_{2 \kappa-1} / \tilde{\mathfrak{q}}_{2 \kappa-1}^{+}$,

$$
p_{2 \kappa} \notin \bigcup \operatorname{Ass}\left(\tilde{R}_{2 \kappa-1} /\left(\theta_{2 \kappa}, \tilde{\mathfrak{q}}_{2 \kappa-1}\right)\right),
$$

and $p_{2 \kappa} \tilde{R}_{2 \kappa-1}+\tilde{\mathfrak{q}}_{2 \kappa-1}^{+}$is a prime ideal different from $p_{i} \tilde{R}_{2 \kappa-1}+\tilde{\mathfrak{q}}_{2 \kappa-1}^{+}$and $\pi_{j} \tilde{R}_{2 \kappa-1}+\tilde{\mathfrak{q}}_{2 \kappa-1}^{-}$for $i+1, j \leq 2 \kappa$. Thus,

$$
\pi_{j} \in R_{j-1}\left[q_{j-1} \alpha_{(j-1)(j-1)}^{+}\right] \quad \text { and } \quad p_{i} \in \mathcal{P} \cap R_{i-2} \quad \text { for } i, j \leq 2 \kappa,
$$

and, for $m \leq 2 \kappa$,

- $q_{m}, \theta_{m}$ form an $\left(\tilde{R}_{m-1} / \tilde{\mathfrak{q}}_{m-1}\right)$-sequence,

- $q_{m}, \theta_{m}$ form an $\left(\tilde{R}_{m} / \tilde{\mathfrak{q}}_{m}\right)$-sequence,

- $p_{1} \tilde{R}_{m-1}+\tilde{\mathfrak{q}}_{m-1}^{+}, \ldots, p_{m} \tilde{R}_{m-1}+\tilde{\mathfrak{q}}_{m-1}^{+}$and $\pi_{1} \tilde{R}_{m-1}+\tilde{\mathfrak{q}}_{m-1}^{-}, \ldots, \pi_{m} \tilde{R}_{m-1}+$ $\tilde{\mathfrak{q}}_{m-1}^{-}$are mutually distinct prime ideals of $\tilde{R}_{m-1}$. 
Now we show that if the $\varepsilon_{m}$ 's are large enough, for $m \leq 2 \kappa$,

$$
\begin{gathered}
q_{m}, z_{1 m m}, \ldots, z_{n m m} \text { form an }\left(R_{m} / \mathfrak{q}_{m}^{\Delta}\right) \text {-sequence, } \\
s_{m m}, z_{1 m m}, \ldots, z_{n m m} \text { form an }\left(R_{m} / \mathfrak{q}_{m}^{\Delta}\right) \text {-sequence. }
\end{gathered}
$$

Proof of (5.5.2) and (5.5.3)

By definition, it is enough to show that, for $m \leq 2 \kappa$,

$q_{m}, z_{1 m m}, \ldots, z_{n m m}, g_{1 m m}^{+}+w_{1 m}, \ldots, g_{r m m}^{+}+w_{r m}$ form an $R_{m}$-sequence,

$s_{m m}, z_{1 m m}, \ldots, z_{n m m}, g_{1 m m}^{+}+w_{1 m}, \ldots, g_{r m m}^{+}+w_{r m}$ form an $R_{m}$-sequence.

By induction, we may assume for $\ell \leq 2 \kappa-1$ that

$$
\begin{aligned}
& q_{\ell}, z_{1 \ell \ell}, \ldots, z_{n \ell \ell}, g_{1 \ell \ell}^{+}+w_{1 \ell}, \ldots, g_{r \ell \ell}^{+}+w_{r \ell} \text { form an } R_{\ell \text {-sequence, }} \\
& s_{\ell \ell}, z_{1 \ell \ell}, \ldots, z_{n \ell \ell}, g_{1 \ell \ell}^{+}+w_{1 \ell}, \ldots, g_{r \ell \ell}^{+}+w_{r \ell} \text { form an } R_{\ell \text {-sequence. }}
\end{aligned}
$$

Because $z_{1 m m}, \ldots, z_{n m m}, g_{1 m m}^{+}+w_{1 m}, \ldots, g_{r m m}^{+}+w_{r m}$ is a subregular system of parameters of $R_{m}, \mathfrak{P}_{m}:=\left(z_{1 m m}, \ldots, z_{n m m}, g_{1 m m}^{+}+w_{1 m}, \ldots, g_{r m m}^{+}+w_{r m}\right)$ is a prime ideal generated by an $R_{m}$-sequence. By construction, we have

$$
\begin{aligned}
s_{m m} & =s_{(m-1)(m-1)} r_{m}+q_{m}^{\varepsilon_{m}^{\prime}} \tau_{m}, \\
z_{i m m} & =x_{i m}+\left(y_{i}+\sum_{h=1}^{m} b_{i h} s_{h m}^{\varepsilon_{h}}\right) \\
& =z_{i(m-1)(m-1)}+q_{m}^{\varepsilon_{m}^{\prime}} \lambda_{i m}+s_{(m-1)(m-1)}^{\varepsilon_{m}} r_{i m}^{*}, \\
z_{i(m-1)(m-1)} & =z_{i(m-1)(m-2)}+b_{i(m-1)} s_{(m-1)(m-1)}^{\varepsilon_{m-1}}, \\
g_{j m m}^{+}+w_{j m} & =g_{j(m-1)(m-1)}^{+}+w_{j(m-1)}+q_{m}^{\varepsilon_{m}^{\prime}} \kappa_{j m},
\end{aligned}
$$

where (cf. (2.5.1), (2.3.6), (2.5.4))

$$
\begin{aligned}
& r_{m} \in R_{m-1}, r_{i m}^{*} \in R_{m-1}\left[\underline{b_{m}}\right], w_{j m} \in R_{m-1}\left[\underline{c_{m}}\right], z_{i m \ell} \in R_{m-1}\left[\underline{a_{m}}, \underline{b_{\ell}}\right], \\
& s_{m m}, \tau_{m}, g_{j m m}^{+}, \kappa_{j m} \in R_{m-1}\left[\underline{a_{m}}, \underline{c_{m}}\right], \text { and } \lambda_{i m} \in R_{m-1}\left[\underline{a_{m}}, \underline{b_{m}}, \underline{c_{m}}\right] .
\end{aligned}
$$

Thus, to get the assertion, it suffices to show that $q_{m}, r_{m} \notin \mathfrak{P}_{m}$ for sufficiently large $\varepsilon_{m}$. Namely,

$$
q_{m}, r_{m} \notin \mathfrak{P}_{m-1} \text {. }
$$

In fact, this results from

$$
\begin{aligned}
\left(z_{(m-1)(m-1)}, \tilde{\mathfrak{q}}_{m-1}\right) \cap R_{m-2} & =\left(z_{(m-1)(m-1)}, \tilde{\mathfrak{q}}_{m-1}^{\Delta}\right) \cap R_{m-2} \\
& =\left(z_{(m-1)(m-1)}, \mathfrak{q}_{m-1}^{\Delta}\right) \cap R_{m-2} \\
& =\mathfrak{P}_{m-1} \cap R_{m-2} \\
& =(0)
\end{aligned}
$$


and from either $q_{m}, \pi_{m} \in R_{m-2}$ or

$$
\rho \equiv p_{1}^{e_{1}} \cdots \pi_{m-1}^{f_{m-1}} \cdot \pi_{m} \cdot u_{m} \quad\left(\bmod \tilde{\mathfrak{q}}_{m-1}^{-}\right)
$$

where $\rho \in R_{m-2}$ with $\pi_{m} \in R_{m-1}\left[q_{m-1} \alpha_{(m-1)(m-1)}^{+}\right]$(cf. (5.4.1), (5.5.1)). This completes the proof of (5.5.2) and (5.5.3).

Moreover, with the notation and assumptions above, we show that

$$
q_{m}, z_{1 m m}, \ldots, z_{n m m} \text { form an }\left(R_{m} / \mathfrak{q}_{m}^{\nabla}\right) \text {-sequence for } m \leq 2 \kappa .
$$

\section{Proof of (5.5.4)}

First of all, by inductive hypothesis, we may assume that

$$
q_{\ell}, z_{1 \ell \ell}, \ldots, z_{n \ell \ell} \text { form an }\left(R_{\ell} / \mathfrak{q}_{\ell}^{\nabla}\right) \text {-sequence for } \ell \leq 2 \kappa-1 \text {. }
$$

By construction, $R_{m} /\left(\pi_{1}, \mathfrak{q}_{m}^{\nabla}\right) \cong\left(R_{m-1} /\left(\pi_{1}, \mathfrak{q}_{m-1}^{\nabla}\right)\right)\left(\underline{a_{m}}, \underline{b_{m}}, \underline{c_{m}}\right)$ (cf. (2.3.8)). Then, because $g_{1 m m}^{-}-w_{1 m}, \ldots, g_{r m m}^{-}-w_{r m}, z_{1(m-1)(m-1)}, \ldots, z_{n(m-1)(m-1)}$ form a subregular system of parameters of $R_{m} / \pi_{1} R_{m}, \overline{\mathfrak{P}}_{m-1}:=\left(\bar{z}_{1(m-1)(m-1)}\right.$, $\left.\ldots, \bar{z}_{n(m-1)(m-1)}\right)$ is a prime ideal generated by an $\left(R_{m-1} /\left(\pi_{1}, \mathfrak{q}_{m-1}^{\nabla}\right)\right)$-sequence. If we denote by $\bar{q}_{m}$ and $\bar{z}_{i(m-1)(m-1)}$ the images of $q_{m}$ and $z_{i(m-1)(m-1)}$ in $R_{m-1} /\left(\pi_{1}, \mathfrak{q}_{m-1}^{\nabla}\right), \bar{z}_{i m m}=\bar{z}_{i(m-1)(m-1)}+a_{i m} \bar{q}_{m}^{\varepsilon_{m}}$ in $\left(R_{m-1} /\left(\pi_{1}, \mathfrak{q}_{m-1}^{\nabla}\right)\right)\left[\underline{a_{m}}, \underline{b_{m}}\right.$, $\left.\underline{c_{m}}\right]$. On the other hand, $p_{j} \in R_{j-2}$ for $j \geq 2$. Hence, we may further assume that

$$
\overline{\mathfrak{P}}_{\ell} \cap\left(R_{\ell-1} /\left(\pi_{1}, \mathfrak{q}_{\ell-1}^{\nabla}\right)\right)=(0) \text { for } \ell \leq 2 \kappa-1 \text {. }
$$

In particular, $\bar{q}_{m} \notin \overline{\mathfrak{P}}_{m-1}$. Thus by Lemma 1.1, $\pi_{1}, q_{m}, z_{1 m m}, \ldots, z_{n m m}$ form an $\left(R_{m} / \mathfrak{q}_{m}^{\nabla}\right)$-sequence, and we get the assertion.

Next choose sufficiently large $\mu_{2 \kappa}, \nu_{2 \kappa}, \varepsilon_{2 \kappa}$ such that

$$
\begin{aligned}
& \mu_{1}<\mu_{2}<\cdots<\mu_{m}<\cdots<\mu_{2 \kappa-1}<\mu_{2 \kappa} \\
& \wedge \wedge \wedge \wedge \wedge \wedge \\
& \nu_{1}<\nu_{2}<\cdots<\nu_{m}<\cdots<\nu_{2 \kappa-1}<\nu_{2 \kappa} \\
& \wedge \wedge \wedge \wedge \wedge \wedge \wedge \\
& \varepsilon_{1}<\varepsilon_{2}<\cdots<\varepsilon_{m}<\cdots<\varepsilon_{2 \kappa-1}<\varepsilon_{2 \kappa}
\end{aligned}
$$

and

$$
q_{2 \kappa}^{\varepsilon_{2 \kappa}} \theta_{2 \kappa} \in R_{2 \kappa-1} \quad \text { and } \quad \nu_{2 \kappa}>N_{2 \kappa}:=\max \left\{e_{h i}+\nu_{2 \kappa-1}, f_{h j}, f_{h(2 h-2)}\right\}
$$

for $i, j \leq 2 h-3$ and $h \leq \kappa+1$ (cf. (4.5.2), (4.14.3), (4.16.2), (5.4.2), (5.5.2), (5.5.3)). Finally, we give enumerations on

$$
\begin{aligned}
& \mathcal{X}_{2 \kappa}^{+}:=\text {the set of height-one prime ideals of } \tilde{R}_{2 \kappa-1}(\boldsymbol{a}, \boldsymbol{b}, \boldsymbol{c}) /\left(p_{2 \kappa}, \tilde{\mathfrak{q}}_{2 \kappa-1}^{+}\right), \\
& \mathcal{X}_{2 \kappa}^{-}:=\text {the set of height-one prime ideals of } \tilde{R}_{2 \kappa-1}(\boldsymbol{a}, \boldsymbol{b}, \boldsymbol{c}) /\left(\pi_{2 \kappa}, \tilde{\mathfrak{q}}_{2 \kappa-1}^{-}\right) .
\end{aligned}
$$

Namely,

$$
\begin{aligned}
& \mathcal{X}_{2 \kappa}^{+}:=\left\{\tilde{\mathfrak{p}}_{(2 \kappa) h}^{+}:=\tilde{\mathfrak{P}}_{(2 \kappa) h}^{+} /\left(p_{2 \kappa}, \tilde{\mathfrak{q}}_{2 \kappa-1}^{+}\right)\right\} \text {such that } \tilde{\mathfrak{P}}_{(2 \kappa) h}^{+} \subset \tilde{R}_{2 \kappa-1}(\boldsymbol{a}, \boldsymbol{b}, \boldsymbol{c})_{\leq h-2}, \\
& \mathcal{X}_{2 \kappa}^{-}:=\left\{\tilde{\mathfrak{p}}_{(2 \kappa) h}^{-}:=\tilde{\mathfrak{P}}_{(2 \kappa) h}^{-} /\left(\pi_{2 \kappa}, \tilde{\mathfrak{q}}_{2 \kappa-1}^{-}\right)\right\} \text {such that } \tilde{\mathfrak{P}}_{(2 \kappa) h}^{-} \subset \tilde{R}_{2 \kappa-1}(\boldsymbol{a}, \boldsymbol{b}, \boldsymbol{c})_{\leq h-2} .
\end{aligned}
$$


5.6. Choice of $\pi_{2 \kappa+1}$ and $p_{2 \kappa+1}$

When $\tilde{\mathfrak{p}}_{\ell h}^{+}:=\tilde{\mathfrak{P}}_{\ell h}^{+} /\left(p_{\ell}, \tilde{\mathfrak{q}}_{\ell-1}^{+}\right) \subset \tilde{R}_{\ell-1}(\boldsymbol{a}, \boldsymbol{b}, \boldsymbol{c}) /\left(p_{\ell}, \tilde{\mathfrak{q}}_{\ell-1}^{+}\right)$is the $\kappa$ th member in $\mathcal{X}_{(2 \kappa)}^{+}=\bigcup_{i=1}^{2 \kappa} \mathcal{X}_{i}^{+}$, take $\pi_{2 \kappa+1}$ as a prime element of $\mathcal{P} \cap R_{2 \kappa-1}$ such that

- $\pi_{2 \kappa+1} \in \tilde{\mathfrak{P}}_{\ell h}^{+}$whose image is a prime element in $\tilde{R}_{2 \kappa} / \tilde{\mathfrak{q}}_{2 \kappa}^{-}$,

- $\pi_{2 \kappa+1} \notin \bigcup \operatorname{Ass}\left(\tilde{R}_{2 \kappa} /\left(q_{2 \kappa}, \tilde{\mathfrak{q}}_{2 \kappa}\right)\right)$,

- $\pi_{2 \kappa+1} \tilde{R}_{2 \kappa}+\tilde{\mathfrak{q}}_{2 \kappa}^{-}$is a prime ideal different from $p_{i} \tilde{R}_{2 \kappa}+\tilde{\mathfrak{q}}_{2 \kappa}^{+}$, and $\pi_{j} \tilde{R}_{2 \kappa}+\tilde{\mathfrak{q}}_{2 \kappa}^{-}$ for $i, j \leq 2 \kappa$.

Next when $\tilde{\mathfrak{p}}_{\ell h}^{-}:=\tilde{\mathfrak{P}}_{\ell h}^{-} /\left(\pi_{\ell}, \tilde{\mathfrak{q}}_{\ell-1}^{-}\right) \subset \tilde{R}_{\ell-1}(\boldsymbol{a}, \boldsymbol{b}, \boldsymbol{c}) /\left(\pi_{\ell}, \tilde{\mathfrak{q}}_{\ell-1}^{-}\right)$is the $\kappa$ th member in $\mathcal{X}_{(2 \kappa)}^{-}=\bigcup_{j=1}^{2 \kappa} \mathcal{X}_{j}^{-}$, choose $p_{2 \kappa+1} \in \mathcal{P} \cap R_{2 \kappa-1}$ that satisfies the following:

- $p_{2 \kappa+1} \in \tilde{\mathfrak{P}}_{\ell h}^{-}$whose image is a prime element in $\tilde{R}_{2 \kappa} / \tilde{\mathfrak{q}}_{2 \kappa}^{+}$,

- $p_{2 \kappa+1} \notin \bigcup \operatorname{Ass}\left(\tilde{R}_{2 \kappa} /\left(\theta_{2 \kappa+1}, \tilde{\mathfrak{q}}_{2 \kappa}\right)\right)$,

- $p_{2 \kappa+1} \tilde{R}_{2 \kappa}+\tilde{\mathfrak{q}}_{2 \kappa}^{+}$is a prime ideal different from $p_{i} \tilde{R}_{2 \kappa}+\tilde{\mathfrak{q}}_{2 \kappa}^{+}$, and $\pi_{j} \tilde{R}_{2 \kappa}+\tilde{\mathfrak{q}}_{2 \kappa}^{-}$ for $i+1, j \leq 2 \kappa+1$.

Hence,

$$
\pi_{j} \in R_{j-1}\left[q_{j-1} \underline{\alpha_{(j-1)(j-1)}^{+}}\right] \quad \text { and } \quad p_{i} \in \mathcal{P} \cap R_{i-2} \quad \text { for } i, j \leq 2 \kappa+1 .
$$

Further, for $m \leq 2 \kappa+1$

- $q_{m}, \theta_{m}$ form an $\left(\tilde{R}_{m-1} / \tilde{\mathfrak{q}}_{m-1}\right)$-sequence,

- $q_{m}, \theta_{m}$ form an $\left(\tilde{R}_{m} / \tilde{\mathfrak{q}}_{m}\right)$-sequence,

- $p_{1} \tilde{R}_{m-1}+\tilde{\mathfrak{q}}_{m-1}^{+}, \ldots, p_{m} \tilde{R}_{m-1}+\tilde{\mathfrak{q}}_{m-1}^{+}$and $\pi_{1} \tilde{R}_{m-1}+\tilde{\mathfrak{q}}_{m-1}^{-}, \ldots, \pi_{m} \tilde{R}_{m-1}+$ $\tilde{\mathfrak{q}}_{m-1}^{-}$are mutually distinct prime ideals of $\tilde{R}_{m-1}$.

Now, by the same arguments as in (5.5.2), (5.5.3), and (5.5.4), we get for $m \leq$ $2 \kappa+1$

$$
q_{m}, z_{1 m m}, \ldots, z_{n m m} \text { form an }\left(R_{m} / \mathfrak{q}_{m}^{\Delta}\right) \text {-sequence, }
$$

and if the $\varepsilon_{m}$ 's are large enough, then

$$
\begin{gathered}
s_{m m} z_{1 m m}, \ldots, z_{n m m} \text { form an }\left(R_{m} / \mathfrak{q}_{m}^{\Delta}\right) \text {-sequence, } \\
q_{m}, z_{1 m m}, \ldots, z_{n m m} \text { form an }\left(R_{m} / \mathfrak{q}_{m}^{\nabla}\right) \text {-sequence. }
\end{gathered}
$$

Moreover, choose sufficiently large $\mu_{2 \kappa+1}, \nu_{2 \kappa+1}, \varepsilon_{2 \kappa+1}$ such that

$$
\begin{aligned}
& \mu_{1}<\mu_{2}<\cdots<\mu_{m}<\cdots<\mu_{2 \kappa}<\mu_{2 \kappa+1} \\
& \wedge \wedge \wedge \wedge \wedge \wedge \\
& \nu_{1}<\nu_{2}<\cdots<\nu_{m}<\cdots<\nu_{2 \kappa}<\nu_{2 \kappa+1} \\
& \wedge \wedge \wedge \wedge \wedge \wedge \\
& \varepsilon_{1}<\varepsilon_{2}<\cdots<\varepsilon_{m}<\cdots<\varepsilon_{2 \kappa}<\varepsilon_{2 \kappa+1}
\end{aligned}
$$

and $q_{2 \kappa+1}^{\varepsilon_{2 \kappa+1}} \theta_{2 \kappa+1} \in R_{2 \kappa}$ (cf. (4.5.2), (4.14.3), (4.16.2), (5.4.2), (5.5.5), (5.6.2), $(5.6 .3))$. 
Finally, we give enumerations on

$\mathcal{X}_{2 \kappa+1}^{+}:=$the set of height-one prime ideals of $\tilde{R}_{2 \kappa}(\boldsymbol{a}, \boldsymbol{b}, \boldsymbol{c}) /\left(p_{2 \kappa+1}, \tilde{\mathfrak{q}}_{2 \kappa}^{+}\right)$,

$\mathcal{X}_{2 \kappa+1}^{-}:=$the set of height-one prime ideals of $\tilde{R}_{2 \kappa}(\boldsymbol{a}, \boldsymbol{b}, \boldsymbol{c}) /\left(\pi_{2 \kappa+1}, \tilde{\mathfrak{q}}_{2 \kappa}^{-}\right)$.

Namely,

$\mathcal{X}_{2 \kappa+1}^{+}:=\left\{\tilde{\mathfrak{p}}_{(2 \kappa+1) h}^{+}:=\tilde{\mathfrak{P}}_{(2 \kappa+1) h}^{+} /\left(p_{2 \kappa+1}, \tilde{\mathfrak{q}}_{2 \kappa}^{+}\right)\right\}$with $\tilde{\mathfrak{P}}_{(2 \kappa+1) h}^{+} \subset \tilde{R}_{2 \kappa}(\boldsymbol{a}, \boldsymbol{b}, \boldsymbol{c})_{\leq h-2}$,

$\mathcal{X}_{2 \kappa+1}^{-}:=\left\{\tilde{\mathfrak{p}}_{(2 \kappa+1) h}^{-}:=\tilde{\mathfrak{P}}_{(2 \kappa+1) h}^{-} /\left(\pi_{2 \kappa+1}, \tilde{\mathfrak{q}}_{2 \kappa}^{-}\right)\right\}$with $\tilde{\mathfrak{P}}_{(2 \kappa+1) h}^{-} \subset \tilde{R}_{2 \kappa}(\boldsymbol{a}, \boldsymbol{b}, \boldsymbol{c})_{\leq h-2}$.

This completes the proof of Proposition 5.2.

\section{Construction}

\subsection{Notation and terminology}

With the notation, assumptions, and numberings as in the previous sections, let

$$
\begin{aligned}
B_{k} & :=\tilde{R}_{k}\left[\underline{\alpha_{k k}^{-}}, \underline{\beta_{k k}^{+}}, \underline{\beta_{k k}^{-}}\right], \quad \mathfrak{M}_{k}:=\left(z_{0}, \underline{z}, \underline{w}, \underline{\alpha_{k k}^{+}}, \underline{\alpha_{k k}^{-}}, \underline{\beta_{k k}^{+}}, \underline{\beta_{k k}^{-}}\right) B_{k}, \\
B & :=\bigcup B_{k}, \quad \text { and } \quad M:=\bigcup \mathfrak{M}_{k}=\left(z_{0}, \underline{z}, \underline{w}\right) B,
\end{aligned}
$$

where $\underline{w}:=\left(w_{1}, \ldots, w_{r}\right), \underline{z}:=\left(z_{1}, \ldots, z_{n}\right), \alpha_{k k}^{+}:=\left(\alpha_{1 k k}^{+}, \ldots, \alpha_{r k k}^{+}\right), \alpha_{k k}^{-}:=\left(\alpha_{1 k k}^{-}\right.$, $\left.\ldots, \alpha_{r k k}^{-}\right), \beta_{k k}^{+}:=\left(\beta_{1 k k}^{+}, \ldots, \beta_{s k k}^{+}\right), \beta_{k k}^{-}:=\left(\overline{\beta_{1 k k}^{-}}, \ldots, \beta_{s k k}^{-}\right)$, and $k=\overline{1,2}, \ldots$ Then we have the following result.

\section{LEMMA 6.2}

With the notation above, $M$ is a maximal ideal of $B$. And we have the canonical isomorphism $R / \mathfrak{n} \stackrel{\cong}{\longrightarrow} B / M$.

\section{Proof}

By definitions and by relations among $\alpha_{j k k}^{+}, \alpha_{j k k}^{-}, \beta_{h k k}^{+}$, and $\beta_{h k k}^{-}$, it is clear that all these elements are in $M$ (cf. (2.4.2), (3.2.1), (3.2.2)). To get the assertion, it remains to verify that $M \neq B$.

Assume the contrary. That is, $M=B$. Then there exist $\gamma_{0}, \gamma_{1}, \ldots, \gamma_{n}, \delta_{1}, \ldots$, $\delta_{r} \in B$ that satisfy

$$
\gamma_{0} \cdot z_{0}+\gamma_{1} \cdot z_{1}+\cdots+\gamma_{n} \cdot z_{n}+\delta_{1} \cdot w_{1}+\cdots+\delta_{r} \cdot w_{r}=1
$$

We may assume that $\gamma_{i}, \delta_{j} \in B_{k}$ for sufficiently large $k$. Thus there exist $c_{i}$, $d_{j} \in \tilde{R}_{k}$ and $\nu \in \mathbf{N}$ such that

$$
q_{k}^{\nu} \cdot \theta_{k}^{\nu}\left(\gamma_{i}-c_{i}\right), q_{k}^{\nu} \cdot \theta_{k}^{\nu}\left(\delta_{j}-d_{j}\right) \in \tilde{\mathfrak{q}}_{k} \quad(\text { cf. (2.3.10), (3.2.1), (3.2.2)). }
$$

Hence,

$$
q_{k}^{\nu} \cdot \theta_{k}^{\nu}\left(c_{0} \cdot z_{0}+c_{1} \cdot z_{1}+\cdots+c_{n} \cdot z_{n}+d_{1} \cdot w_{1}+\cdots+d_{r} \cdot w_{r}-1\right) \in \tilde{\mathfrak{q}}_{k}
$$

and therefore $q_{k}^{\nu} \cdot \theta_{k}^{\nu} \in \tilde{\mathfrak{q}}_{k}$. This contradicts the choice of $p_{\ell}$ and $\pi_{m}$ (cf. [8, Lemma 1.4], (5.2.2)). 


\section{LEMMA 6.3}

With the notation above, $p_{i}$ and $\pi_{j}$ are prime elements of $B$ that satisfy the following:

$$
\begin{array}{r}
\bigcup_{k}\left(\tilde{R}_{i}(\boldsymbol{a}, \boldsymbol{b}, \boldsymbol{c}) /\left(p_{i}, \tilde{\mathfrak{q}}_{i}^{+}\right)\right)\left[\underline{\bar{\alpha}_{k k}^{-}}\right] \stackrel{\cong}{\longrightarrow} B / p_{i} B, \\
\bigcup_{k}\left(\left(\tilde{R}_{j}(\boldsymbol{a}, \boldsymbol{b}, \boldsymbol{c}) /\left(\pi_{j}, \tilde{\mathfrak{q}}_{j}^{-}\right)\right)\left[\underline{\bar{\alpha}_{k k}^{+}}\right]\right)_{U_{j k}} \stackrel{\cong}{\longrightarrow} B / \pi_{j} B,
\end{array}
$$

where $\bar{\alpha}_{k k}^{-}$is the image of $\underline{\alpha_{k k}^{-}}$in the field of quotients of $\tilde{R}_{i}(\boldsymbol{a}, \boldsymbol{b}, \boldsymbol{c}) /\left(p_{i}, \tilde{\mathfrak{q}}_{i}^{+}\right)$ and $\bar{\alpha}_{k k}^{+}$is the image of $\alpha_{k k}^{+} \overline{\text { in }}$ the field of quotients of $\tilde{R}_{j}(\boldsymbol{a}, \boldsymbol{b}, \boldsymbol{c}) /\left(\pi_{j}, \tilde{\mathfrak{q}}_{j}^{-}\right)$with $U_{j k}:=1+\left(\tilde{\mathfrak{n}}_{j}, \alpha_{k k}^{+}\right)$, respectively.

\section{Proof}

First of all, putting $\tilde{R}:=\bigcup_{k} \tilde{R}_{k}$, we note that $\tilde{R}_{k}(\boldsymbol{a}, \boldsymbol{b}, \boldsymbol{c}) /\left(p_{i}, \tilde{\mathfrak{q}}_{k}^{+}\right) \cong \tilde{R} / p_{i} \tilde{R}$ for every $k \geq i$. In fact, this results from the following commutative diagram:

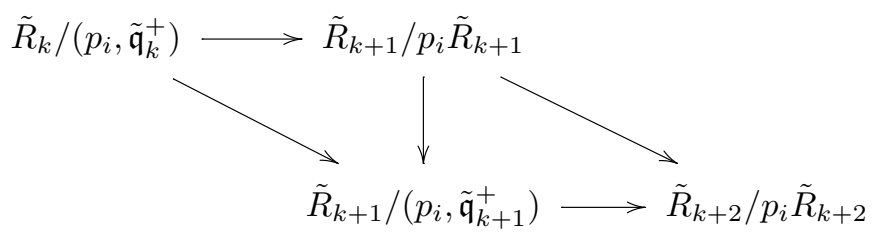

where the left diagonal map is injective (cf. (4.5.2)). This also shows that $p_{i} \tilde{R}_{k+1} \cap$ $\tilde{R}_{k}=\left(p_{i}, \tilde{\mathfrak{q}}_{k}^{+}\right) \tilde{R}_{k}$ for every $k \geq i$.

Proof of (6.3.1)

By the remark just above, we get the following commutative diagram, because $\tilde{R}_{k}\left[1 / \theta_{k+1}\right]=B_{k}\left[1 / \theta_{k+1}\right]$ (cf. (3.2.1), (3.2.2), (5.2.8)):

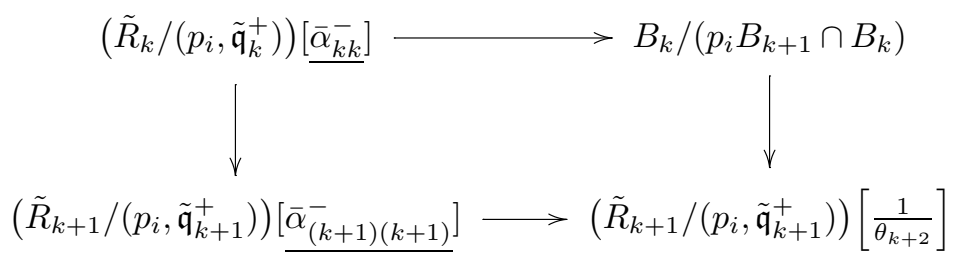

where the upper horizontal map is surjective (cf. (2.4.2), (3.3.1), (3.3.2)) and the left vertical map and the lower horizontal map are injective (cf. (5.2.3), $(5.2 .1))$.

Proof of (6.3.2)

By construction, we get the following commutative diagram, because $\tilde{R}_{k}\left[\alpha_{k k}^{-}\right][1 /$ $\left.q_{k}\right]=B_{k}\left[1 / q_{k}\right]$ (cf. (3.2.1), (3.2.2), (5.2.8)): 


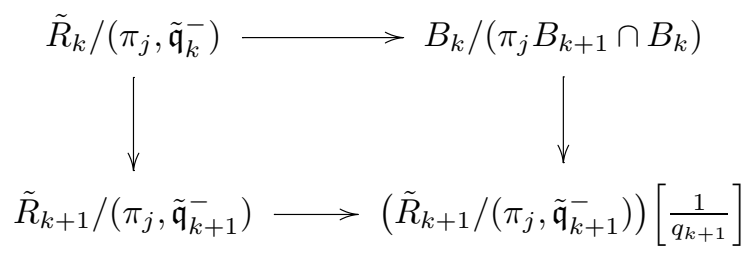

where the upper horizontal map is surjective (cf. (2.4.2), (3.3.1), (3.3.2)) and the left vertical map and the lower horizontal map are injective (cf. (4.14.3), (5.2.3), (5.2.1)).

\section{LEMMA 6.4}

With the notation above, let $A=B_{M}$, and let $\mathfrak{m}=M A$. Then $(A, \mathfrak{m})$ is a Noetherian unique factorization domain.

\section{Proof}

Let $\mathfrak{p}$ be a non-zero prime ideal of $A$. Because $A$ and $R$ have the common field of quotients, $\mathfrak{p} \cap R$ contains a prime element of $R$, say, $\rho$. We may assume that $\rho=\rho_{k+1} \in \mathcal{P}$. Then

$$
\begin{aligned}
\rho= & p_{1}^{e_{1}} \cdots p_{2 k-1}^{e_{2 k-1}} \cdot \pi_{1}^{f_{1}} \cdots \pi_{2 k}^{f_{2 k}} \\
& \times\left[b+\frac{\theta_{2 k}^{\varepsilon_{2 k}}}{\pi_{1}^{f_{1}} \cdots \pi_{2 k}^{f_{2 k}}} \kappa_{2 k-1}^{-}+\frac{q_{2 k}^{\nu_{2 k}}}{p_{1}^{e_{1}} \cdots p_{2 k-1}^{e_{2 k-1}} \cdot q_{2 k-1}^{\nu_{2 k-1}}} \sum d_{j} \alpha_{j 2 k 2 k}^{+}\right. \\
& \left.\quad+\frac{q_{2 k}^{\varepsilon_{2 k}}}{p_{1}^{e_{1}} \cdots p_{2 k-1}^{e_{2 k-1}} \cdot q_{2 k-1}^{\nu_{2 k-1}}} \kappa_{2 k-1}^{+}+\frac{q_{2 k}^{\nu_{2 k}} \theta_{2 k}^{\varepsilon_{2 k}}}{p_{1}^{e_{1}} \cdots p_{2 k-1}^{e_{2 k-1}} \pi_{1}^{f_{1}} \cdots \pi_{2 k}^{f_{2 k}} \cdot q_{2 k-1}^{\nu_{2 k-1}}} \lambda_{2 k-1}^{+}\right] \\
& +p_{1}^{e_{1}} \cdots p_{2 k-1}^{e_{2 k-1}} \sum c_{j}\left(\phi_{j 2 k 2 k}^{-}-w_{j 2 k}\right) \\
& +\frac{q_{2 k}^{\nu_{2 k}}}{q_{2 k-1}^{\nu_{2 k-1}}} \sum e_{j}\left(\phi_{j 2 k 2 k}^{-}-w_{j 2 k}\right)+\left(\frac{q_{2 k}^{\nu_{2 k}}}{q_{2 k-1}^{\nu_{2 k-1}}}\right)^{g_{h}} \sum e_{h}^{\prime} G_{h}\left(\underline{\zeta_{2 k 2 k}}, \underline{\alpha_{2 k 2 k}^{+}}\right),
\end{aligned}
$$

where $b$ is a unit element in $\tilde{R}_{2 k-1}$ (cf. (4.16.5), Lemma 4.17, (5.2.7)). Hence,

$$
\rho=p_{1}^{e_{1}} \cdots p_{2 k-1}^{e_{2 k-1}} \pi_{1}^{f_{1}} \cdots \pi_{2 k}^{f_{2 k}}\left(b+\theta_{2 k} \varepsilon^{\prime}+q_{2 k} \delta^{\prime}+\sum c_{j}^{\prime} \alpha_{j 2 k 2 k}^{-}+\sum d_{h}^{\prime} \beta_{h 2 k 2 k}^{+}\right),
$$

where $\varepsilon^{\prime}, \delta^{\prime}, c_{j}^{\prime}$, and $d_{h}^{\prime} \in \tilde{R}_{2 k-1}\left(\underline{a_{2 k}}, \underline{b_{2 k}}, \underline{c_{2 k}}\right)$. Thus,

$$
\rho A=p_{1}^{e_{1}} \cdots p_{2 k-1}^{e_{2 k-1}} \pi_{1}^{f_{1}} \cdots \pi_{2 k}^{f_{2 k}} A .
$$

That is, $\mathfrak{p}$ contains either $p_{i}$ for some $i \leq 2 k-1$ or $\pi_{j}$ for some $j \leq 2 k$. Because both $A / p_{i} A$ and $A / \pi_{j} A$ are Noetherian domains (cf. (6.3.1), (6.3.2), (5.2.9)), $\mathfrak{p}$ is finitely generated. Therefore, $A$ is Noetherian. Moreover, the argument above shows that if, in addition, $\mathfrak{p}$ is a height-one prime ideal, then $\mathfrak{p}$ is principal (cf. [8, Proposition 5.6]). 


\subsection{Notation}

With the same notation as in previous sections, let $\widehat{R}:=K\left[\left[z_{0}, z_{1}, \ldots, z_{n}, w_{1}, \ldots\right.\right.$, $\left.w_{r}\right]$ ]. Take elements of $\widehat{R}$ :

$$
\begin{aligned}
& \xi_{i}:=x_{i}+a_{i 1} q_{1}^{\varepsilon_{1}}+\cdots+a_{i k} q_{k}^{\varepsilon_{k}}+\cdots=x_{i}+\sum_{k=1}^{\infty} a_{i k} q_{k}^{\varepsilon_{k}}, \\
& \omega_{j}:=w_{j}+c_{j 1} t_{1}^{\varepsilon_{1}}+\cdots+c_{j k} t_{k}^{\varepsilon_{k}}+\cdots=w_{j}+\sum_{k=1}^{\infty} c_{j k} t_{k}^{\varepsilon_{k}} .
\end{aligned}
$$

Let $\varphi_{j}^{+}:=\lim _{k \rightarrow \infty} \phi_{j k k}^{+}, \psi_{j}^{+}:=\varphi_{j}^{+}+\omega_{j}$, and let

$$
\tilde{\alpha}_{j k k}^{+}:=\alpha_{j k k}^{+}-\frac{\psi_{j}^{+}}{q_{k}^{\nu_{k}}}=\frac{1}{q_{k}^{\nu_{k}}}\left(\phi_{j k k}^{+}-\sum_{h>k}^{\infty} c_{j h} t_{h}^{\varepsilon_{h}}-\varphi_{j}^{+}\right) .
$$

By letting $\tilde{\theta}_{k}:=\tilde{\pi}_{1} \cdots \tilde{\pi}_{k}$ with

$$
\tilde{\pi}_{k}:=r_{k}+\sum r_{k H}\left(q_{k-1} \tilde{\alpha}_{(k-1)(k-1)}^{+}\right)^{H} \in \widehat{R} \quad(\text { cf. }(2.3 .12)),
$$

we put

$$
\begin{aligned}
\tilde{\eta}_{i m} & :=y_{i}+b_{i 1} \tilde{\theta}_{1}^{\varepsilon_{1}}+\cdots+b_{i m} \tilde{\theta}_{m}^{\varepsilon_{m}}, \\
\tilde{\eta}_{i} & :=y_{i}+\sum_{k=1}^{\infty} b_{i k} \tilde{\theta}_{k}^{\varepsilon_{k}}, \\
\tilde{\zeta}_{i \ell m} & :=x_{i \ell}+\tilde{\eta}_{i m},
\end{aligned}
$$

and

$$
\tilde{\zeta}_{i}:=\xi_{i}+\tilde{\eta}_{i}
$$

Further, let

$$
\begin{aligned}
\tilde{\varphi}_{j k k} & :=F_{j}\left(\underline{\tilde{\zeta}_{k k}}\right) \\
& =F_{j}^{+}\left(\underline{\tilde{\zeta}_{k k}}\right)+F_{j}^{-}\left(\underline{\tilde{\zeta}_{k k}}\right)=: \tilde{f}_{j k k}^{+}+\tilde{f}_{j k k}^{-}, \\
\tilde{\varphi}_{j} & :=F_{j}(\underline{\tilde{\zeta}}) \\
& =\lim _{k \rightarrow \infty} \tilde{f}_{j k k}^{+}+\lim _{k \rightarrow \infty} \tilde{f}_{j k k}^{-}=: \tilde{\varphi}_{j}^{+}+\tilde{\varphi}_{j}^{-}
\end{aligned}
$$

and

$$
\tilde{\psi}_{j}^{-}:=\tilde{\varphi}_{j}^{-}-\omega_{j}
$$

\section{LEMMA 6.6}

With the notation above, we get

$$
K\left[\left[z_{0}, \tilde{\zeta}_{1}, \ldots, \tilde{\zeta}_{n}\right]\right] /\left(\tilde{\varphi}_{1}, \ldots, \tilde{\varphi}_{r}\right) \cong \widehat{R} /\left(\psi_{1}^{+}, \ldots, \psi_{r}^{+}, \tilde{\psi}_{1}^{-}, \ldots, \tilde{\psi}_{r}^{-}\right) \cong \widehat{A} .
$$


Proof

By Lemma 6.2, we have a canonical surjection $\hat{\iota}: \widehat{R} \rightarrow \widehat{A}$ that gives the following commutative diagram

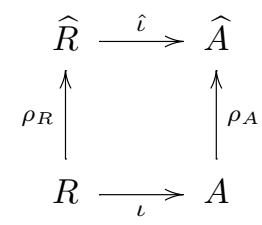

with canonical injections $\iota, \rho_{R}$, and $\rho_{A}$. Thus, we are only to determine Ker $\hat{\iota}$. By definition, $\psi_{j}^{+}-\left(\phi_{j k k}^{+}+w_{j k}\right) \in q_{k+1}^{\nu_{k+1}} \widehat{R}$ and $\phi_{j k k}^{+}+w_{j k}=q_{k}^{\nu_{k}} \alpha_{j k k}^{+} \in q_{k}^{\nu_{k}} A$. Hence, $\hat{\iota}\left(\psi_{j}^{+}\right) \in q_{k}^{\nu_{k}} \widehat{A}$ for every $k \in \mathbf{N}$. Namely, $\psi_{j}^{+} \in \operatorname{Ker} \hat{\iota}$.

On the other hand, $\alpha_{j k k}^{+}=\tilde{\alpha}_{j k k}^{+}$in $\widehat{A}$, because $q_{k}^{\nu_{k}}\left(\alpha_{j k k}^{+}-\tilde{\alpha}_{j k k}^{+}\right)=\psi_{j}^{+} \in \widehat{R}$ and because $q_{k}$ is a non-zero divisor for $\widehat{A}$. Thus, $\zeta_{i}=\tilde{\zeta}_{i}, \phi_{j k k}^{-}=\tilde{f}_{j k k}^{-}, \tilde{\varphi}_{j}^{-}=$ $\lim _{k \rightarrow \infty} \phi_{j k k}^{-}$, and $\tilde{\varphi}_{j}=F_{j}(\zeta)$ in $\widehat{A}$. Moreover, $\tilde{\psi}_{j}^{-}-\left(\tilde{f}_{j k k}^{-}-w_{j k}\right) \in\left(q_{k+1}^{\nu_{k+1}}, \tilde{\pi}_{k+1}^{\nu_{k+1}}\right) \widehat{R}$ and $\hat{\imath}\left(\tilde{f}_{j k k}^{-}-w_{j k}\right)=\phi_{j k k}^{-}-w_{j k}=\theta_{k}^{\nu_{k}} \alpha_{j k k}^{-} \in \theta_{k}^{\nu_{k}} \widehat{A}$. Hence, $\hat{\iota}\left(\tilde{\psi}_{j}^{-}\right) \in\left(q_{k+1}^{\nu_{k+1}}, \pi_{k}^{\nu_{k}}\right) \widehat{A}$ for every $k \in \mathbf{N}$. That is, $\tilde{\psi}_{j}^{-} \in \operatorname{Ker} \hat{\iota}$.

Finally, $\widehat{A} / \pi_{1} \widehat{A} \cong \widehat{R} /\left(\pi_{1}, \mathfrak{q}_{0}^{-}, \psi^{+}\right)$and $\left(\pi_{1}, \mathfrak{q}_{0}^{-}, \psi^{+}\right) \widehat{R}=\left(\pi_{1}, \tilde{\psi}^{-}, \psi^{+}\right) \widehat{R}$, because

$$
A / \pi_{1} A \cong \bigcup_{k}\left(\left(\tilde{R}_{1}(\boldsymbol{a}, \boldsymbol{b}, \boldsymbol{c}) /\left(\pi_{1}, \tilde{\mathfrak{q}}_{1}^{-}\right)\right)\left[\underline{\left.\bar{\alpha}_{k k}^{+}\right]}\right)_{U_{1 k}} \cong\left(\bigcup_{k}\left(R /\left(\pi_{1}, \mathfrak{q}_{0}^{-}\right)\right)\left[\underline{\bar{\alpha}_{k k}^{+}}\right]\right)_{U}\right.
$$

with $U=1+\left(\mathfrak{n}, \underline{\bar{\alpha}_{k k}^{+}}\right)($cf. $(6.3 .2),(4.8 .1))$. Therefore, Ker $\hat{\imath}=\left(\psi_{1}^{+}, \ldots, \psi_{r}^{+}, \tilde{\psi}_{1}^{-}, \ldots\right.$, $\left.\tilde{\psi}_{r}^{-}\right)$. This completes the proof of Theorem 0.1 .

Acknowledgments. The author would like to thank the referees for checking the manuscript carefully and providing useful comments.

\section{References}

[1] M. Brodmann and C. Rotthaus, Local domains with bad sets of formal prime divisors, J. Algebra 75 (1982), 386-394. MR 0653898.

DOI 10.1016/0021-8693(82)90046-1.

[2] A. Grothendieck, Éléments de géométrie algébrique, IV: Étude locale des schémas et des morphismes de schémas, I, Publ. Math. Inst. Hautes Études Sci. 20 (1964); 24 (1965). MR 173675. MR 0199181.

[3] R. C. Heitmann, A noncatenary, normal, local domain, Rocky Mountain J. Math. 12 (1982), 145-148. MR 0649747. DOI 10.1216/RMJ-1982-12-1-145.

[4] S. Loepp, C. Rotthaus, and S. Sword, A class of local Noetherian domains, J. Commut. Algebra 1 (2009), 647-678. MR 2575836. DOI 10.1216/JCA-2009-1-4-647.

[5] H. Matsumura, Commutative Algebra, 2nd ed., Benjamin, New York, 1980. MR 0266911. 
[6] M. Nagata, Local Rings, corrected reprint, Krieger, Huntington, N.Y., 1975. MR 0460307.

[7] J. Nishimura, On ideal-adic completion of Noetherian rings, Kyoto J. Math. 21 (1981), 153-169. MR 0606317.

[8] _ A few examples of local rings I, Kyoto J. Math. 52 (2012), 51-87. MR 2892767. DOI 10.1215/21562261-1503754.

[9] _ A few examples of local rings, III, in preparation.

[10] T. Ogoma, Noncatenary pseudogeometric normal rings, Jpn. J. Math. 6 (1980), 147-163. MR 0615018. DOI 10.4099/math1924.6.147.

[11] Cohen Macaulay factorial domain is not necessarily Gorenstein, Mem. Fac. Sci. Kôchi Univ. Ser. A Math. 3 (1982), 65-74. MR 0643928.

[12] C. Rotthaus, Universell japanische Ringe mit nicht offenem regulärem Ort, Nagoya Math. J. 74 (1979), 123-135. MR 0535964.

Department of Engineering Science, Faculty of Engineering, Osaka Electro-Communication University, Neyagawa, Osaka, Japan;

nisimura@isc.osakac.ac.jp 